\title{
Open Gromov-Witten Invariants on Elliptic K3 Surfaces and Wall-Crossing
}

\section{Citation}

Lin, Yu-Shen. 2013. Open Gromov-Witten Invariants on Elliptic K3 Surfaces and Wall-Crossing. Doctoral dissertation, Harvard University.

\section{Permanent link}

http://nrs.harvard.edu/urn-3:HUL.InstRepos:11158239

\section{Terms of Use}

This article was downloaded from Harvard University's DASH repository, and is made available under the terms and conditions applicable to Other Posted Material, as set forth at http:// nrs.harvard.edu/urn-3:HUL.InstRepos:dash.current.terms-of-use\#LAA

\section{Share Your Story}

The Harvard community has made this article openly available.

Please share how this access benefits you. Submit a story.

\section{Accessibility}


Open Gromov-Witten Invariants on Elliptic K3 Surfaces and Wall-Crossing

A dissertation presented

by

Yu-Shen Lin

to

The Department of Mathematics

in partial fulfillment of the requirements

for the degree of

Doctor of Philosophy

in the subject of

Mathematics

Harvard University

Cambridge, Massachusetts

April 2013 
(c) $2013-\mathrm{Yu}-\mathrm{Shen} \mathrm{Lin}$

All rights reserved. 
Open Gromov-Witten Invariants on Elliptic K3 Surfaces and Wall-Crossing

\begin{abstract}
We defined a new type of open Gromov-Witten invariants $\tilde{\Omega}^{\text {Floer }}$ on hyperKäher manifolds with holomorphic Lagrangian fibration (not necessary compact). Using this new invariant, we prove a version of correspondence theorem between holomorphic discs give rise to non-trivial invariants and tropical discs. Moreover, we prove the above two invariants are the same in an local model and provide an non-trivial example of wall-crossing phenomenon of the open Gromov-Witten invariants on K3 surfaces. We also connect the invariants $\tilde{\Omega}^{\text {Floer }}$ with discs counting on Calabi-Yau 3-folds with K3 fibration via an real analogue of Noether-Lefschetz theory.
\end{abstract}




\section{Contents}

1 Introduction 1

2 Review of Twistorial Construction 4

2.1 Settings and HyperKähler Rotation . . . . . . . . . . . . . . 4

2.2 BPS Counting and Wall-Crossing Formula . . . . . . . 8

2.3 Solving the Riemann-Hilbert Problem . . . . . . . . . . 11

2.4 Local example: Ooguri-Vafa metric . . . . . . . . . . . . . . 14

2.5 Application to Elliptic K3 Surfaces . . . . . . . . . . . 16

3 Scattering Diagrams and Generalized Donaldson-Thomas $\begin{array}{ll}\text { Invariants on K3 Surfaces } & 19\end{array}$

3.1 Construction of Scattering Diagrams on Elliptic K3 Surfaces . 19

3.2 Generalized Donaldson-Thomas Invariants $\Omega^{\text {trop }} \ldots \ldots \ldots$

4 Tropical Geometry on K3 Surfaces 28

4.1 Tropical Discs . . . . . . . . . . . . . . . . . . 28

4.2 Tropical Discs Counting, $\Omega^{\text {trop }}$ and Wall-Crossing . . . . . 30

5 Tropical versus Holomorphic $\quad 34$

5.1 Disc Contribution from Local . . . . . . . . . . . . 38

$5.2 J$-Holomoprhic Discs in the $S^{1}$-Family . . . . . . . . 45

5.3 Kuranishi Structure for Moduli Space of Holomorphic Discs in $S^{1}$-Family $\ldots \ldots \ldots \ldots \ldots \ldots \ldots$

5.4 Orientation ...................... 60

5.5 Reduced $A_{\infty}$ Structure and Floer Theoretic Counting . . . . 61 
5.6 Multiple Cover Formula . . . . . . . . . . . . . 76

5.7 Corresponding Theorem . . . . . . . . . . . . . . 80

5.8 Other Local Models . . . . . . . . . . . . . . . . . . . . 84

6 Quantum Corrected SYZ Transform on the

$\begin{array}{ll}\text { Ooguri-Vafa Space } & 86\end{array}$

7 Real Noether-Lefschetz Theory and Open GW Invariants $\begin{array}{ll}\text { on Calabi-Yau 3-Folds } & 89\end{array}$

7.1 Real Noether-Lefschetz Theory . . . . . . . . . . . . 90

7.2 Open Gromov-Witten Invariants on Calabi-Yau 3-Folds with K3 Fibration . . . . . . . . . . . . . . . . . 92

7.3 Comparison of the Invariants for K3 and Calabi-Yau 3-Folds 94

A Miscellaneous of Kuranishi Structures 100

A.1 Kuranishi Structure and Good Coordinate . . . . . . . . . 100

A.2 Partition of Unity . . . . . . . . . . . . . . . 103

A.3 Multi-Sections and Compatible Perturbations . . . . . . . . 104

A.4 Smooth Correspondence . . . . . . . . . . . . . 109 


\section{Acknowledgements}

Foremost, I want devote my deepest gratitude to my thesis advisor Professor Shing-Tung Yau for constant encouragement and instruction. I benefited much from his insightful viewpoints toward different branches in mathematics and interactions. His seminar broadens my view and later on play an essential role when I developing my thesis.

I am indebted to Professor Kenji Fukaya, Professor Kaoru Ono, Professor Andy Neitzke and Wenxuan Lu for many email correspondences explaining the details of their work. I would like to grateful to Professor Chiu-Chu Melissa Liu for useful discussion and teaching me the localization techniques.

Back to the life to choose mathematics as career, I want to thank my high school teacher Jung-Jeng Tsai and Yi-Xiong Su for brought me to the door of mathematics. I would like to thank Professor Mong-Na Lo Huang and Chun-Kong Law and the special mathematics classes held by National Sun Yat-Sen University for high school students. They help me not just restrict my knowledge in high school mathematics competition but touch some more advance mathematics. I want to thank my teachers in National Taiwan University, especially Professor Jin-Tzu Chen, Professor Ping-Zen Ong, Professor Jung-Kai Chen, Professor I-Hsun Tsai. Professor Jin-Tzu Chen provided three years of analysis course and I luckily gain the knowledge of algebraic topology and Lie group, which is not that popular in NTU at the monent, from Professor Ping-Zen Ong. I started to learn algebraic geometry from Professor Jung-Kai Chen and it is Professor I-Hsun Tsai who brought me to the field of mirror symmetry. Except people from my academic life, 
I would like to thank many friends in "Small Circle" who accompanied me the best four years in college.

Probably I have to thank the doctor who agree that I am seriously nearsighted and only had to serve in the army in twelve days. Therefore, I had a chance to be the research assistant of Professor Chin-Lung Wang during the year spent in National Center of Theoretical Sciences.

Definitely I am grateful to all the Yau's seminar partners including Tosatti Valentino, Chen-Yu Chi, Si Li, Wenxuan Lu, Ming-Tao Chuan, PoNing Chen, Ruifang Song, Yi Li, Jie Zhou, Peter Smillie, Teng Fei, Chung-Jun Tsai, Chien-Hao Liu, Li-Sheng Tseng, Baosen Wu, Yih Sung, Siu-Cheong Lau, Yi Xie, Gang Liu, Yi Zhang, Hao Xu, Ke Zhu, Zhang Peng. I learn different branches of mathematics and benefit much from the seminar. It turns out many things what I talked and I heard from the seminar play important roles in my thesis. Especially, I want to thank Si Li for teaching me so much physics without murmur. He influence much on my taste of mathematics. Also special thanks to Chen-Yu Chi for unlimited "office hour" answering my dumb questions. This unlimited office hour method helps me get over the language barrier in teaching and won two Bok Center Teaching Awards. Another special thanks to Dongning Wang, who spent three hours discussing with me during the last day of a conference in Kansas State University.

At the end, I want to express my superexcellent gratitude to my parents Mr. Horng-Rong Lin and Mrs. Chun-Shu Cheng. Without their painstaking efforts to nurture and raise me up, I won't have any chance to be who I am. Just became a father, I start to realize how willful I am and can't express 
all my gratitude to them within any words. This thesis would also devoted to our new family member, my son Teng-Yuan Li, who make my life more complete. The project became mature around the time we start to expect his coming. Last but not least to my love, my wife Jun-Rong Wei, whom I appreciate her accompany during my hardest period and the sacrifice she made. With her full moral support and taking care of our son, I can write up my thesis without trouble back at home. I would like to take this chance to say I love you. 


\section{Introduction}

The well-known Calabi conjecture solved by Yau guarantees that given a compact Calabi-Yau manifold, there exists a unique Ricci-flat Kähler metric in each prescribed Kähler class [38] in 1978. After existence, the next interesting question to ask is the explicit expression of the metric. [36] suggested that Calabi-Yau manifolds will admit special Lagrangian fibration around large complex limits and the mirror will be given by the dual fibration. It is a folklore that the Ricci-flat metrics near large complex limits are approximated by semi-flat metrics with instanton correction related to the holomorphic discs with boundaries on special Lagrangian fibres [9]. The first part is done for K3 surfaces: [20] wrote down the semi-flat metric for the special Lagrangian fibration. Later, [19] proved that for elliptic K3 surfaces around large complex limits, the Ricci-flat metrics are approximated by the semi-flat metrics gluing with Ooguri-Vafa metrics. However, the instanton corrections are not included in [19]. Although the original problem is formulated as a differential geometric problem, [23],[18] had big success toward an algebraic-geometric version of SYZ conjecture. They incorporated the instanton problems of complex structure with the tropical geometry.

Inspiring by closed topological string theory, Gromov-Witten theory is a useful tool in probing algebraic geometry/ symplectic geometry and produces interesting enumerative invariants, counting number of curves with certain incidence conditions in the target space. One can also consider the open topological string analogue and try to define open Gromov-Witten invariants, counting (pseudo-)holomorphic Riemann surface with Lagrangian 
boundary conditions. However, it is hard to define open Gromov-Witten type invariants due to the existence of codimension one boundaries of the moduli spaces. For compact Calabi-Yau case, there are only two situations of which open Gromov-Witten invariants can be defined in the literature. One is the case when the Calabi-Yau admits an anti-symplectic involution and the Lagrangian is given by the fixed locus [33]. The other one is the case of Calabi-Yau 3-folds with rational homology sphere Lagrangian boundary condition [8]. Notice that in both situations the Lagrangian boundary conditions are rigid in certain sense. Here we will present a new type of open Gromov-Witten invariants on general hyperKähler manifolds and on CalabiYau 3-folds with K3 sibration in [30]. Moreover, if one wants to define open Gromov-Witten invariants on K3 surfaces, there is another naive obstruction by easy dimension count. Namely, the moduli space of holomorphic discs (with special Lagrangian boundary condition) has virtual dimension minus one. In particular, there is no pseudo-holomorphic discs with respect to a generic almost complex structure. In other words, even if we can get rid of the first issue and make the counting well-defined, the invariant would just be zero.

To solve the two difficulties mentioned above, we proposed to consider the Lagrangian boundary condition with non-trivial deformations. Given a hyperKähler manifold $X$ and a holomorphic Lagrangian submanifold $L$ with nontrivial deformation, any choice of hyperKähler metric will induced an $S^{1}$ family of complex structure making $L$ a special Lagrangian submanifold. It is natural to consider the moduli space of discs holomorphic with respect to these $S^{1}$-family of complex structures. The first observation is that given 
any relative homology class, there is at most one complex structure which makes it holomorphic. In other words, the new moduli space coincide with the usual one as topological spaces. However, after the introducing of $S^{1}$ family of complex structure on the target space, the virtual dimension of the new moduli space will be one dimension higher. Similar to the idea of changing tangent-obstruction theory to define reduced Gromov-Witten invariants in algebraic geometry, one can construct a new Kuranishi structure on the new moduli space using the $S^{1}$-family of complex structures. Via this new Kuranishi structure, one can construct a reduced $A_{\infty}$ structure on $\Lambda^{*}\left(L \times S_{\vartheta}^{1}\right)$ and a new virtual fundamental class if the moduli space has no codimension one boundary. We couple the symplectic area and the phase of the holomorphic discs to get a holomorphic function called central charge. The holomorphicity and Gromov compactness theorem will guarantee that the codimension one boundaries of the moduli spaces only occur as an real analytic Zariski closed subsets in the deformation space of $L$. Although the invariants cannot be defined when the bubbling phenomenon occur, we can interpret the locus where bubbling occurs as the walls of marginal stability in physics. We expect the invariants jump when the Lagrangian boundary conditions vary across the wall of marginal stability and the jump is governed by Kontsevich-Soibelman wall-crossing formula. Therefore the structure is similar to Donalson-Thomas theory in algebraic geometry, changing the boundary condition can be viewed as changing stability condition. The deformation space of the holomorphic Lagrangian $L$ can be viewed as a complex isotropic space of the stability manifold.

We first review the twistor construction of hyperKähler metric in [17] 
in section 2. Then we define the tropical counting invariants satisfying Kontsevich-Soibelman wall-crossing formula in section 3 and 4, thus realize the physics model of [17] in $\mathrm{K} 3$ case. In particular, we prove mirror symmetry in by identifying the tropical data and the instanton correction of complex structure of the mirror in the sense of [17]. Then we define an open Gromov-Witten type invariants on holomorphic Lagrangian torus of hyperKähler manifolds using de Rham model introduced in [7] in section 5 and establish the correspondence between tropical geometry and holomorphic geometry. We will present an non-trivial example of wall-crossing phenomenon at the end of section 5. We use this tropical discs counting to construct a quantum corrected SYZ transform on Ooguri-Vafa space in section 6 . In section 7 , we introduce a real version of Noether-Lefchetz theory and establish the relation between reduced disc counting on K3 and disc counting on Calabi-Yau 3-folds with K3 fibration.

\section{Review of Twistorial Construction}

\subsection{Settings and HyperKähler Rotation}

Let $X$ be a hyperKähler manifold of dimension $n$, then it admits a $\mathbb{P}^{1}$-family of complex structures, called twistor line parametrized by $\zeta$, given by

$$
J_{\zeta}=\frac{i(-\zeta+\bar{\zeta}) J_{1}-(\zeta+\bar{\zeta}) J_{2}+\left(1-|\zeta|^{2}\right) J_{3}}{1+|\zeta|^{2}}
$$

where $J_{1}, J_{2}$ and $J_{3}$ are integrable complex structures satisfying the quaternionic relation. Moreover, the holomorphic symplectic 2 -forms $\Omega_{\zeta}$ with re- 
spect to the compatible complex structure $J_{\zeta}$ are given by

$$
\Omega_{\zeta}=-\frac{i}{2 \zeta} \omega_{+}+\omega_{3}-\frac{i}{2} \zeta \omega_{-}
$$

where $\omega_{ \pm}=\omega_{1} \pm \omega_{2}$.

Remark 2.1. Let $L$ be a holomorphic Lagrangian in $(X, \omega, \Omega)$, namely, $\left.\Omega\right|_{L}=0$. Assume the north and south pole the twistor line is given by $(\omega, \Omega)$ and $(-\omega, \bar{\Omega})$ respectively, making $L$ an holomorphic Lagrangian. The hyperKähler structures corresponding to the equator $\{\vartheta=\zeta:|\zeta|=1\}$ provides a special Lagrangian $L$ in $X_{\vartheta}=\left(X, \omega_{\vartheta}, \Omega_{\vartheta}\right)$. In particular, if $(X, \omega, \Omega)$ admits holomorphic Lagrangian fibration, then it induces an $S^{1}$-family of special Lagrangian fibration on $X_{\vartheta}$ for each $\vartheta \in S^{1}$. This is the so-called hyperKähler rotation trick.

The twistor construction is based on the following characteristic properties of the twistor space of a hyperKähler manifold:

Theorem 2.2. [22] $X$ is a manifold and $\mathcal{Z}=X \times \mathbb{C P}^{1}$ admits a complex structure such $p: \mathcal{Z} \rightarrow \mathbb{C P}^{1}$ is holomophic and

1. There is a holomorphic section $\varpi$ of $\Omega_{\mathcal{Z} / \mathbb{C P}^{1}}^{2} \otimes \mathcal{O}(2)$ restricting to the holomorphic symplectic form $\Omega_{\zeta}$ on each fibre $p^{-1}(\zeta)$.

2. The map $\sigma(x, \zeta)=(x,-1 / \bar{\zeta})$ gives an anti-holomophic involution of $\mathcal{Z}$ , which coves the antipodal map on $\mathbb{C P}^{1}$, and preserves $\varpi$ in the sense that $\sigma^{*} \varpi=\bar{\varpi}$.

Then $X$ is the set of holomorphic curves $C$ in $\mathcal{Z}$ isomorphic to $\mathbb{C P}^{1}$ with normal bundles and preserved by the involution is a hyperKähler manifold. 
The above characteristic properties of the twister space of a hyperKähler manifold allow us to cook up a hyperKähler metric $g$ on a K3 surface $X$ from $\mathcal{Z}$. Since $\varpi^{n+1}=0$, it follows that $\omega_{+}^{n} \wedge \omega_{3}=0$. Therefore the real 2 -form $\omega_{3}$ is of type $(1,1)$ in complex structure $J_{3}$ and we can use $J_{3}$ and $\omega_{3}$ to build a Kähler metric $g$ on $X$. This $g$ is the hyperKähler metric guaranteed by the twister construction. Therefore, to extract the hyperKähler metric, it suffices to write down the holomorphic $(2,0)$-form $\Omega_{\zeta}$, for all $\zeta \in \mathbb{P}^{1}$.

Using this idea, [17] proposes a recipe of constructing holomorphic $(2,0)$ forms on the semi-flat part of a hyperKähler manifold with holomorphic Lagrangian fibration. We need the following data as input:

1. A complex manifold $B$ with a divisor $D$ and $B_{0}=B \backslash D$, where $B$ is the base of the holomorphic Lagrangian fibration and $D$ will be the discriminant locus of the torus fibration later. $B$ is also referred as the Coulumb branch of abelian $\mathcal{N}=2$ gauge theory.

2. A local system $\Gamma_{g}$ over $B_{0}$, with a rank 2 lattice equipped with a non-degenerate anti-symmetric integer-valued pairing $\langle$,$\rangle .$

3. A fixed lattice $\Gamma_{f}$ (possibly trivial).

4. A local system $\Gamma$ of lattices over $B_{0}$, given by the extension

$$
0 \rightarrow \Gamma_{f} \rightarrow \Gamma \rightarrow \Gamma_{g} \rightarrow 0
$$

The pairing $\langle$,$\rangle on \Gamma_{g}$ induces one on $\Gamma$ (also denoted by $\langle$,$\rangle ) with$ radical $\Gamma_{f}$. 
5. A homomorphism $Z: \Gamma \rightarrow \mathbb{C}$, varying holomorphic over $B_{0}$. For any local section $\gamma$ of $\Gamma$ we have a local holomorphic function over $B_{0}$. We call $Z$ the central charge.

6. An element $\theta_{f} \in \Gamma_{f}^{*} \otimes(\mathbb{R} / 2 \pi \mathbb{Z})$.

The above data should satisfy the conditions below:

1. $Z_{\gamma_{f}}$ is a constant function for every $\gamma_{f} \in \Gamma_{f}$.

2. $\langle d Z, d Z\rangle=0$ and $\langle d Z, d \bar{Z}\rangle>0$.

3. For any $u \in B_{0}$, the $d Z_{\gamma}(u)$ span $T_{u}^{*} B_{0}$.

Remark 2.3. The above data determine an $S^{1}$-family of affine structures on $B_{0}$. For any $\vartheta \in \mathbb{R} / 2 \pi \mathbb{Z}$, the functions $f_{i}=\operatorname{Re}\left(e^{i \vartheta} Z_{\gamma_{i} \in \Gamma_{g}}\right)$, give the local integral affine coordinates with transition functions in $S p(2, \mathbb{Z}) \ltimes \mathbb{R}^{2}$. In particular, Neither the choice of Kähler class of the elliptic K3 nor the scaling of the holomorphic $(2,0)$-form change the affine straight lines on the base affine manifold.

From the above data, we can construct the semi-flat part of an hyperKähler manifold with holomorphic Lagrangian fibration. Indeed, let $\operatorname{TChar}_{u}\left(\Gamma, \theta_{f}\right)$ be the set of twisted unitary characters of $\Gamma_{u}$, namely, $\theta$ : $\Gamma_{u} \rightarrow \mathbb{R} / 2 \pi \mathbb{Z}$ satisfying

$$
\theta_{\gamma}+\theta_{\gamma^{\prime}}=\theta_{\gamma+\gamma^{\prime}}+\pi\left\langle\gamma, \gamma^{\prime}\right\rangle
$$

and agree with $\theta_{f}$ when restricting on $\Gamma_{f} \subseteq \Gamma$. Each fibre $\operatorname{TChar}_{u}\left(\Gamma, \theta_{f}\right) \cong$ $\left(S^{1}\right)^{n}$ and they glue together to recover the torus bundle $X_{0}$ over $B_{0}$. 


\subsection{BPS Counting and Wall-Crossing Formula}

Let $\mathfrak{T}$ be the complexified symplectic torus with coordinate $x_{i}$ and equipped with a standard holomorphic symplectic 2-form

$$
\varpi=\sum \epsilon_{i j} \frac{d X_{i}}{X_{j}} \wedge \frac{d X_{j}}{X_{j}}
$$

where $X_{i}$ are standard multiplicative coordinate on $\mathfrak{T}$. Our goal is to construct a multiplicative map $\chi: L_{u} \rightarrow \mathfrak{T}$. The pull-back of the standard symplectic 2-form $\chi^{*} \varpi$ gives a closed 2-form on $L_{u}$. Varying the base parameter $u \in B_{0}$ gives a global 2-form which is closed and non-degenerate when $R$ is large. There is an canonical smooth choice given by

$$
\chi_{\gamma}^{s f}(\zeta)=\exp \left[\pi R \frac{Z_{\gamma}}{\zeta}+i \theta_{\gamma}+\pi R \zeta \bar{Z}_{\gamma}\right]
$$

However, the resulting 2-form will induce the semi-flat metric by Theorem 2.2 and cannot be extended to the singular fibres because of the blow-up curvature. To overcome this defect, we need to add "instanton corrections" to $\chi_{\gamma}^{s f}$ : we introduce the generalized Daonaldson-Thomas invariant $\Omega: \Gamma \rightarrow$ $\mathbb{Z}$ satisfying $\Omega(\gamma ; u)=\Omega(-\gamma ; u)$. Moreover, to each $\gamma \in \Gamma_{u}$ one associates a birational Poisson automorphism $\mathcal{K}_{\gamma}$ of $\mathfrak{T}$, defined by

$$
\mathcal{K}_{\gamma}^{*} X_{\gamma^{\prime}}=X_{\gamma^{\prime}}\left(1-X_{\gamma}\right)^{\left\langle\gamma, \gamma^{\prime}\right\rangle}
$$

We attach a BPS ray to each $\gamma \in \Gamma_{u}$,

$$
l_{\gamma}(u):=Z_{\gamma}(u) \mathbb{R}_{-} .
$$


Then for each ray in the form above in the $\zeta$-plane, we associate a birational Poisson automorphism of $\mathfrak{T}$,

$$
S_{l}(u):=\prod_{\gamma: l_{\gamma}(u)=l} \mathcal{K}_{\gamma}^{\Omega(\gamma ; u)}
$$

Define an anti-holomorphic involution $\tau$ of $\mathfrak{T}$ by

$$
\tau^{*} X_{\gamma}=\bar{X}_{-\gamma}
$$

The Riemann-Hilbert problem is formulated as follows : Fix $u \in B_{0}$, our goal is to find a map $\chi: X \times \mathbb{C}^{*} \rightarrow \mathfrak{T}$. with the properties below:

1. $\chi$ is piecewise-holomorphic on $\zeta \in \mathbb{C}^{*}$, with discontinuities only along the BPS rays $l_{\gamma}(u)$ for each $\gamma \in \Gamma_{u}$ and $\Omega(\gamma ; u) \neq 0$.

2. The limits $\chi^{ \pm}$of $\chi$ as $\zeta$ approaches any BPS ray $l$ from both sides exist and are related by

$$
\chi^{+}=S_{l}^{-1} \circ \chi^{-}
$$

3. Reality condition

$$
\chi(-1 / \bar{\zeta})=\tau^{*} \chi(\zeta)
$$

4. For any $\gamma$, the limit $\lim _{\zeta \rightarrow 0} \chi_{\gamma}(\zeta) / \chi_{\gamma}^{s f}(\zeta)$ exists and is real.

We will focus on the first two properties above in this paper.

Definition 2.4. The wall of marginal stability $W$ is a real codimension one 
subset on $B_{0}$ given by

$$
W=\bigcup W_{\gamma_{1}, \gamma_{2}}
$$

where

$$
W_{\gamma_{1}, \gamma_{2}}=\left\{u: \exists \gamma_{1}, \gamma_{2} \text { with } \Omega\left(\gamma_{1} ; u\right) \neq 0, \Omega\left(\gamma_{2} ; u\right) \neq 0, \frac{Z_{\gamma_{1}}(u)}{Z_{\gamma_{2}}(u)} \in \mathbb{R}_{+}\right\}
$$

Choose a strictly convex cone $V$ in $\mathbb{C}$ with apex at the origin, then for each $u \notin W$ we define

$$
A_{V}(u)=\prod_{\gamma: Z_{\gamma}(u) \in V} \mathcal{K}_{\gamma}^{\Omega(\gamma ; u)}=\prod_{l \in V} S_{l}(u)
$$

where the product is taken in order of increasing $\operatorname{Arg} Z_{\gamma}(u)$.

We say the set of numbers $\{\Omega(\gamma ; u)\}$ satisfy Kontsevich-Soibelman wallcrossing formula if given a path in $B_{0}$ connecting $u$ and $u^{\prime}$ which has no point $u$ with $Z_{\gamma}(u) \in \partial V$ and $\Omega(\gamma ; u) \neq 0$, then $A_{V}(u)$ and $A_{V}\left(u^{\prime}\right)$ are related by parallel transport in $B_{0}$ along the path.

Remark 2.5. The smoothness of 2-form $\chi^{*} \varpi$ is interpreted as KontsevichSoibelman wall-crossing formula in [17].

From the wall-crossing formula, $\Omega(\gamma, u)$ are locally constant on $B_{0} \backslash W$. Any path passing through a generic point $u$ of a wall $W_{\gamma_{1}, \gamma_{2}}$ with $d \frac{Z_{\gamma_{1}}}{Z_{\gamma_{2}}}(u) \neq$ 0 , then the phase of $Z_{\gamma_{1}}$ and $Z_{\gamma_{2}}$ change order when we change $u$ across the wall of marginal stability. To make the product $A_{V}(u)$ unchanged, $\Omega(\gamma ; u)$ may jump when crossing the wall $W$. One would be able to solve for all 
$\Omega(\gamma ; u)$, if we know $\Omega\left(\gamma ; u_{0}\right)$ for some fixed $u_{0}$ (see Theorem 5.50).

\subsection{Solving the Riemann-Hilbert Problem}

From the Corollary 2.5, we want to solve the following functional equation to glue the local holomorphic 2 -forms to a global one for $\zeta \in \mathbb{C}^{*}$.

$$
\chi_{\gamma}(\zeta)=\chi_{\gamma}^{s f}(\zeta) \exp \left[\frac{1}{4 \pi i} \sum_{l} \int_{l} \frac{d \zeta^{\prime}}{\zeta^{\prime}} \frac{\zeta^{\prime}+\zeta}{\zeta^{\prime}-\zeta} \log \frac{\chi_{\gamma}\left(e^{-i \epsilon} \zeta^{\prime}\right)}{\left(\chi S_{l}\right)_{\gamma}\left(e^{-i \epsilon} \zeta^{\prime}\right)}\right]
$$

From the explicit form of the Kontsevich-Soibelman factor, we have

$$
\left(\chi S_{l}\right)_{\gamma}=\chi_{\gamma} \prod_{\gamma^{\prime}}\left(1-\sigma\left(\gamma^{\prime}\right) \chi_{\gamma^{\prime}}\right)^{\Omega\left(\gamma^{\prime} ; u\right)\left\langle\gamma, \gamma^{\prime}\right\rangle}
$$

Therefore, the integral formula for $\chi$ becomes

$$
\chi_{\gamma}(\zeta)=\chi_{\gamma}^{s f}(\zeta) \exp \left[-\frac{1}{4 \pi i} \sum_{\gamma^{\prime}} \Omega\left(\gamma^{\prime} ; u\right)\left\langle\gamma, \gamma^{\prime}\right\rangle \int_{l} \frac{d \zeta^{\prime}}{\zeta^{\prime}} \frac{\zeta^{\prime}+\zeta}{\zeta^{\prime}-\zeta} \log \left(1-\sigma\left(\gamma^{\prime}\right) \chi_{\gamma^{\prime}}\right)\right]
$$

We will solve the above integral equation by iteration with initial data $\chi=\chi^{s f}$. We first introduce $\mathbb{Q}$-valued invariants related to $\Omega(\gamma)$ by the "multiple cover formula" (see also [28])

$$
\tilde{\Omega}(\gamma)=\sum_{n=1}^{\infty} \frac{\Omega(\gamma / n)}{n^{2}} .
$$

Here we define $\Omega(\gamma / n)=0$ if $n$ does not divide $\gamma$. Equivalent,

$$
\Omega(\gamma)=\sum_{n=1}^{\infty} \mu(n) \frac{\tilde{\Omega}(\gamma / n)}{n^{2}}
$$


by Mobius inversion formula.

Then we consider rooted trees with vertices labeled by charges $\gamma_{i} \in \Gamma$ and edges labeled by pairs $(i, j)$ (where $i$ is the node closer to the root). For each such tree $\mathcal{T}$, we associate a weight

$$
\tilde{\Omega}(\mathcal{T})=\frac{1}{|A u t(\mathcal{T})|} \prod_{i \in v(\mathcal{T})} \tilde{\Omega}\left(\gamma_{i}\right) \prod_{(i, j) \in E(\mathcal{T})}\left\langle\gamma_{i}, \gamma_{j}\right\rangle
$$

Let $\gamma_{\mathcal{T}}$ denote the label at the root vertex of $\mathcal{T}$. We define a function $\mathcal{G}_{\mathcal{T}}(\zeta)$ on ( a patch of ) $M$ inductively as follows: deleting the root vertex from $\mathcal{T}$ leaves behind a set of trees $\mathcal{T}_{a}$, and set

$$
\mathcal{G}_{\mathcal{T}}(\zeta)=\frac{1}{4 \pi i} \int_{l_{\gamma_{\mathcal{T}}}} \frac{d \zeta^{\prime}}{\zeta^{\prime}} \frac{\zeta^{\prime}+\zeta}{\zeta^{\prime}-\zeta} \chi_{\gamma_{\mathcal{T}}}^{s f}\left(\zeta^{\prime}\right) \prod_{a} \mathcal{G}_{\mathcal{T}_{a}}\left(\zeta^{\prime}\right)
$$

Proposition 2.6. The formal solution for the iteration integral equation (3) is

$$
\chi_{\gamma}(\zeta)=\chi_{\gamma}^{s f}(\zeta) \exp \left[\sum_{\mathcal{T}}\left\langle\gamma, \gamma_{\mathcal{T}}\right\rangle \tilde{\Omega}(\mathcal{T}) \mathcal{G}_{\mathcal{T}}(\zeta)\right]
$$

Proof. Let $\chi_{\gamma}^{(0)}=\chi_{\gamma}^{s f}$ and define iteration by

$$
\chi_{\gamma}^{(i+1)}(\zeta)=\chi_{\gamma}^{s f}(\zeta) \exp \left[\frac{-1}{4 \pi i} \sum_{\gamma^{\prime}} \Omega\left(\gamma^{\prime} ; u\right)\left\langle\gamma, \gamma^{\prime}\right\rangle \int_{l_{\gamma^{\prime}(u)}} \frac{d \zeta^{\prime}}{\zeta^{\prime}} \frac{\zeta+\zeta^{\prime}}{\zeta-\zeta^{\prime}} \log \left(1-\chi_{\gamma^{\prime}}^{(i)}\left(\zeta^{\prime}\right)\right)\right]
$$


Formally, we can compute $\chi_{\gamma}^{(i)}$ by induction

$$
\begin{aligned}
& \chi_{\gamma}^{(i+1)}(\zeta)=\chi_{\gamma}^{s f}(\zeta) \exp \left[\frac{1}{4 \pi i} \sum_{\gamma^{\prime}} \Omega\left(\gamma^{\prime} ; u\right)\left\langle\gamma, \gamma^{\prime}\right\rangle \int_{l_{\gamma^{\prime}(u)}} \frac{d \zeta^{\prime}}{\zeta^{\prime}} \frac{\zeta+\zeta^{\prime}}{\zeta-\zeta^{\prime}} \sum_{k=1}^{\infty} \frac{\chi_{k \gamma^{\prime}}^{(i)}\left(\zeta^{\prime}\right)}{k}\right] \\
& =\chi_{\gamma}^{s f}(\zeta) \exp \left[\frac{1}{4 \pi i} \sum_{\gamma^{\prime}} \sum_{k=1}^{\infty} \frac{\Omega\left(\gamma^{\prime} ; u\right)}{k^{2}}\left\langle\gamma, k \gamma^{\prime}\right\rangle \int_{l_{\gamma^{\prime}(u)}} \frac{d \zeta^{\prime}}{\zeta^{\prime}} \frac{\zeta+\zeta^{\prime}}{\zeta-\zeta^{\prime}}\right. \\
& \left.\chi_{k \gamma^{\prime}}^{s f}\left(\zeta^{\prime}\right) \exp \left[\sum_{\mathcal{T}} \tilde{\Omega}\left(\gamma^{\prime \prime}\right)\left\langle k \gamma^{\prime}, \gamma^{\prime \prime}\right\rangle \mathcal{G}_{\gamma^{\prime \prime}}\left(\zeta^{\prime}\right)\right]\right] \\
& =\chi_{\gamma}^{s f}(\zeta) \exp \left[\frac{1}{4 \pi i} \sum_{\gamma^{\prime}} \tilde{\Omega}\left(\gamma^{\prime}\right)\left\langle\gamma, \gamma^{\prime}\right\rangle \int_{l_{\gamma^{\prime}(u)}} \frac{d \zeta^{\prime}}{\zeta^{\prime}} \frac{\zeta+\zeta^{\prime}}{\zeta-\zeta^{\prime}}\right. \\
& \left.\chi_{\gamma^{\prime}}^{s f}\left(\zeta^{\prime}\right) \exp \left[\sum_{\mathcal{T}: \operatorname{dept}(\mathcal{T}) \leq i} \tilde{\Omega}(\mathcal{T})\left\langle\gamma^{\prime}, \gamma_{\mathcal{T}}\right\rangle \mathcal{G}_{\mathcal{T}}\left(\zeta^{\prime}\right)\right]\right] \\
& =\chi_{\gamma}^{s f}(\zeta) \exp \left[\frac{1}{4 \pi i} \sum_{\gamma^{\prime}} \tilde{\Omega}\left(\gamma^{\prime}\right)\left\langle\gamma, \gamma^{\prime}\right\rangle \int_{l_{\gamma^{\prime}(u)}} \frac{d \zeta^{\prime}}{\zeta^{\prime}} \frac{\zeta+\zeta^{\prime}}{\zeta-\zeta^{\prime}}\right. \\
& \left.\chi_{\gamma^{\prime}}^{s f}\left(\zeta^{\prime}\right) \sum_{s=0}^{\infty} \frac{\left(\sum_{\mathcal{T}: \operatorname{dept}(\mathcal{T}) \leq i} \tilde{\Omega}(\mathcal{T})\left\langle\gamma^{\prime}, \gamma_{\mathcal{T}}\right\rangle \mathcal{G}_{\mathcal{T}}\left(\zeta^{\prime}\right)\right)^{s}}{s !}\right] \\
& =\chi_{k \gamma^{\prime}}^{s f}\left(\zeta^{\prime}\right) \exp \left[\sum_{\mathcal{T}: \operatorname{dept}(\mathcal{T}) \leq i+1}\left\langle\gamma, \gamma_{\mathcal{T}}\right\rangle \tilde{\Omega}(\mathcal{T}) \mathcal{G}_{\mathcal{T}}(\zeta)\right]
\end{aligned}
$$

In the first equality we use the Taylor expansion of $\log (1-x)$ and the fact that $\left(\chi_{\gamma}^{(i)}\right)^{k}=\chi_{k \gamma}^{(i)}$, which is valid because $\chi_{\gamma}^{s f}$ is strictly negative along the BPS rays $l_{\gamma(u)}$. The second equality we use the induction hypothesis and the third "equality" we use a formal resummation formula

$$
\sum_{\gamma^{\prime}} \sum_{k=1}^{\infty} \frac{\Omega\left(\gamma^{\prime} ; u\right)}{k^{2}} f\left(k \gamma^{\prime}\right)=\sum_{\gamma^{\prime}} f\left(\gamma^{\prime}\right) \tilde{\Omega}\left(\gamma^{\prime}\right)
$$

The fourth "equality" we use the Taylor expansion for $e^{x}$ and another resummation (7) in the last "equality". 
Remark 2.7. [17] If $|\Omega(\gamma)| \leq e^{\alpha\|\gamma\|}$ for some constant $\alpha$, then the above formal sum equalities converge absolutely and the twistor construction provides $a \mathbb{C}^{*}$-family of holomorphic 2-forms OUTSIDE the singular fibres.

Remark 2.8. The formal expansion expression (6) can help to establish SYZ transform on Ooguri-Vafa space (see section 6).

Remark 2.9. There is a symmetry $\zeta \mapsto e^{i \vartheta} \zeta$, and $Z \mapsto e^{i \vartheta} Z$ on (6). Also, we have $\chi_{-\gamma}(-1 / \bar{\zeta})=\overline{\chi_{\gamma}(\zeta)}$ formally.

\subsection{Local example: Ooguri-Vafa metric}

We will follow the setting for the recipe above:

1. Choose $B=\{|u|<\Lambda\}$ be a disc and the discriminant locus is just the origin.

2. $\Gamma=\Gamma_{g}$ is a rank-2 local system of lattices over $B_{0}$, with monodromy around the origin $\gamma_{e} \rightarrow \gamma_{e}, \gamma_{m} \rightarrow \gamma_{e}+\gamma_{m}$ after choosing a special set of local basis of sections $\left(\gamma_{e}, \gamma_{m}\right)$.

3. The intersection pairing $\left\langle\gamma_{e}, \gamma_{m}\right\rangle=1$.

4. The central charges are $Z_{\gamma_{e}}=u$ and $Z_{\gamma_{m}}=u \log \frac{u}{\Lambda}-u$. (Note that both are globally defined.)

5. $\Gamma_{f}$ and $\theta_{f}$ are trivial.

6. For all $u$, we have $\Omega(\gamma, u)= \begin{cases}1 & \text { if } \gamma= \pm \gamma_{e} \\ 0 & \text { otherwise }\end{cases}$ 
Following the recipe, one can derive Ooguri-Vafa metric [31] from the above input data.

Remark 2.10. This is so far the only example of the holomorphic 2-forms constructed from Gaiotto-Moore-Neitzke's recipe which can extend over singular fibres (not even the pentagon example). In Ooguri-Vafa case, the extension follows from Poisson summation formula.

One can construct an $S^{1}$-family of special Lagrangian fibration on the total space of above elliptic fibration $X$ for each $\zeta$, with $|\zeta|=1$ by

$$
T_{s, \lambda}=\left\{\left(u_{1}, u_{2}, \theta_{e}, \theta_{m}\right) \in X_{\zeta}: \log \left|\chi_{e}(\zeta)\right|=s, \mu_{S^{1}}=\lambda\right\}
$$

Remark 2.11. [4] By maximum principle, $T_{s, \lambda}$ bounds a holomorphic disc if and only if $\operatorname{Re}(u \bar{\zeta})=0, u=u_{1}+i u_{2}$.

The two generators of $\Gamma=H_{2}(X, T) \cong H_{2}(T)$ can be written down explicitly. $\gamma_{1}$ is the initial disc, which is formed by the collection of $\left\{\theta_{e}=\right.$ const. $\}$ in the fibres of $\chi_{e}(\zeta): X \rightarrow \mathbb{C}$ along the segment to origin. $\gamma_{2}$ is a section of $\chi_{e}(\zeta)$ restricted to $\left\{\lambda=-\frac{2 \pi}{\epsilon} b_{1}=\right.$ const. $\}$.

Since the mirror of $X_{-1}$ is $X_{i}$, direct calculation shows the following:

Observation 2.12. The central charge $Z: \Gamma \rightarrow \mathbb{C}$ is given by the integration of the 2 -form $\omega+i \operatorname{Im} \Omega$ on its mirror.

Remark 2.13. In [31], the total space of Ooguri-Vafa space is interpreted as part of the hypermultiplet moduli space of type II string compactification on a Calabi-Yau threefold. The coordinate $y=\int \Omega$ is the period of vanishing 
cycle, where the classical hypermultiplet moduli space develops a singularity at $y \rightarrow 0$. The other coordinate $t$ and $u$ are the expectation values of the $R R$ 3-form corresponding to the vanishing cycle and its dual.

\subsection{Application to Elliptic K3 Surfaces}

We would start with $f: X \rightarrow B$ an elliptic K3 surface (with a holormorphic section) and a prescribed Kähler class $[\omega]$. From Yau's theorem there exists a unique Ricci-flat Kähler form $\omega$ satisfying the Monge-Ampere equation $2 \omega^{2}=\Omega \wedge \bar{\Omega}$, where $\Omega$ is a non-vanishing holomorphic $(2,0)$-form. The triple $(X, \omega, \Omega)$ will induce a twistor family of K3 surfaces. For the input of above twistor construction, we will use the long exact sequence

$$
H_{2}(X) \rightarrow \Gamma=H_{2}\left(X, L_{u}\right) \rightarrow \Gamma_{g}=H_{1}\left(L_{u}\right) \rightarrow 0,
$$

The symplectic pairing $\langle$,$\rangle is taken to be the natural pairing of homology on$ $H_{1}\left(J_{u}\right)$. The central charge $Z$ is taken to be the period $\gamma \mapsto \int_{\gamma} \Omega$, for each $\gamma \in H_{2}\left(X, L_{u}\right)$. The integral is well-defined because $\left.\Omega\right|_{L}=0$. The following lemma is straight forward computation:

Lemma 2.14. For any $v \in T B_{0}$, we have

$$
d Z_{\gamma}(v)=\int_{\partial \gamma} \iota_{\tilde{v}} \Omega
$$

where $\tilde{v} \in T X$ is any lifting of $v$.

Proof. Since $\left.\Omega\right|_{L}=0$, we view $\Omega$ as the element $(\Omega, 0) \in H^{2}(X, L)$. From 
the variational formula of relative pairing,

$$
\begin{aligned}
d Z_{\gamma}(v) & =\mathcal{L}_{v}\langle\gamma,(\Omega, 0)\rangle \\
& =\left\langle\gamma,\left(\iota_{\tilde{v}} d \Omega, \iota_{\tilde{v}}(0-\Omega)\right)\right\rangle=\int_{\partial \gamma} \iota_{\tilde{v}} \Omega
\end{aligned}
$$

Given a point $u_{0} \in B_{0}$ and an element $\gamma_{u_{0}} \in \Gamma_{u_{0}}$, there exists a neighborhood $\mathcal{U}$ of $u_{0}$ and a neighborhood of $\tilde{\mathcal{U}}$ of $\gamma_{u_{0}}$ such that $\mathcal{U}$ is homeomorphic to $\tilde{\mathcal{U}}$. Under this identification, we have

Corollary 2.15. The central charge $Z: \Gamma \rightarrow \mathbb{C}$ is a holomorphic function on $\Gamma$.

Proof. Since any $(0,1)$-vector on $T B_{0}$ can be expressed in term of $v+i J v$ for some $v \in T B_{0}$,

$$
(v+i J v) Z=\int_{\partial \gamma} \iota_{(\tilde{v}+i J \tilde{v})} \Omega=0
$$

The latter equality holds because $\Omega$ is a $(2,0)$-form and $\tilde{v}+i J \tilde{v}$ is a $(0,1)$ vector. Notice that for $y$ near a singularity of the affine structure, $\gamma$ represents the relative class of Lefschetz thimble, then $Z_{\gamma}$ is bounded in a neighborhood of the singularity and thus is a removable singularity.

Because both $\operatorname{Re} \Omega$ and $\operatorname{Im} \Omega$ are symplectic form, another immediate consequence of Lemma 2.14 is the following:

Corollary 2.16. Let $\gamma \in \Gamma$, then $d Z_{\gamma} \neq 0$ whenever $Z_{\gamma}$ is defined. 
Corollary 2.17. $\langle d Z, d Z\rangle=0$ and $\langle d Z, d \bar{Z}\rangle>0$.

Proof. There is a standard short exact sequence

$$
0 \rightarrow R^{1} f_{*} \mathbb{Z} \rightarrow R^{1} f_{*} \mathcal{O}_{X} \cong \omega_{\mathbb{P}^{1}} \rightarrow \mathcal{O}^{\#} \rightarrow 0
$$

where $\mathcal{O}^{\#}$ denotes the sheaf of holomorphic sections of $f: X \rightarrow \mathbb{P}^{1}$. Here $R^{1} f_{*} \mathcal{O}_{X}$ is identified as the normal bundle of the zero section and the last map is the fibrewise exponential map. There is a natural holomorphic symplectic 2-form $\Omega_{\text {can }}$ on $R^{1} f_{*} \mathcal{O}_{X}$ and descend to the quotient. On the other hand, any holormophic symplectic 2 -form is a multiple of $\Omega_{\text {can }}$. Therefore, the holomorphic volume form of an elliptic K3 surface coincides with the one descending from the canonical volume form of the cotangent bundle of the base. The proposition follows from direct computations and Lemma 2.14 .

Remark 2.18. In particular, the non-vanishing holomorphic 2-form of an elliptic K3 surface receive no quantum correction and admits local $S^{1}$-action near singularities and local $T^{2}$-action away from singularities.

In particular, we have $Z_{\gamma}(u) \neq 0$ for $\Omega(\gamma) \neq 0$ unless $u$ is the singular point of the affine structure and $\gamma$ is multiple of the Lefschetz thimble.

To apply the twistor construction to elliptic K3 surfaces, we still need the key ingredient: the generalized Donaldson-Thomas invariants $\Omega(\gamma)$ which we will discuss in the next section. 


\section{Scattering Diagrams and Generalized Donaldson- Thomas Invariants on K3 Surfaces}

\subsection{Construction of Scattering Diagrams on Elliptic K3 Sur- faces}

We first introduce a version of Novikov ring. Let $R$ be a commutative ring with unit,

$$
\Lambda_{0}(R)=\left\{\sum_{i=0}^{\infty} a_{i} T^{\lambda_{i}} \mid a_{i} \in R, \lambda \in \mathbb{R}_{\geq 0}, \lim _{i \rightarrow \infty} \lambda_{i}=\infty\right\}
$$

and

$$
\Lambda_{+}(R)=\left\{\sum_{i=0}^{\infty} a_{i} T^{\lambda_{i}} \in \Lambda_{0}(R) \mid \lambda>0\right\}
$$

There is a natural filtration on $\Lambda_{0}(R)$ given by

$$
F^{\lambda} \Lambda_{0}(R)=T^{\lambda} \Lambda_{0}(R)
$$

for each $\lambda \in \mathbb{R}_{\geq 0}$. We will take $R=\mathbb{C}$ later on and ignore the notation $R$. The localization of $\Lambda_{0}$ at its maximal ideal, which is a generalization of puiseux series, is algebraically closed and complete in $T$-adic topology.

We define the module of $\log$ derivations of

$$
\mathbb{C}\left[\Gamma_{g}\right] \hat{\otimes}_{\mathbb{C}} \Lambda_{0}=\lim _{\leftarrow} \mathbb{C}\left[\Gamma_{g}\right] \otimes_{\mathbb{C}} \Lambda_{0} / F^{\lambda} \Lambda_{0}
$$


to be

$$
\Theta\left(\mathbb{C}\left[\Gamma_{g}\right] \hat{\otimes}_{\mathbb{C}} \Lambda_{0}\right)=\operatorname{Hom}\left(\Gamma_{g}, \mathbb{C}\left[\Gamma_{g}\right] \hat{\otimes}_{\mathbb{C}} \Lambda_{0}\right)=\left(\mathbb{C}\left[\Gamma_{g}\right] \hat{\otimes}_{\mathbb{C}} \Lambda_{0}\right) \otimes_{\mathbb{Z}} \Gamma_{g}^{*}
$$

For each element $a \partial_{n}=a \otimes n \in\left(\mathbb{C}\left[\Gamma_{g}\right] \hat{\otimes}_{\mathbb{C}} \Lambda_{0}\right) \otimes_{\mathbb{Z}} \Gamma_{g}^{*}$, it induces an ordinary derivation of $\mathbb{C}\left[\Gamma_{g}\right] \hat{\otimes}_{\mathbb{C}} \Lambda_{0}$ over $\Lambda_{0}$,

$$
\left(a \partial_{n}\right)\left(z^{\gamma}\right)=a\langle\gamma, n\rangle z^{\gamma}
$$

Let $\mathfrak{g}=\Lambda_{+}\left(\Theta\left(\mathbb{C}\left[\Gamma_{g}\right] \hat{\otimes}_{\mathbb{C}} \Lambda_{0}\right)\right)$. Given any $\xi \in \mathfrak{g}$, we have an element

$$
\exp (\xi) \in A u t_{\Lambda_{0}}\left(\mathbb{C}\left[\Gamma_{g}\right] \hat{\otimes}_{\mathbb{C}} \Lambda_{0}\right)
$$

From Baker-Cambell-Hausdorf formula and Lie algebra structure on $\mathfrak{g}$ given by

$$
\left[z^{\gamma} \partial_{n}, z^{\gamma^{\prime}} \partial_{n^{\prime}}\right]=(-1)^{\left\langle\gamma, \gamma^{\prime}\right\rangle} z^{\gamma+\gamma^{\prime}} \partial_{\left\langle\gamma^{\prime}, n\right\rangle n^{\prime}-\left\langle\gamma, n^{\prime}\right\rangle n}
$$

Remark 3.1. If we write $e_{\gamma}=z^{\gamma} \partial_{w_{\gamma} \gamma^{\perp}}, \gamma=\omega_{\gamma} \gamma_{\text {prim }}$ then the Lie bracket becomes

$$
\left[e_{\gamma_{1}}, e_{\gamma_{2}}\right]=(-1)^{\left\langle\gamma, \gamma^{\prime}\right\rangle}\left\langle\gamma, \gamma^{\prime}\right\rangle e_{\gamma+\gamma^{\prime}}
$$

The subset

$$
G=\{\exp \xi \mid \xi \in \mathfrak{g}\}
$$

is a subgroup of $A u t_{\Lambda_{0}}\left(\mathbb{C}\left[\Gamma_{g}\right] \hat{\otimes}_{\mathbb{C}} \Lambda_{0}\right)$. In particular, the subspace

$$
\mathfrak{h}=\bigoplus_{\gamma \in \Gamma \backslash\{0\}} z^{\gamma}\left(\Lambda_{+} \otimes \gamma^{\perp}\right)
$$


is closed under the above bracket and thus via exponential map produces a subgroup

$$
\mathbb{V}_{\text {trop }} \in G
$$

Lemma 3.2. The elements in $\mathbb{V}_{\text {trop }}$ induce sympectormorphisms of the complexified symplectic torus.

Proof. It suffices to prove that the generators $a$ of $\mathbb{V}_{\text {trop }}$ gives Hamilitionian vector field. Write $\Omega=d \log e_{1} \wedge d \log e_{2}$. and $\gamma=a_{1} e_{1}+a_{2} e_{2}$.

$$
\begin{aligned}
-z^{\gamma} \iota\left(z^{\gamma} \partial_{\gamma}\right) \Omega & =-z^{\gamma}\left(\left\langle z^{\gamma} \partial_{\gamma}, e_{1}\right\rangle d \log e_{2}-\left\langle z^{\gamma} \partial_{\gamma}, e_{2}\right\rangle d \log e_{1}\right) \\
& =-z^{\gamma}\left(-a_{2} d \log _{e_{2}}-a_{1} d \log e_{1}\right) \\
& =z^{\gamma} d \log r(m) \\
& =d\left(z^{\gamma}\right)
\end{aligned}
$$

Given an elliptic K3 surface $(X, \omega, \Omega)$ with Ricci-flat Kähler form $\omega$. After hyperKäher rotation, it induces an $S^{1}$-family of special Lagrangian torus fibration on $X_{\vartheta}$, for each $\vartheta \in S^{1}$ (see Remark 2.1). Fix a phase parameter $\vartheta \in S^{1}$ and we have an affine structure with singularities on the base $B$. We denote the discriminate locus by $\Delta$ and $B_{0}=B \backslash \Delta$.

Definition 3.3. A scattering diagram $\mathfrak{D}=\left\{\left(\mathfrak{d}_{i}, f_{i}\right)\right\}_{i \in I}$ on an integral affine manifold $B$ is a collection of 2-tuples such that

1. $\mathfrak{d}_{i}=o_{i}+\mathbb{R} m_{i}$ is a ray emanating from $o_{i} \in B_{0}$ with rational slope with respect to the affine structure, where $m_{i}$ is a primitive vector. 
2. The slab function $f_{i}(u) \in \mathbb{C}\left[z^{m_{i, u}}\right] \hat{\otimes}_{\mathbb{C}} \Lambda_{+}$for each $x \in \mathfrak{d}_{i}$, where $m_{i, u}$ is the parallel transport of $m_{i}$ from $o_{i}$ to $u$ along $\mathfrak{d}_{i}$. Moreover, each monomial of $f_{i}(u)$ is of the form $c z^{l m_{i, u}} T^{A(u)}$, where $c$ is a constant, $A(u)$ is a positive affine function along $\mathfrak{d}_{i}$ and $A\left(o_{i}\right)>0$.

3. For every point $u \in B$ and a given $\lambda>0$, there are only finitely many rays $\left(\mathfrak{d}_{i}, f_{i}\right) \in \mathfrak{D}$ such that $f_{i}(u) \not \equiv 0\left(\bmod T^{\lambda}\right)$.

4. The singularity of the scattering diagram $\operatorname{Sing}(\mathfrak{D})^{>\lambda}$ is given by the set

$$
\begin{array}{r}
\left\{u \in B \mid \exists\left(\mathfrak{d}_{i}, f_{i}\right) \in \mathfrak{D}, i=1,2 \text { such that } u \in \mathfrak{d}_{1} \cap \mathfrak{d}_{2}\right. \\
\text { and } \left.f_{1}(u) f_{2}(u) \not \equiv 0\left(\bmod T^{\lambda}\right)\right\}
\end{array}
$$

Let $\mathfrak{D}$ is a scattering diagram on an integral affine manifold $B, u \in B$ and $\lambda>0$. Consider an immersion

$$
\phi: S^{1} \rightarrow B \backslash \operatorname{Sing}(\mathfrak{D})^{>\lambda}
$$

in a small neighborhood of $u$ such that it intersects each ray $\mathfrak{d}$ transversally if $(\mathfrak{d}, f) \in \mathfrak{D}$ and $\left(f(u) \not \equiv \operatorname{0mod} T^{\lambda}\right)$. Assume the intersection order is $\mathfrak{d}_{1}, \cdots \mathfrak{d}_{s}$, then we form an ordered product as follows :

$$
\theta_{\gamma, \mathfrak{D}}^{u, \lambda}=\theta_{\mathfrak{d}_{1}} \circ \cdots \circ \theta_{\mathfrak{d}_{s}}
$$

where each term on right hand side is of the form

$$
\theta_{\mathfrak{d}_{i}}=\exp \left(\log \left(f_{i}(u)\right) \partial_{n_{i}}\right)
$$


with $n_{i} \in\left(\Gamma_{g}\right)^{*}$ primitive, annihilates the tangent space to $\mathfrak{d}_{i}$ and such that

$$
\left\langle n_{i}, \phi^{\prime}\left(p_{i}\right)\right\rangle>0, \text { for } p_{i} \in \operatorname{Im} \phi \cap \mathfrak{d}_{i} .
$$

Assume the special Lagrangian fibration has 24 singular fibres then the its affine structure has 24 singularities such that the monodromy around each singular point is conjugate to $\left(\begin{array}{ll}1 & 1 \\ 0 & 1\end{array}\right)$. Each singular point $v$ emanates two rays called initial rays along both monodromy invariant direction $\mathfrak{d}_{ \pm}$ with the slab function $f_{\mathfrak{d}_{ \pm}}=1+z^{m_{\mathfrak{d}_{ \pm}}} T^{\left|Z_{\gamma_{ \pm}}(u)\right|}$, where $m_{\mathfrak{d}_{ \pm}} \in \Gamma_{g}, \gamma_{ \pm} \in \Gamma$ denotes the relative classes of Lefschetz thimbles around the corresponding singularity and $Z_{\gamma_{ \pm}}=\int_{\gamma_{ \pm}} \Omega$.

The following is a modified version of statements in [23][18].

Theorem 3.4. Let $\mathfrak{D}_{\vartheta}$ be a scattering diagram given by the initial data above, then there is a scattering diagram $S\left(\mathfrak{D}_{\vartheta}\right)$ such that for any $\lambda>0$, there are only finitely many rays with nontrivial attached function modulo $T^{\lambda}$. Moreover, given $u \in B_{0}, \lambda>0$ and a closed loop $\phi$, one has

$$
\theta_{\phi, S\left(\mathfrak{D}_{\vartheta}\right)}^{u, \lambda} \equiv 0 \quad\left(\bmod T^{\lambda}\right)
$$

Proof. WLOG we may assume the phase of all central charges in the proof is zero. Notice that when $\lambda$ is small we can take $S\left(\mathfrak{D}_{\vartheta}\right)=\mathfrak{D}_{\vartheta}$. Let $\lambda_{0}$ be the smallest central charge among the intersections of the initial rays, then the statement holds for $\lambda<\lambda_{0}$. Moreover, there are only 24 initial rays. Assume the theorem holds for all $\lambda<\lambda_{k}$ and the scattering diagram $\mathfrak{D}_{\vartheta}$ only consists finitely many rays. Consider the scattering diagram contains 
only rays with central charge less than $\lambda_{k}$ at their initial points. We consider $\theta_{\rho_{p}}$ for each singularity $p$ of $\mathfrak{D}_{k}$ and a small loop $\rho_{p}$ around $p$. Note that the exponent of $T$ in $\theta_{\rho}(p)$ is discrete. Let $\lambda_{k+1}$ be the smallest exponent of $T$ appearing in $\log \left(\theta_{\rho_{p}, \mathfrak{D}_{k}}\right)$, then

$$
\theta_{\rho, \mathfrak{D}_{k}}\left(p_{0}\right) \equiv \exp \left(\sum_{i=1}^{s} c_{i} z^{\gamma_{i}} T^{\lambda_{k+1}} \partial_{n_{i}}\right) \quad\left(\bmod T^{\lambda_{k+1}+\epsilon}\right)
$$

for some singularity $p_{0}$ and $\epsilon>0$ such that $\lambda_{k+1}+\epsilon$ is less than the second small exponent of $T$ appearing in $\log \left(\theta_{\rho_{p}, \mathfrak{D}_{k}}\right)$. (Actually, there might be more than one $p_{0}$. Then we have to consider them all at the same time.) We set

$$
\left.\mathfrak{D}_{k+1}=\mathfrak{D}_{k} \cup\left\{o_{k+1}+\mathbb{R}_{\geq 0} m_{i}, 1 \pm c_{i} z^{\gamma_{i}} T^{\lambda_{k+1}}\right) \mid i=1, \cdots, s\right\}
$$

The sign is chosen such that each contribute $\exp \left(-c_{i} z^{\gamma_{i}} T^{Z_{\gamma_{i}}} \partial_{n_{i}}\right)$ to $\theta_{\rho_{p_{0}}, \mathfrak{D}_{k}}$ $\left(\bmod T^{\lambda_{k}}\right)$. The choice of $p_{0}$ guarantees that for each singularity of $p$ and a small loop $\rho_{p}$ around, we have

$$
\theta_{\rho_{p}, \mathfrak{D}_{k+1}}(p)=I d \quad\left(\bmod T^{\lambda_{k+1}}\right)
$$

By induction, it suffices to take $S\left(\mathfrak{D}_{\vartheta}\right)=\cup_{k} \mathfrak{D}_{k}$.

Definition 3.5. We will call the rays in $S\left(\mathfrak{D}_{\vartheta}\right)$ BPS rays.

Remark 3.6. It is easy to see that if we include the dependence of $\vartheta$, the exponents of each monomial of slab functions in $S\left(\mathfrak{D}_{\vartheta}\right)$ is of the form $e^{-i \vartheta} \int_{\gamma} \Omega$.

Remark 3.7. Notice that we don't have the notion of degree as in [18][23] 
therefore we need to use energy filtration instead and a static construction of the scattering diagram. At each singularity, the degree filtration and energy filtration are equivalent. The Theorem 3.4 can be viewed as a substitute of Kontsevich-Soibelman wall-crossing formula for elliptic K3 surfaces.

We will use the follow standard fact of algebra,

Lemma 3.8. Fix $\epsilon=0$ or 1 . Let $f=1+a_{1} x+a_{2} x^{2}+\cdots \in \mathbb{Q}[[x]]$, then there is a unique factorization

$$
f=\prod_{k}\left(1-(-1)^{k^{2} \epsilon} x^{k}\right)^{k d_{k}}
$$

for some $d_{k} \in \mathbb{Q}$. Moreorver, we have the estimate for size of $d_{k}$.

Lemma 3.9. Let $c(n)=\sum_{k=1} \frac{d_{n / k}}{k^{2}}$, and $d_{s}=0$ if $s$ is not an integer. Then we have

$$
\sum_{k=1}^{\infty} k d_{k} \log \left(1-(-1)^{k^{2} \epsilon} x^{k}\right)=\sum_{n=1}^{\infty} n c(n) u^{n}, \text { where } u=(-1)^{\epsilon} x
$$

Proof.

$$
\text { R.H.S. }=\sum_{k=1}^{\infty} \sum_{l=1}^{\infty} k d_{k} \frac{u^{l k}}{l}=\sum_{k, l=1}^{\infty}(k l) d_{\frac{k l}{l}} \frac{u^{l k}}{l^{2}}
$$

Set $n=k l$ we get the right hand side of the lemma.

Remark 3.10. The sign $\epsilon$ will be related to quadratic refinement in [17].

Lemma 3.11. For generic choice of $\vartheta$, the BPS rays will not pass through any singular points. An intersection of two BPS lines will not fall in the 
singularities of affine structures nor an intersection of two BPS rays is contained in more than one stability walls

Proof. For a fixed energy $\lambda \in \mathbb{N}$, there are only finitely many BPS rays and finitely many phase have BPS rays passing through singularities. Thus, the former part of the lemma simply follows from Baire's theorem. For the later part of the lemma, since intersection of two BPS lines, the discriminant locus and intersections of stability walls are at least of codimension two, and the later two are independent of the phase $\vartheta$. Notice that we might not be able to avoid the case in which more than two BPS lines intersect at the same point for generic phase.

Corollary 3.12. There is no tropical rational curves (see Definition 4.2 in the next section) for a generic $\vartheta$.

This is reasonable because generic K3 has Picard number 0 by Torelli theorem.

\subsection{Generalized Donaldson-Thomas Invariants $\Omega^{\text {trop }}$}

Now for each $\gamma \in \Gamma_{u}$, we want to define $\Omega^{\text {trop }}(\gamma ; u)$ as follow: for generic phase $\vartheta$, one can construct a scattering diagram $S\left(\mathfrak{D}_{\vartheta}\right)$ from the initial BPS rays with respect to the corresponding phase $\vartheta$. The walls of marginal stability is taken to be the closure of singularities of the scattering diagram. By straight forward computation, we have

Proposition 3.13. If the intersection product between two charges is zero, then the associate Kontsevich-Soibelman transformation commutes. In par- 
ticular, the Kontsevich-Soibelman transformation associate to a pure flavor charge is an identity.

Therefore, one can define $\Omega(\gamma ; u)$ by Lemma 3.5 and extended uniquely by requiring them to be locally constant. The Kontsevich-Soibelman wallcrossing formula follows directly from our construction.

Remark 3.14. The construction of $\Omega(\gamma)$ can be view as an inverse of the procedure in [29].

Proposition 3.15. The nontrivial $\Omega\left(\gamma_{1}+\gamma_{2}\right)$ happens on the side of the wall of marginal stability with

$$
\frac{1}{2} \frac{\left\langle\gamma_{1}, \gamma_{2}\right\rangle\left|Z\left(\gamma_{1}+\gamma_{2}\right)\right|}{\operatorname{Im}\left[Z\left(\gamma_{1}\right) \bar{Z}\left(\gamma_{2}\right)\right]}>0
$$

Proof. This is an easy consequence of the fact that central charges are holomorphic.

Proposition 3.16. Given an ellptic fibred K3 surface, the invariants $\Omega(\gamma ; u)$ are independent of choices of the Kähler class $\omega$.

Proof. For generic $\vartheta$ one can construct $S\left(\mathfrak{D}_{\vartheta}\right)$ such that there are no BPS rays passing through singularities of the affine base. The energy filtrations induced from different choices of Kähler class are equivalent. From Theorem 4.6, $\tilde{\Omega}(\gamma, u)$ depends only on the degree filtration. Together we see that the scattering diagrams $S\left(\mathfrak{D}_{\vartheta}\right)$ constructed in Theorem 3.4 is independent of the choice of the Kähler classes and thus so is the invariant contributed from $\vartheta$. 
Given an elliptic fibration K3 surface $f: X \rightarrow \mathbb{P}^{1}$ with holomorphic $(2,0)$-form $\Omega$, then any 2 -form $\alpha$ on $\mathbb{P}^{1}$ such that $\Omega^{\prime}=\Omega+f^{*} \alpha, \Omega^{\prime} \wedge \Omega^{\prime}=0$ gives rise to another elliptic fibration with same Jacobian. Moreover, any elliptic fibration with the same Jacobian arises in above construction. It is obvious that for any $\gamma \in H_{2}(X, L)$ and $\tilde{v}$ a lifting of $v \in T \mathbb{P}^{1}$,

$$
\int_{\partial \gamma} \iota_{\tilde{v}} f^{*} \alpha=0
$$

Therefore, changing elliptic fibred K3 surfaces within same Jacobian doesn’t change the affine structure and the scattering diagram. To sum up, we proved

Theorem 3.17. The invariants $\Omega^{\text {trop }}(\gamma ; u)$ only depend on the Jacobian of the elliptic fibration but are independent of the Kähler class chosen.

Conjecture 3.18. (Integrality Conjecture) $\Omega^{\text {trop }}(\gamma) \in \mathbb{Z}$, for every $\gamma \in \Gamma$.

\section{Tropical Geometry on K3 Surfaces}

\subsection{Tropical Discs}

In the previous section, we construct a scattering diagram $\mathfrak{D}=\left\{\left(\mathfrak{d}, f_{\mathfrak{d}}\right)\right\}$ on the base affine manifold from the initial data. We want to build up tropical discs from this scattering diagram formally and establish the relation between tropical counting and $\Omega(\gamma)$.

Definition 4.1. We say $B$ is a tropical affine manifold with singularities $\Delta$, if there exists an integral affine structure on $B \backslash \Delta$. 
Definition 4.2. Let $B$ be a tropical affine manifold with singularities with discriminant locus $\Delta$. Let $G$ be a weighted, connected finite graph, with its set of vertices and edges denoted by $G^{[0]}$ and $G^{[1]}$ respectively, with weight function $w_{G}: G^{[1]} \rightarrow \mathbb{N} \backslash\{0\}$. A parametrized tropical curve (with stop) in $B$ is a continuous map $\phi: G \rightarrow B$ satisfying the following conditions:

1. For every edge $E \subseteq G,\left.\phi\right|_{\text {Int }(E)}$ is an embedding, $\phi^{-1}\left(B_{0}\right)$ is dense in $\operatorname{Int}(E)$, and there is a section $u \in \Gamma\left(\operatorname{Int}(E), \phi^{*}\left(i_{*} \Lambda\right)\right)$ which is tangent to $\phi(\operatorname{Int}(E))$ at every point of $\phi(\operatorname{Int}(E)) \cap B_{0}$. We choose this section to be primitive, i.e. not an integral multiple of another section of $\phi^{*}\left(i_{*} \Lambda\right)$.

2. For every vertex $v \in G^{[0]}$, let $E_{1}, \ldots, E_{m} \in G^{[1]}$ be the edges adjacent to $v$. Let $u_{i}$ be the section of $\left.\phi^{*}\left(i_{*} \Lambda\right)\right|_{\operatorname{Int}\left(E_{i}\right)}$ promised by (1), chosen to point away from $v$. This defines germs $u_{i} \in \phi^{*}\left(i_{*} \Lambda\right)_{v}=\left(i_{*} \Lambda\right)_{\phi(v)}$.

(a) If $\phi(v) \in B_{0}$, the following balancing condition holds in $\Lambda_{\phi(v)}$ except at the stop $\phi(v)$ :

$$
\sum_{j=1}^{m} w_{G}\left(E_{j}\right) u_{j}=0 .
$$

(b) If $\phi(v) \notin B_{0}$, then all the edge attached to vertex $v$ should be mapped to the monodromy invariant direction.

3. If $\phi$ is a tropical rational curve with stop at $p \in B_{0}$, then we give each edge an orientation that it points from the vertex with larger distance to $p$ to the vertex with smaller distance to $p$. In particular, every vertex has a unique outgoing edge and others are ingoing edges. 
We will also call tropical curves with stop by tropical discs. The balancing condition will make the following definition well-defined.

Definition 4.3. Let $\phi: G \rightarrow B$ be a parametrized tropical curves with only trivalent vertices. The multiplicity at a vertex $V \in G^{[0]}$ is

$$
\operatorname{Mult}_{V}(\phi)=w_{1} w_{2}\left|m_{1} \wedge m_{2}\right|
$$

where $E_{1}, E_{2}$ are two of the edge containing $V$ and $w_{i}=w_{\Gamma}\left(E_{i}\right)$ and $m_{i} \in M$ is the primitive integral vector in the direction of $\phi\left(E_{i}\right)$.

Definition 4.4. 1. Let $h$ be a tropical curve (with stop) has only trivalent interior vertices. The multiplicity of a tropical curve (with stop) $\phi$ is defined by

$$
\operatorname{Mult}(h)=\prod_{V \in G^{[0]}} \operatorname{Mult}_{V}(\phi)
$$

2. Given directions (might repeated) $m_{i} \in M, N^{\text {trop }}(\mathbf{w})$ is the weighted count of the number of tropical curves (with stop) has in the directions $m_{i}$ with multiplicities $w_{i}$ defined above.

\subsection{Tropical Discs Counting, $\Omega^{\text {trop }}$ and Wall-Crossing}

Definition 4.5. (Central charge of tropical discs)Let $(X, \omega, \Omega)$ be an elliptic K3 surfaces. Given a tropical discs $\phi$ with stop at $u$ on an tropical affine manifold B induced from the affine coordinate of special Lagrangian fibration on $X_{\vartheta}$, we will associate it with a central charge as follows by induction on the number of singularities of affine structure $\phi$ hits: If the $\phi$ only hits only one singularity, then let $[\phi] \in H_{2}\left(X, L_{u}\right)$ be the relative class of Lefschetz 
thimble such that $\int_{[\phi]} \omega_{\vartheta}>0$ and its central charge $Z_{\phi}=\int_{[\phi]} \Omega$. Assume $p$ is an internal vertex of $\phi$ and each let $\phi_{1}, \cdots \phi_{s}$ are the components of $\operatorname{Im}(\phi) \backslash p$ containing an ingoing edge of $p$. By induction we already define $\left[\phi_{i}\right] \in H_{2}\left(X, L_{p}\right)$ and the corresponding central charges $Z_{\phi_{i}}=\int_{\left[\phi_{i}\right]} \Omega$ for each $i=1, \cdots, s$. For any $p^{\prime}$ on the outgoing edge of $p$, there is a natural tropical disc $\phi^{\prime}$ with stop at $p^{\prime}$ induced from $\phi$, then we define $\left[\phi^{\prime}\right] \in H_{2}\left(X, L_{p^{\prime}}\right)$ the parallel transport of $\sum_{i=1}^{s}\left[\phi_{i}\right]$ along the outgoing edge of $p$ to $p^{\prime}$. The central charge of $\phi^{\prime}$ is given by $Z_{\phi^{\prime}}=\int_{\left[\phi^{\prime}\right]} \Omega$.

The following theorem is a modification of Theorem 2.8 [16].

Theorem 4.6. When $u$ cross a wall consisting of relative classes $\gamma_{i}, i=$ $1, \cdots, n$, then one has the following wall-crossing formula for $\tilde{\Omega}^{\text {trop }}$ :

$$
\Delta \tilde{\Omega}^{\text {trop }}(d \gamma)=\sum_{\mathbf{w}: \sum\left|\mathbf{w}_{\mathbf{i}}\right| \gamma_{i}=d \gamma} \frac{N^{\text {trop }}(\mathbf{w})}{|A u t(\mathbf{w})|}\left(\prod_{1 \leq i \leq n, 1 \leq j \leq l_{i}} \tilde{\Omega}^{\text {trop }}\left(w_{i j} \gamma_{i}\right)\right)
$$

where $\mathbf{w}=\left(\mathbf{w}_{1}, \cdots, \mathbf{w}_{n}\right), \mathbf{w}_{i}=\left(w_{i 1}, \cdots, w_{i l_{i}}\right) \in \mathbb{Z}_{\geq 0}^{l_{i}}$, and $\left|\mathbf{w}_{i}\right|=\sum_{k=1}^{l_{i}} w_{i k}$. Proof. To compute $\tilde{\Omega}^{\text {trop }}(d \gamma)$ for a fixed $d$, it suffices to compute the attached function $f_{\mathfrak{d}}$ (modulo $T^{Z_{\gamma}+\epsilon}$ ) of the BPS ray $\mathfrak{d}$ in the scattering diagram $\mathfrak{D}$ associate $\gamma_{i}$ with a generic phase construct in theorem 3.4 , where $\mathfrak{d}$ is the direction given by $\gamma$.

Replacing $T^{Z_{\gamma_{i}}}$ by $t_{i}$ in $\mathfrak{D}$, we get a compatible scattering diagram $\mathfrak{D}^{\prime}=$ $\left\{\left(\mathfrak{d}_{i}, f_{i}\right) \mid i=1, \cdots, n\right\}$ over $\mathbb{C}\left[\left[t_{1}, \cdots, t_{n}\right]\right]$, where $\mathfrak{d}_{i}=\mathbb{R} m_{i}$ and

$$
\log f_{\mathfrak{D}_{i}}=\sum_{j=1}^{k} \sum_{w \geq 1} w a_{i w} z^{w m_{i}} t_{i}^{w} \in R_{k}=\frac{\mathbb{C}\left[\left[t_{1}, \cdots, t_{n}\right]\right]}{\left(t_{1}^{k+1}, \cdots, t_{n}^{k+1}\right)}
$$


is Taylor expansion in $t_{i}$, with $a_{i j w} \in \mathbb{C}$. Since energy filtration is equivalent to degree filtration, it suffices to compute $f_{\mathfrak{d}} \in S\left(\mathfrak{D}^{\prime}\right)$.

We follow the trick in [16] to make the substitution $t_{i}=\sum_{j=1}^{k} u_{i j}$ and then do the expansion.

$$
\log f_{\mathfrak{d}}=\sum_{w=1}^{k} \sum_{\# J=j} j ! w a_{i w} z^{w m_{i}} \prod_{l \in J} u_{i l}
$$

Note that each $u_{i j}$ squares to zero and now $f_{i}$ can be split into simple forms,

$$
f_{\mathfrak{o}}=\prod_{j=1}^{k} \prod_{\# J=j}\left(1+j ! w a_{i w} z^{w m_{i}} \prod_{l \in J} u_{i l}\right)
$$

Each ray $\mathfrak{d} \in S\left(\mathfrak{D}^{\prime}\right)$ will correspond to a trivalent tree and

$$
f_{\mathfrak{o}}=1+w_{\text {out }} \operatorname{Mult}(h) \prod_{i, w, \# J=w}\left(w ! a_{i w} \prod_{j \in J} u_{i j}\right) z^{m_{\text {out }}}
$$

where the $i, J, w$ run through all indices such that $m_{\text {out }}=w_{\text {out }} m_{\text {out }}^{\prime}, m_{\text {out }}^{\prime}$ primitive. For large enough $k$ and summing them up gives

$$
\log f_{\mathfrak{d}}=\sum_{\mathbf{w}: \sum\left|\mathbf{w}_{i}\right| m_{i}=d \gamma} d \frac{N^{\text {trop }}(\mathbf{w})}{|A u t(\mathbf{w})|}\left(\prod_{1 \leq i \leq n, 1 \leq j \leq l_{i}} a_{i w_{i j}} t_{i}^{w_{i j}}\right) z^{\sum_{i}\left|\mathbf{w}_{i}\right| m_{i}}
$$

Plugging in $t_{i}=T^{Z_{\gamma_{i}}}$, and by induction we have $a_{i w_{i j}}=\tilde{\Omega}^{\text {trop }}\left(w_{i j} \gamma_{i}\right)$. Comparing the coefficients, we get the wall crossing formula for $\tilde{\Omega}^{\text {trop }}$

Example 4.7. Assume there are two BPS rays hitting at $p$ from direction $(1,0)$ and $(0,1)$. 
1. To compute $\tilde{\Omega}^{\text {trop }}(1,2)=0$,

$$
\begin{aligned}
(1,2) & =1 \cdot(1,0)+2 \cdot(0,1) \Rightarrow N^{\text {trop }}=1, \text { Aut }=2 \rightsquigarrow \frac{1 \cdot 1}{2} \cdot 1 \cdot 1^{2}=\frac{1}{2} \\
& =1 \cdot(1,0)+1 \cdot(0,2) \Rightarrow N^{\text {trop }}=1, \text { Aut }=1 \rightsquigarrow \frac{1 \cdot 2}{1} \cdot \frac{-1}{2^{2}}=\frac{-1}{2}
\end{aligned}
$$

2. To compute $\tilde{\Omega}^{\text {trop }}(2,2)=0$,

$$
\begin{aligned}
(1,2) & =1 \cdot(2,0)+1 \cdot(0,2) \Rightarrow N^{\text {trop }}=4, \text { Aut }=1 \rightsquigarrow \frac{2 \cdot 4}{1} \cdot \frac{-1}{2^{2}} \frac{-1}{2^{2}}=\frac{1}{2} \\
& =1 \cdot(2,0)+2 \cdot(0,1) \Rightarrow N^{\text {trop }}=4, \text { Aut }=2 \rightsquigarrow \frac{2 \cdot 4}{2} \cdot \frac{-1}{2^{2}} \cdot 1^{2}=-1 \\
& =2 \cdot(1,0)+1 \cdot(0,2) \Rightarrow \cdots=-1 \\
& =2 \cdot(1,0)+2 \cdot(0,1) \Rightarrow N^{\text {trop }}=2, \text { Aut }=2^{2} \rightsquigarrow \frac{2 \cdot 4}{2^{2}} \cdot 1^{2} \cdot 1^{2}=1
\end{aligned}
$$

Together with the data from initial rays,

$$
\tilde{\Omega}^{t r o p}\left(d \gamma_{e}\right)=\frac{(-1)^{d-1}}{d^{2}}
$$

where $\gamma_{e}$ is the Lefschetz thimble from each singularity. One achieves the relation between tropical counting and $\left\{\tilde{\Omega}^{\text {trop }}\right\}$. Therefore,

Theorem 4.8. The true tropical count is sum of product of tropical counts in (15) at each vertex after infinitesimal deformation. Moreover, these tropical discs with nontrivial tropical invariants of an elliptic K3 correspond to the instanton corrections (in the sense of [17]) of complex structure of its mirror.

Proof. The first statement follows directly from the construction of the tropical invariants and (15) by induction on the number of internal vertices. For 
the second statement, it suffices to prove it for Ooguri-Vafa space [4], since all the rest of the tropical discs/walls of instanton corrections are generated by wall-crossing. From remark 2.11, for a fixed $\zeta$ torus fibre $T_{y}$ bounds holomorphic discs if $\operatorname{Re}(y \bar{\zeta})=0$ while the wall of instantons are given by $\left\{y \in B_{0} \mid \operatorname{Im}(y \bar{\zeta})=0\right\}$.

Remark 4.9. In physics literature, the mass $M$ of any charge $\gamma$ obeys

$$
M \geq\left|Z_{\gamma}\right|
$$

where the mass is $\int_{\gamma}|\Omega|$ along a path. We call the charge $\gamma$ is BPS if and only if the equality holds Thus, a charge is BPS if only if its phase of central charge is the same angle along the path. Therefore, it is reasonable to expect tropical discs to correspond to BPS charges.

Remark 4.10. The form of (15) is similar to wall-crossing formula of Joyce-Song. The $\mathbf{w}$ in (15) might related to the spin by $j=\sum\left(w_{i j}-1\right)$ in the refined wall-crossing formula and leads to refined tropical invariants.

\section{Tropical versus Holomorphic}

The classical way of constructing tropical discs is taking certain adiabatic limit of the image of the holomorphic discs under the fibration. However, this method usually involves hard analysis and we don't know much about the Calabi-Yau metric. Therefore, we introduce here another point of view of tropical discs from the locus of Lagrangian fibres bounding the prescribed class of holomorphic discs. 
Observation 5.1. From Remark 2.3, the set of special Lagrangian torus fibres bounding holomorphic discs of a same relative class fall above a hyperplane on the base affine manifolds.

Similar to $\Omega^{\text {trop }}$, we have support property [24] for holomorphic discs because holomorphic cycles are calibrated . Namely,

Proposition 5.2. There exists $\delta>0$, such that

$$
\frac{\left|Z_{\gamma}\right|}{\|\gamma\|}>\delta
$$

for all $\gamma \in \bigcup_{\vartheta \in S^{1}} H_{2}^{\prime}\left(X_{\vartheta}, L\right)$.

Proof. We first choose $\left\{\left(\alpha_{i}, \beta_{i}\right)\right\} \in H^{2}\left(X_{\vartheta}, L\right)$ as basis with $\alpha_{i} \in \mathcal{A}^{2}\left(X_{\vartheta}\right.$, $\beta_{i} \in \mathcal{A}^{1}(L)$ such that $d \alpha_{i}=0$ and $\left.\alpha_{i}\right|_{L}=d \beta_{i}$. Then the non-degenerate pairing is given by

$$
\begin{aligned}
H_{2}\left(X_{\vartheta}, L\right) \times H^{2}\left(X_{\vartheta}, L\right) & \longrightarrow \mathbb{R} \\
\left(\gamma, \quad\left(\alpha_{i}, \beta_{i}\right)\right) & \mapsto \int_{\gamma} \alpha_{i}-\int_{\partial \gamma} \beta_{i}=\int_{\gamma} \alpha_{i}-d \tilde{\beta}_{i},
\end{aligned}
$$

where $\tilde{\beta}_{i}$ are fixed smooth extension of $\beta_{i}$ to whole $X_{\vartheta}$. Then if $\gamma$ can be represented by a holomorphic cycle in $X_{\vartheta}$, we have

$$
\left|\int_{\gamma}\left(\alpha_{i}, \beta_{i}\right)\right| \leq \operatorname{Vol}(\gamma) \cdot \sup _{\substack{v_{1}, v_{2} \in T_{p} X,\left|v_{1} \wedge v_{2}\right|=1}}\left\langle\alpha_{i}-d \tilde{\beta}_{i}, v_{1} \wedge v_{2}\right\rangle \leq C_{\vartheta} \cdot\left|Z_{\gamma}\right|
$$

Summing $i$ through basis of $H^{2}\left(X_{\vartheta}, L\right)$, the left hand side gives a norm on $H_{2}\left(X_{\vartheta}, L\right)$ and prove the support property for a fixed $\vartheta$. The proposition 
follows immediately because $C_{\vartheta}$ is continuous depending on $\vartheta \in S^{1}$ and $S^{1}$ is compact.

On one hand, the support property is required to define stability data for a suitable category. On the other hand, it might be needed to prove the convergence of hyperKäher metric in [17] though we don't need it here.

Definition 5.3. Given local section $\gamma$ of $\bigcup_{u} H_{2}\left(X, L_{u}\right)$, we define locally

$W_{\gamma_{1}, \gamma_{2}}^{\prime \prime}=\left\{u \in B_{0} \mid \operatorname{Arg} Z_{\gamma_{1}}=A \operatorname{Arg} Z_{\gamma_{2}}, Z_{\gamma_{1}} Z_{\gamma_{2}} \neq 0\right.$ and $\gamma_{1}, \gamma_{2}$ are not colinear $\}$, and $W_{\gamma}^{\prime \prime}=\bigcup_{\gamma_{1}+\gamma_{2}} W_{\gamma_{1}, \gamma_{2}}^{\prime \prime}$. We say a charge $\gamma \in H_{2}\left(X, L_{u}\right)$ is primitive if $u \notin W_{\gamma}$ and $\gamma$ is not divisible by an integer.

Because the central charge $Z_{\gamma}$ is holomorphic, each $W_{\gamma_{1}, \gamma_{2}}^{\prime \prime}$ forms a real analytic Zariski closed subset of real codimension one on $B_{0}$. Indeed, let $f$ be the defining equation of $W_{\gamma_{1}, \gamma_{2}}^{\prime \prime}$ and $f+i g$ is holomorphic on a domain of $\mathbb{C}^{2}$. By Cauchy-Riemann equation $f$ will not have accumulate critical points. At every isolated critical point $u$ of $f$, we first write

$$
f+i g=w^{n} \text {, where } n \text { is the zero order of } f+i g \text { at } u \text {, }
$$

and $w$ is an holomorphic function of $z$ and locally invertible. Therefore, $f=0$ is characterized by $n$ real codimension one smooth hypersurfaces intersecting at $u$. Notice that the sublattice which is the preimage of $\mathbb{R} e^{i \vartheta}$ is a sub $\mathbb{Z}$-module and thus a sublattice. In particular, the union in the definition of $W_{\gamma}^{\prime \prime}$ is finite. Therefore, $W_{\gamma}^{\prime \prime}$ is locally a real analytic Zariski open subset of the base by maximal principle unless multiple cover occurs 
(which is excluded by definition). If a relative class $\gamma \in H_{2}(X, L)$ can be represented as a holomorphic cycle, the phase of central charge $\int_{\gamma} \Omega$ will indicate which complex structure $J_{\vartheta}$ makes $\gamma$ holomorphic. This motivate the following motivation:

Definition 5.4. Given local section $\gamma$ of $\Gamma=\bigcup_{u} H_{2}\left(X, L_{u}\right)$, we define locally

$$
\begin{aligned}
W_{\gamma}^{\prime} & =\bigcup_{\gamma_{1}+\gamma_{2}=\gamma} W_{\gamma_{1}, \gamma_{2}}^{\prime} \\
& =\left\{u \in B \mid \gamma=\gamma_{1}+\gamma_{2} \text {, where } \gamma_{1} \text { and } \gamma_{2}\right. \text { are represented by holomorphic }
\end{aligned}
$$
discs with boundary on $L_{u}$ in $X_{\vartheta}$ and $\gamma_{1}, \gamma_{2}$ are not colinear $\}$

Notice that by Gromov compactness theorem (corollary 5.5 [10]) we have $W_{\gamma_{1}, \gamma_{2}}^{\prime} \subseteq W_{\gamma_{1}, \gamma_{2}}^{\prime \prime}$ as a closed subset in standard topology on $B_{0}$ and the expression in (19) is a finite union. However, $W_{\gamma}^{\prime}$ might not be real codimension one because of appearance of holomorphic discs with respect to non-generic (almost) complex structures. Also, $W_{\gamma_{1}, \gamma_{2}}^{\prime}$ might depend on the choice of Ricci-flat $\omega$ while the real codimension one $W_{\gamma_{1}, \gamma_{2}}^{\prime \prime}$ are not.

Remark 5.5. Changing the special Lagrangian boundary conditions is expected to be mirror to changing the stability conditions of stability condition in Donaldson-Thomas theory. Since our central charge is constraint by $\langle d Z, d Z\rangle=0$, the affine base $B$ can be viewed as a complex isotropic submanifold of the corresponding stability manifold. 


\subsection{Disc Contribution from Local}

On Ooguri-Vafa space, there is a unique holomorphic disc with boundary on every special Lagrangian torus over monodromy invariant direction. Thus it gives raise to a special Lagrangian disc after hyperKähler rotation. To prove there exists such a holomorphic discs near the $I_{1}$-type singular fibre of K3 surface with special Lagrangian, we need some understanding of geometry of K3 surfaces near large complex limit. Gross and Wilson construct an approximate metric by gluing Ooguri-Vafa metric with the semi-flat metric for elliptic K3 surfaces when the Kähler class goes large (along a straight line in Kähler cone). Moreover, they derive some estimate for the approximate metric:

Theorem 5.6. ([19] Gross-Wilson ) Let $X$ be an elliptic K3 surface with holomorphic volume form $\Omega$. There exists $\omega_{\epsilon}$ the approximate metric equal to the twisted Ooguri-Vafa metric near the singular fibres and semi-flat metric [20] away from singular fibres and with fibre size $\epsilon$. Moreover, if $F_{\epsilon}=$ $\log \left(\frac{\Omega \wedge \Omega^{2} / 2}{\omega_{\epsilon}^{2}}\right)$, then the solution $u_{\epsilon}$ of the Monge-Ampere equation

$$
\operatorname{det}\left(g_{i j}+i \partial_{i} \bar{\partial}_{\bar{j}} u_{\epsilon}\right)=e^{F_{\epsilon}}
$$

satisfies the follow exponential decay property

$$
\left\|u_{\epsilon}\right\|_{C^{0}} \leq D_{1} e^{-D_{2} / \epsilon}
$$

for some positive constant $D_{1}, D_{2}$.

We will use this asymptotic behavior of metric as the working definition 
of large complex limits.

Heuristically, Gross-Wilson proves that the Ricci-flat metric converges to semi-flat metric when the K3 surface goes to the large complex limit. However, this does not include even the instaton correction contribute from the initial rays.

Assume $y$ is on the BPS ray emanated from a singular point. Take a neighborhood $\mathcal{U}$ of this segment from the singularity to $y$ and lift the special Lagrangian fibration to the universal cover of $\mathcal{U}$. The affine coordinate on $\mathcal{U}$ is $\left(y_{1},-\operatorname{Im} \int \tau d y\right)$ when $|y|<\epsilon$ or $|y|>\epsilon$. Here we assume the monodromy invariant direction is characterized by $y_{1}=0$. Similar to the case near the singular point, to prove that the fiber $T_{y}$ can bound a holomorphic disc corresponding to the initial BPS ray, it suffices to construct an approximate solution with respect to the approximate metric constructed by Gross-Wilson. From [19], there exist a holomophic section $\sigma$ and a real function $\phi$ such that

$$
\omega_{S F}-T_{\sigma}^{*} \omega_{O V}=i \partial \bar{\partial} \phi
$$

Note that the local $S^{1}$-action still live on the torus fibration over $\mathcal{U}$, therefore, we can average $\phi$ with respect to the local $S^{1}$-action and assume it is $S^{1}$ invariant.

$$
\begin{aligned}
\omega_{\text {new }}= & \omega_{S F}+i \partial \bar{\partial}\left(\psi\left(|y|^{2}\right) \psi\right) \\
= & \left(1-\psi\left(|y|^{2}\right)\right) \omega_{S F}+\psi\left(|y|^{2}\right) T_{\sigma}^{*} \omega_{O V} \\
& -i\left(\psi^{\prime}\left(|y|^{2}\right) \bar{y} d y \wedge \bar{\partial} \phi+\psi^{\prime}\left(|y|^{2}\right) y \partial \phi \wedge d \bar{y}+\psi^{\prime \prime}\left(|y|^{2}\right)|y|^{2} \phi d y \wedge d \bar{y}\right) .
\end{aligned}
$$


Remark 5.7. There is actually another term should be added to $\omega_{\text {new }}$ to make it in the right cohomology class.

The candidate for the approximate holomorphic disc is given by $y_{1}=$ $u=0$, so we want to prove that $(\omega)_{\text {new }}$ restricted to zero. It is easy to see that the first, second and last term restrict to zero. Then third and fourth term together restrict to the disc is $-2 y_{2} d y_{2} \wedge \operatorname{Re} \partial \phi=-y_{2} d y_{2} \wedge d \phi$ but $d \phi\left(\frac{\partial}{\partial t}\right)=0$ since $\phi$ is real and $S^{1}$-invariant. Thus, we get a smooth special Lagrangian discs with respect to approximate metric $(\omega)_{\text {new }}$ and boundary on an elliptic torus fibre.

The following proof is the standard deformation theory of special Lagrangian submanifolds (with boundaries) modified from [1]. Start with a smooth special Lagrangian disc $f:\left(D^{2}, \partial D^{2}\right) \rightarrow\left(\left(X, \omega_{a}, \Omega\right), L\right)$ we consider the a family of embeddings

$$
\begin{aligned}
\Phi_{V}:\left(D^{2}, \partial D^{2}\right) & \rightarrow\left(\left(X, \omega_{t}, \Omega_{t}\right), L\right) \\
\phi_{V}(x) & =\exp _{f(x)}(V(x)),
\end{aligned}
$$

where $\omega_{t}=\omega_{a}+t\left(\omega-\omega_{a}\right), \Omega_{t}=e^{i \theta(t)} \Omega$. Also, exp is specially constructed and $V$ should satisfy the Neumann boundary condition to keep $\Phi_{V}\left(\partial D^{2}\right) \subseteq L$.

It is natural to write down

$$
\begin{array}{r}
F(V . t): W^{1, p}\left(N_{L / X}\right) \times \mathbb{R} \rightarrow X \\
F(V, t)(x)=\left(\left(\exp _{x} V(x)\right)^{*} \omega_{t},\left(\exp _{x} V(x)\right)^{*} \operatorname{Im}\left(e^{i \theta(t)} \Omega_{t}\right)\right)
\end{array}
$$

The embedding $\Phi_{V}\left(D^{2}, \partial D^{2}\right)$ gives a special Lagrangian disc on $\mathrm{K} 3(X, \omega, \Omega)$ 
if and only if $F(V, 1)=0$.

To solve the equation, we first compute it's linearization.

$$
d F_{(V, t)}(W, s)=(\exp V)^{*}\left(d \iota_{V} \omega_{t}+\left.s \frac{d \omega_{u}}{d u}\right|_{t}, d \iota_{V} \operatorname{Im} \Omega_{t}+\left.s \frac{d \operatorname{Im}\left(e^{i \theta(u)} \Omega_{u}\right)}{d u}\right|_{t}\right)
$$

In particular, the linearization at $(0,0)$

$$
d F_{(0,0)}(W, s)=\left(d \eta+\left.s \frac{d \omega_{t}}{d t}\right|_{0}, d *(\psi \eta)+s \psi \theta^{\prime}(0) \operatorname{vol}_{g_{a}}\right)
$$

where $\eta=\iota_{W} \omega_{a}$ is a 1 -form and $\left.\operatorname{Re} \Omega\right|_{D^{2}}=\psi v o l_{g_{a}}$. Notice that $*(\psi \eta)=*_{\psi} \eta$, where $*_{\psi}$ is the Hodge star operator for the another metric $g_{\psi}$.

Note that $F$ will factor through $C^{0, \beta}\left(d \Omega^{1}\left(D^{2}\right)\right) \times C^{0, \beta}\left(d \Omega^{3}\left(D^{2}\right)\right)$ because $\left[(\exp V)^{*} \omega_{t}\right]=\left[\omega_{a}\right]=0$ and $\left[(\exp V)^{*} \operatorname{Im} \Omega_{t}\right]=[\operatorname{Im} \Omega]=0$.

Proposition 5.8. The linearized operator

$$
d F_{(0,0)}: C^{1, \beta}\left(N_{L / X}\right)^{N} \times \mathbb{R} \rightarrow C^{0, \beta}\left(d \Omega^{1}\left(D^{2}\right)\right) \times C^{0, \beta}\left(d \Omega^{1}\left(D^{2}\right)\right)
$$

is surjective, if $\theta(t)$ chosen suitably.

Proof. Let $N$ be a normal vector field of $\partial D^{2}$ and $\alpha \in C^{1, \beta}\left(\Omega^{1}\left(D^{2}\right)\right.$ and $\beta \in C^{1, \beta}\left(\Omega^{1}\left(D^{2}\right)\right)$. We are trying to solve the system

$$
\begin{array}{r}
d \eta=d \alpha+\left.\frac{d \omega_{t}}{d t}\right|_{0} \\
d *_{\psi} \eta=d \beta+\left.\frac{d \operatorname{Im} \Omega_{t}}{d t}\right|_{0} \\
\eta(N)=0
\end{array}
$$


Hodge theory for a manifolds with boundary (p.123 [35]) shows that this system of equations can be solved with Holder regularity if and only if

1. $d\left(d \alpha+\left.\frac{d \omega_{t}}{d t}\right|_{0}\right)=0=d\left(d \beta+\left.\frac{d \operatorname{Im} \Omega_{t}}{d t}\right|_{0}\right)$

2. $\left.\left(d \beta+\left.\frac{d \operatorname{Im} \Omega_{t}}{d t}\right|_{0}\right)\left(E_{1}, E_{2}\right)\right|_{\partial D^{2}}=0$ for any vector $E_{1}, E_{2}$ tagent to $\partial D^{2}$.

3. $\int_{D^{2}}\left(d \alpha+\left.\frac{d \omega_{t}}{d t}\right|_{0}\right) *_{\psi} \lambda=0$, for every $\psi$-harmonic form $\lambda$ of $D^{2}$ satisfying Neumann boundary condition.

4. $\int_{D^{2}} *_{\psi}\left(d \beta+\left.\frac{d \operatorname{Im} \Omega_{t}}{d t}\right|_{0}\right) *_{\psi} \kappa=0$, for every $\psi$-harmonic form $\kappa$ of $D^{2}$ satisfying Neumann boundary condition.

Notice that result quoted in [35] are stated for differential forms with Sobolev regularity but extend to Hölder regularity by standard bootstrapping argument for elliptic operators. Therefore, $d F$ is surjective if there is no cohomological obstruction from $\left(\omega_{t}, \operatorname{Im}\left(e^{i \theta(t)} \Omega_{t}\right)\right)$, which can be achieved if we choose $\theta(t)$ such that

$$
\int_{D^{2}} d \beta+\theta^{\prime}(0) \int_{D^{2}} \psi \operatorname{Vol}_{g_{a}}=0
$$

For the injectivity of $d F_{(0,0)}$, consider the following system of equation

$$
\begin{aligned}
d \eta+s\left(\omega-\omega_{a}\right) & =0 \\
d *_{\psi} \eta+s \psi \theta^{\prime}(0) \operatorname{vol}_{g_{a}} & =0 \\
\eta(N) & =0
\end{aligned}
$$


Integrate the second equation over the disc $D^{2}$, we have

$$
\begin{aligned}
s \psi \theta^{\prime}(0) \operatorname{Vol}\left(D^{2}\right) & =-\int_{D^{2}} d *_{\psi} \eta \\
& =-\int_{\partial D^{2}} *_{\psi} \eta=0
\end{aligned}
$$

The last equality holds because $\mathbf{t}(* \eta)=*(\mathbf{n} \eta)=0$ (proposition 1.2.6 [35]). Thus the solutions of the system are exactly harmonic 1-forms with Neumann boundary condition on $D^{2}$ (section 6 [35]), which has the same dimension as $b^{1}\left(D^{2}\right)=0$. However, the distance from Ooguri-Vafa space to a neighborhood of $I_{1}$-type singular fibre of K3 surface is non-trivial. Therefore, we need the following quantitative implicit function theorem.

Proposition 5.9. Assume that $\mathcal{B}_{1}, \mathcal{B}_{2}$ are Banach spaces. $F: \mathcal{B}_{1} \times \mathbb{R} \rightarrow \mathcal{B}_{2}$ is a map with continuous Frechet derivative. If we have

1. $\frac{\partial F}{\partial V}(0,0): \mathcal{B}_{1} \rightarrow \mathcal{B}_{2}$ is invertible and $\left\|\frac{\partial F}{\partial V}(0,0)^{-1}\right\| \leq C$.

2. There exists $r_{0}>r>0, t_{0}>0$ such that for every $(V, t) \in U_{\mathcal{B}_{1}}\left(r_{0}\right) \times$ $\left[0, t_{0}\right]$

$$
\left\|\frac{\partial F}{\partial V}(V, t)-\frac{\partial F}{\partial V}(0,0)\right\| \leq \frac{1}{2 C}, \quad\|F(0, t)\|_{\mathcal{B}_{1}} \leq \frac{r}{2 C} .
$$

Then there exists a unique $C^{1}$-path $V(t)$ in $U_{\mathcal{B}_{1}}(r)$ for each $t \in\left[0, t_{0}\right]$ such that $F(V(t), t)=0$.

To apply proposition 5.9 , we still need an effective bound for $\left\|\frac{\partial F}{\partial V}(0,0)^{-1}\right\|$ and $\left\|\frac{\partial F}{\partial V}(V, t)-\frac{\partial F}{\partial V}(0,0)\right\|$. 
The former is has estimate

$$
\left\|\frac{\partial F}{\partial V}(0,0)^{-1}\right\| \leq C \lambda_{1} \leq \frac{C^{\prime}}{\operatorname{diam}(X)^{2}}=O(\epsilon)
$$

the first inequality is first eigenvalue estimate and the second inequality from [19]. For the later one, we have $\left\|\frac{\partial F}{\partial V}(V, t)-\frac{\partial F}{\partial V}(0,0)\right\|,\|F(0, t)\|_{\mathcal{B}_{1}}$ are of order $O\left(C_{1} e^{-D_{2} / \epsilon}\right)$ from estimate in [19].

To sum up, we proved

Theorem 5.10. Assume $X$ is a K3 surfaces with special Lagrangian fibration and 24 nodal singular fibres near large complex limit. Then around each singular fibre, each torus fibre sits above the initial ray bound a holomorphic disc in the relative class same as Lefschetz thimble with respect to the one of the complex structure in the $S_{\vartheta}^{1}$-family. For any $y$ over the initial BPS ray does not pass through any singular point in an non-monodromy invariant direction, $T_{y}$ bounds an immersed holomorphic disc when $X$ is close enough to the large complex limit.

We will call this disc as initial disc from now on.

There is another way to prove that the regularity of the holomorphic discs by applying the automatic transversality for K3 surfaces [37]

Proposition 5.11. Assume $X$ is a $K 3$ surface and $L$ is a special Lagrangian, then the bundle pair $(T X, T L)$ always has Maslov index is 0 . Let $f:\left(D^{2}, \partial D^{2}\right) \rightarrow(X, L)$ to be a holomorphic disc with boundary on $L$. Let $\mathbf{D}_{f}^{N}$ and $\mathbf{D}_{f}^{T}$ be the normal and tangent splitting of the usual linearized Cauchy-Riemann operator of $f$, then 
1. $\mathbf{D}_{f}^{N}$ is injective.

2. $f$ is regular (in the sense of next section) if and only if it is immersed or equivalently, $\left(f^{*} T X, f^{*} T L\right)_{\mathbb{C}} \cong \mathcal{O}_{\mathbb{P}^{1}}(-2) \oplus \mathcal{O}_{\mathbb{P}^{1}}(2)$.

Proof. The first part is follows from the fact that $\operatorname{ind}\left(\mathbf{D}_{f}^{N}\right)$ is less than zero and by proposition 2.2 (i) [37]. The second part is a consequence of theorem 3 in $[37]$

Remark 5.12. The author do not know any direct modification of the proof of automatic transversality for $S^{1}$-family due to the fact that the line bundle generated by $K_{J}(f, \alpha)$ doesn't have a good splitting with respect to $\mathbf{D}^{N}$ and $\mathbf{D}^{T}$.

\section{$5.2 \quad J$-Holomoprhic Discs in the $S^{1}$-Family}

Let $(X, \omega, \Omega)$ be a hyperKähler manifold (not necessarily compact) with Kähler form $\omega$ and holomorphic symplectic 2-form $\Omega$, then the hyperKähler triple $(\omega, \Omega)$ will gives a twistor $\mathbb{P}^{1}$. Let $L$ be a holomorphic Lagrangian with respect to $\Omega$, then there is $S^{1}$-family of complex structure in the twistor family such that $L$ is special Lagrangian. We denote $\mathfrak{X} \rightarrow S^{1}$ to be the family contains $L$ as special Lagrangian submanifold.

Let $\mathcal{M}_{k+1, \beta}(\mathfrak{X}, L)$ be the moduli space of pesudo-holomorphic disc in the family with boundary on the fixed special Lagrangian $L$ with $k+1$ boundary marked points in counter-clockwise order.

Let $f: \Sigma \rightarrow X_{\vartheta} \in \mathcal{M}_{0, \beta}(\mathfrak{X}, L)$. For each $\alpha \in \mathbb{R} \operatorname{Im} \Omega_{\vartheta} \subseteq\left(\Omega_{\vartheta}^{2,0} \oplus \Omega_{\vartheta}^{0,2}\right)_{\mathbb{R}}$, 
we associate an endomorphism $K_{\alpha}: T X \rightarrow T X$ by

$$
g\left(u, K_{\alpha} v\right)=\alpha(u, v)
$$

and we consider the twisted $\bar{\partial}$-equation

$$
\bar{\partial}_{J} f=K_{J}(f, \alpha)=\frac{1}{2} K_{\alpha}(\partial f \circ j)
$$

where $J=J_{\vartheta}$. The map satisfies the above twisted $\bar{\partial}$-equation are called $(J, \alpha)$-holomorphic. One can shows that $(J, \alpha)$-holomorphic maps are indeed $J_{\alpha}$-holomophic with

$$
J_{\alpha}=\frac{1-|\alpha|^{2}}{1+|\alpha|^{2}} J-\frac{2}{1+|\alpha|^{2}} K_{\alpha}
$$

in the twistor $S^{1} \subseteq \mathbb{P}^{1}$ making $C$ special Lagrangian.

Proposition 5.13. [26] Let $j$ be the complex structure on $\Sigma$. Let $g$ be in the conformal class of $j$ and $d v$ is the corresponding volume form. For any $C^{1}$ map $f$, we have point-wise identity

$$
\left\langle\bar{\partial}_{J} f, K_{J}(f, \alpha)\right\rangle=f^{*} \alpha
$$

Proof. Fix a point $p$ in $\Sigma$, and choose an orthonormal basis $\left\{e_{+}, e_{-}=j e_{+}\right\}$ of $T_{p} \Sigma$. Set $v_{ \pm}=d f\left(e_{ \pm}\right)$, then direct computation gives $\bar{\partial}_{J} f\left(e_{ \pm}\right)=\frac{1}{2}\left(v_{ \pm} \pm\right.$ $\left.J v_{\mp}\right)$, and $K_{J}(f, \alpha)\left(e_{ \pm}\right)=\frac{1}{2}\left( \pm K_{\alpha} v_{\mp}-J K_{\alpha} v_{ \pm}\right)$. The proposition follows from direct computation.

One of the key observation is the following: Assume $f^{\prime}$ is $(J, \alpha)$-holomorphic 
and in the same relative class as $f$, then by proposition 5.13 ,

$$
\begin{aligned}
\int_{\left(\Sigma^{\prime}, \partial \Sigma^{\prime}\right)}\left|\bar{\partial} f^{\prime}\right|^{2} d v & =\int_{\left(\Sigma^{2}, \partial \Sigma^{2}\right)} g\left(\bar{\partial} f^{\prime}, K_{J}\left(f^{\prime}, \alpha\right)\right) d v \\
& =\int_{\left(\Sigma^{\prime}, \partial \Sigma^{\prime}\right)} f^{\prime *} \alpha \\
& =\int_{(\Sigma, \partial \Sigma)} f^{*} \alpha+\int_{C} d F^{*} \alpha+\int_{C^{\prime}} F^{*} \alpha=0
\end{aligned}
$$

where $F$ is the homotopy between $f$ and $f^{\prime}$. The first term vanishes because $f$ is $J$-holomorphic while the second term vanishes because $\Omega$ is $d$-closed, the last term is from boundary of domain and vanishes because $\left.\alpha\right|_{L}=0$. Therefore, $\bar{\partial} f^{\prime}=0$.

Proposition 5.14. Given any relative class $\gamma \in H_{2}(X, L)$, there is at most one complex structure in the twistor $\mathbb{P}^{1}$ such that $\gamma$ has a holomorphic representative. In particular, any holomorphic Riemann surface with boundary on the special Lagrangian in a K3 surface is rigid in the $S^{1}$-family.

Proof. It is easy to see that $D \bar{\partial}_{\text {new }}$ is a Fredholm operator. Assume $f$ is both $J$ and $(J, \alpha)$-holomorphic and $[\operatorname{Im}(f)]=\gamma$, then $K_{J}(\partial f \circ j)=0$ or $\operatorname{Im}(\partial f) \subseteq \operatorname{Ker}\left(K_{\alpha}\right)$. If $[f] \neq 0$, then there exists holomorphic $v$ such that $d f(v) \neq 0$. For each nonzero $\alpha \in \mathbb{R} \operatorname{Im} \Omega_{\vartheta}, \alpha$ is a symplectic 2 -form (here we use the fact $X$ is a hyperKähler). Therefore, $\alpha(u, d f(v)) \equiv 0$ implies $\alpha=0$. The only possible complex structure is $-J$ but it will only realize $-[\gamma]$ as holomorphic cycle.

Therefore, the moduli space for family $\mathcal{M}_{k+1, \beta}(\mathfrak{X}, L)$ has the same underlying space as the usual moduli space of holomorphic discs $\mathcal{M}_{k+1, \beta}\left(X_{\vartheta}, L\right)$ 
for some $\vartheta \in S^{1}$. However, we will equip it with different Kuranishi structure in the next section.

Proposition 5.15. $\operatorname{Im} D \bar{\partial} \cap\left\{K_{J}(f, \alpha) \mid \alpha \in \mathbb{R} \operatorname{Im} \Omega^{\prime}\right\}=\{0\}$. In particular, There is a non-trivial map $T S^{1} \rightarrow L^{p}\left(f^{*} T X \otimes \Lambda^{0,1}\right)$ induced from the $S^{1}$ family of hyperKähler manifolds.

Proof. Assuming $f$ is $J$-holomoprhic, then from the Proposition 5.14 one has

$$
\int_{\Sigma} g\left(D \bar{\partial} V, K_{J}(f, \alpha)\right) d v=-\int_{\Sigma} g\left(\bar{\partial} f, K_{J}(V, \alpha)\right) d v+\int_{\Sigma} V^{*} \alpha=0+0=0
$$

for any $V \in \mathcal{A}\left(f^{*} T X,(\partial f)^{*} T L\right)$. The first term is well-defined by Holder's inequality and last term is zero because $\left.\alpha\right|_{L}=0$.

Remark 5.16. All the argument in this section also apply to any symplectic almost Calabi-Yau 2-fold $X$, i.e. $X$ is a symplectic 4-manifold with an almost complex structure $J$ such that there exists a $J$-holomorphic 2-form $\Omega$ and $J$ induces a Riemannian metric.

\subsection{Kuranishi Structure for Moduli Space of Holomorphic Discs in $S^{1}$-Family}

The main goal of this section is to develop the "reduced" Kuranishi structure,which help to define the new invariant, from the $S^{1}$-family of complex structure. The construction is a modified from [7] and [12].

Theorem 5.17. There exists a Kuranishi structure admits required properties listed below: 
1. It is compatible with the forgetful maps for each $k \geq 1$,

$$
\mathfrak{f o r g e t}_{k, 0}: \mathcal{M}_{k, \beta}(\mathfrak{X}, L) \rightarrow \mathcal{M}_{k-1, \beta}(\mathfrak{X}, L)
$$

2. For each $k \geq 1$, the evaluation map $\left\{e v_{i}, e v_{\vartheta}\right\}: \mathcal{M}_{k, \beta}(\mathfrak{X}, L) \rightarrow L^{k} \times S_{\vartheta}^{1}$ are weakly submersive. Thus the fibre product of Kuranishi structure in 4. make sense.

3. It is invariant under the cyclic permutation of the boundary marked points.

4. For the decomposition of the boundary of moduli spaces, the restriction of the Kuranishi structure on the boundary hand side coincides with the fibre product of the Kuranishi structures of the decomposition.

Proof. Step 1: For each point $\mathbf{p}=f:\left(\Sigma=\cup \Sigma_{a}, \partial \Sigma\right) \rightarrow(X, L)$ (we include $\vec{z}$ in $\Sigma$ for simplicity) in the moduli space $\mathcal{M}_{k, \beta}(\mathfrak{X}, L)$, we will construct a Kuranishi neighborhood. We first consider the case when the domain is stable, namely, then the automorphism of each component is finite. Let

$$
W^{1, p}\left(f^{*} T X,(\partial f)^{*} T L\right)=\left\{\left(v_{a}\right) \in \oplus_{a} W^{1, p}\left(\Sigma_{a} ; f^{*} T X,(\partial f)^{*} T L\right) \mid\right.
$$

$v_{a}$ coincides on nodes $\}$,

where $v_{a} \in W^{1, p}\left(\Sigma_{a} ; f^{*} T X,(\partial f)^{*} T L\right)$ satisfies $v_{a} \in W^{1, p}\left(\Sigma_{a}, f^{*} T X\right)$ and $\left.v_{a}\right|_{\partial \Sigma_{a}} \in W^{1-1 / p, p}\left(\partial \Sigma_{a}, f^{*} T L\right)$. We may choose $p$ large enough such that $v_{a}$ is continuous on $\Sigma_{a}$. 
We consider the following linearized operator

$$
\begin{aligned}
& D \bar{\partial}_{n e w}: W^{1, p}\left(f^{*} T X,(\partial f)^{*} T L\right) \times \mathbb{R}_{\vartheta} \rightarrow L^{p}\left(f^{*} T X \otimes \Lambda^{0,1}\right) \\
&(w, \vartheta) \mapsto D \bar{\partial} w+\vartheta K_{J}(f, \alpha)
\end{aligned}
$$

Notice that we use $D \bar{\partial}_{\text {new }}$ instead of $\left\{D \bar{\partial}_{\vartheta}\right\}$. It make sense because from from Proposition A.8, we know that $D \bar{\partial}_{n e w}(V, \vartheta)=0$ if and only if $D \bar{\partial} V=0$ together with $\vartheta=0$. Since $D \bar{\partial}_{\text {new }}$ is also Fredholm, one can choose $E_{\mathbf{p}}$ such that

1. $E_{\mathbf{p}}$ is a finite dimensional (complex) subspace of $L^{p}\left(\Sigma, w^{*} T X \otimes \Lambda^{0,1}\right)$.

2. $\operatorname{Im} D \bar{\partial}_{n e w}+E_{\mathbf{p}}=L^{p}\left(\Sigma, w^{*} T X \otimes \Lambda^{0,1}\right)$.

3. Elements of $E_{\mathbf{p}}$ has support away from special points on $\partial \Sigma$.

4. $E_{\mathbf{p}}$ is preserved under $\Gamma_{\mathbf{p}}=\operatorname{Aut}(\mathbf{p})$.

We may enlarge the obstruction bundle $E$ such that $\left(e v_{0}, e v_{\vartheta}\right)$ is weakly submersive. Given any small smooth deformation $f^{\prime}$ of $f$, we can find a diffeomorphism of the domain

$$
I_{f, f^{\prime}}: \Sigma \rightarrow \Sigma^{\prime}
$$

such that $I_{f, f^{\prime}}$ smoothly depends on the domain of $f^{\prime}$ and $I_{f, f^{\prime}}$ is identity on a compact set away from marked points.

We choose a unitary connection on TX such that L is totally geodesic 
and thus we can have a identification of obstruction bundle

$$
L^{0, p}\left(\Sigma, f^{*}\left(T X \otimes \Lambda^{0,1}\right) \cong L^{0, p}\left(\Sigma^{\prime}, f^{\prime *}\left(T X \otimes \Lambda^{0,1}\right)\right.\right.
$$

induced by $I_{f, f^{\prime}}$ and again denoted by the same notation. Set $E_{f^{\prime}}=$ $I_{f, f^{\prime}}\left(E_{\mathbf{p}}\right)$ and we consider the equation

$$
\bar{\partial} f^{\prime} \equiv 0 \bmod E_{f^{\prime}}
$$

Let $U_{f}$ be the solutions of (31) and it is a smooth manifold of dimension $\operatorname{dim} E_{\mathbf{p}}$ by implicit function theorem. We define the section of Kuranishi chart by

$$
s\left(f^{\prime}\right)=\bar{\partial} f^{\prime} \in E_{f^{\prime}}
$$

Notice that $\mathcal{M}_{\beta}(\mathfrak{X}, L)=\mathcal{M}_{\beta}\left(X_{\vartheta}, L\right)$ for some $\vartheta \in S^{1}$ as a topological space, therefore the one the same gluing analysis as the standard one. It greatly reduce the complexity of constructing Kuranishi structure. By using Taube's gluing construction and Newton's method we have

Proposition 5.18. Assume $\Sigma^{\prime}$ is a deformation of $\Sigma, f^{\prime}: \Sigma^{\prime} \rightarrow(X, L)$ is the approximate solution and

$$
(w, \vartheta) \in \operatorname{Ker}\left(\pi_{\Sigma^{\prime}} \circ D \bar{\partial}_{n e w}\right), \quad\|(w, \vartheta)\|_{W^{1, p}} \leq \epsilon
$$


then there exists a unique $h_{\left(\Sigma^{\prime}, V\right)} \in L^{p}\left(\Sigma^{\prime} ; w^{\prime *} T X \otimes \Lambda_{\Sigma^{\prime}}^{0,1}\right)$ such that

$$
\pi_{\Sigma^{\prime}} \circ\left(\bar{\partial} \exp _{w^{\prime}}\left(w+Q_{\Sigma^{\prime}} h_{(\Sigma, V)}\right)+K_{J}\left(\left(\exp _{w^{\prime}}\left(w+Q_{\Sigma^{\prime}} h_{(\Sigma, V)}\right), \alpha\right)\right)\right)=0
$$

and

$$
\left\|h_{\left(\Sigma^{\prime}, V\right)}\right\|_{L^{p}} \leq \epsilon^{\prime}
$$

Proof. This is a modified version of Proposition 6.32 [28]. We will use Newton's method to find exact solution. For simplicity of notations, we write $v$ for $f^{\prime}, Q$ for the right inverse for $f^{\prime}, D_{n}$ for linearization of $\bar{\partial}$ on $v_{n}=\exp _{v}\left(w+Q h_{n}\right)$ and $\pi_{n}=\pi_{v_{n}}$. Set $h_{0}=0$ and

$$
h_{n+1}=h_{n}-P_{n} \circ \pi\left(\bar{\partial} v_{n}+\vartheta K_{J}\left(v_{n}, \alpha\right)\right) \text {, }
$$

where $P_{n}$ is the parallel transport along the geodesic $t \in[0,1] \mapsto \exp _{v}((1-$ t) $\left.w+Q h_{n}\right)$.

$$
\begin{aligned}
& P_{n+1} \circ \pi \circ\left(\bar{\partial} v_{n+1}+\vartheta K_{J}\left(v_{n+1}, \alpha\right)\right) \\
& =P_{n+1} \circ \pi \circ\left(\bar{\partial} \exp _{v}\left(w+Q h_{n}-Q \circ P_{n} \circ \pi\left(\bar{\partial} v_{n}+\vartheta K_{J}\left(v_{n}, \alpha\right)\right)\right)\right. \\
& \left.\quad+\vartheta K_{J}\left(\exp _{v}\left(w+Q h_{n}-Q \circ P_{n} \circ \pi\left(\bar{\partial} v_{n}+\vartheta K_{J}\left(v_{n}, \alpha\right)\right)\right), \alpha\right)\right) \\
& =P_{n} \circ \pi \circ\left(\bar{\partial} v_{n}+\vartheta K_{J}\left(v_{n}, \alpha\right)\right) \\
& -P_{n} \circ \pi_{n} \circ D_{n} \circ\left(d \exp _{v}\right)_{\left(w+Q h_{n}\right)}\left(Q \circ P_{n} \circ \pi\left(\bar{\partial} v_{n}+\vartheta K_{J}\left(v_{n}, \alpha\right)\right)\right) \\
& +P_{n} \circ \pi_{n} \circ \vartheta K_{J}\left(D_{n} \circ\left(d \exp _{v}\right)_{\left(w+Q h_{n}\right)}\left(Q \circ P_{n} \circ \pi\left(\bar{\partial} v_{n}+\vartheta K_{J}\left(v_{n}, \alpha\right)\right)\right), \alpha\right) \\
& +R\left(\pi \circ\left(\bar{\partial} v_{n}+\vartheta K_{J}\left(v_{n}, \alpha\right)\right)\right),
\end{aligned}
$$


where

$\left\|R\left(\pi \circ\left(\bar{\partial} v_{n}+\vartheta K_{J}\left(v_{n}, \alpha\right)\right)\right)\right\|_{L^{p}} \leq C_{1}\left\|\pi \circ\left(\bar{\partial} v_{n}+\vartheta K_{J}\left(v_{n}, \alpha\right)\right)\right\|_{L^{p}}^{2}$,

$\left\|P_{n} \circ \pi_{n} \circ D_{n} \circ\left(\operatorname{dexp}_{v}\right)_{\left(w+Q h_{n}\right)}-\pi \circ D_{v}\right\| \leq C_{2}\left(|w|+\left|Q h_{n}\right|\right)$,

(the third term after the second equality) $\leq|\vartheta|\left\|\pi\left(\bar{\partial} v_{n}+\vartheta K_{J}\left(v_{n}, \alpha\right)\right)\right\|_{L^{p}}$.

Therefore, by induction we have

$$
\begin{gathered}
\left\|\pi\left(\bar{\partial} v_{n+1}+K_{J}\left(v_{n+1}, \alpha\right)\right)\right\|_{L^{p}} \\
\leq C_{3}\left(|w|+\left|Q h_{n}\right|+|\vartheta|+\left\|\pi\left(\bar{\partial} v_{n}+K_{J}\left(v_{n}, \alpha\right)\right)\right\|_{L^{p}}\right) \\
\left.\cdot \| \pi\left(\bar{\partial} v_{n}+K_{J}\left(v_{n}, \alpha\right)\right)\right) \|_{L^{p}}
\end{gathered}
$$

From the recursion expression

$$
h_{n}=-\sum_{k=0}^{n-1} P_{k} \circ \pi \circ\left(\bar{\partial} v_{k}+K_{J}\left(v_{k}, \alpha\right)\right) \text {, }
$$

we have control on

$$
\begin{aligned}
& \left\|\pi \circ\left(\bar{\partial} v_{n+1}+\vartheta K_{J}\left(v_{n+1}, \alpha\right)\right)\right\|_{L^{p}} \\
\leq & C_{3}\left(\|(w, \vartheta)\|_{W^{1, p}}+\sum_{k=0}^{n}\left\|\pi\left(\bar{\partial} v_{k}+\vartheta K_{J}\left(v_{k}, \alpha\right)\right)\right\|\right)\left\|\bar{\partial} v_{n}+\vartheta K_{J}\left(v_{n}, \alpha\right)\right\| .
\end{aligned}
$$

It is easy to prove following lemma by induction. 
Lemma 5.19. Assume $a_{n} \geq 0, b>0$ with $a_{0}, b>\frac{1}{6 C_{3}}$. If

$$
a_{n+1} \leq C_{3}\left(b+\sum_{k=0}^{n} a_{k}\right) a_{n} \Longrightarrow a_{n+1} \leq \frac{1}{2} a_{n}
$$

Lemma 5.20. (Lemma 7.1.29 [12]) Assume that $\pi\left(\bar{\partial} w+\vartheta K_{J}(u, \alpha)\right)=0$, then

$$
\left\|\pi\left(\bar{\partial} v+\vartheta K_{J}(v, \alpha)\right)\right\|_{L^{p}} \leq C_{5}\left(\left(|\eta|+\left|\eta^{\prime}\right|\right)^{\frac{1}{2 p}}+|\vartheta|\right) .
$$

Therefore, $\left\|\pi\left(\bar{\partial} \exp _{v} w+\vartheta K_{J}(v, \alpha)\right)\right\|_{L^{p}} \leq C_{5}\left(|\eta|+\left|\eta^{\prime}\right|\right)^{\frac{1}{2 p}}+C_{6}\|w\|_{W^{1, p}}$. and small enough choice of $\eta, \eta^{\prime}$ and $(w, \vartheta)$ guarantee the existence of $h_{\eta, \eta^{\prime} ; u}$ by Newton's method with required estimate. Since $\bar{\partial}_{\vartheta} \exp _{v}\left(w+Q h_{n}\right) \in E_{v}$ is smooth, the exact solution $\exp _{v}\left(w+Q h_{n}\right)$ is smooth from elliptic regularity of Cauchy-Riemann operator.

As for uniqueness, let $h=h_{\eta, \eta^{\prime} ; f}$ and assume there another solution $h^{\prime}$ of equation

$$
\pi\left(\bar{\partial} \exp _{v}(w+Q h)+\vartheta K_{J}(v, \alpha)\right)=0, \text { with }\|h\|_{L^{p}} \leq \epsilon
$$


Then we have

$$
\begin{aligned}
0 & =P^{\prime} \circ \pi\left(\bar{\partial} \exp _{v}\left(w+Q h^{\prime}\right)+\vartheta K_{J}\left(\exp _{v}\left(w+Q h^{\prime}\right), \alpha\right)\right) \\
& =P^{\prime} \circ \pi\left(\bar{\partial} \exp _{v}\left(w+Q h+Q\left(h^{\prime}-h\right)\right)+\vartheta K_{J}\left(\exp _{v}\left(w+Q\left(h^{\prime}-h\right)\right)\right)\right) \\
& =P \circ \pi \circ \bar{\partial} \exp _{v}(w+Q h)+P \circ \pi \circ D_{1} \circ\left(\operatorname{dexp}_{v}\right)(w+Q h)\left(Q\left(h^{\prime}-h\right)\right) \\
& +R\left(h^{\prime}-h\right)
\end{aligned}
$$

or

$$
h^{\prime}-h=\left(P_{1} \circ \pi \circ D_{1} \circ\left(\exp _{v}\right)_{(w+Q h)}-\pi \circ D\right)\left(Q\left(h^{\prime}-h\right)\right)+R\left(h^{\prime}-h\right)
$$

Therefore,

$$
\left\|h^{\prime}-h\right\|_{L^{p}} \leq \frac{1}{2}\left\|h^{\prime}-h\right\|_{L^{p}}
$$

when $\epsilon$ is small and $h=h^{\prime}$.

Therefore we construct a Kuranishi chart for each element $\mathbf{p}=[f$ : $((\Sigma, \partial \Sigma), \vec{z}) \rightarrow(X, L)] \in \mathcal{M}_{k, \beta}(\mathfrak{X}, L)$ when the domain is stable.

Step 2: If any of the component of the domain $\Sigma_{a}$ is unstable, we will follow the construction in [7] and Appendix [11]. We first add interior marked points to make $\Sigma_{a}$ stable and denote it by $\Sigma_{a}^{+}$. we add the marked points in the way that $\Gamma_{\mathbf{p}}$ acts on additional marked points freely. Since $f_{a}$ is non-degenerate, it is immersed at generic point on $\Sigma_{a}$ and without loss of generality we can assume $f_{a}$ is immersed at additional marked points. 
For each additional marked point $p$, we take a 2-dimensional submanifold $\mathcal{D}_{p} \subseteq X$ such that $\mathcal{D}_{p}$ intersect with the image of $f_{a}$ transversally at $p$ and $\mathcal{D}_{p}=\mathcal{D}_{\gamma \cdot p}$, for each $\gamma \in \Gamma_{\mathbf{p}}$. We will denote the holomorphic maps with addtion marked points by $\mathbf{p}^{+}=\left[f^{+}:\left(\Sigma^{+}, \partial \Sigma^{+}\right) \rightarrow(X, L)\right]$

Now we may assume the domain with addition marked points $\Sigma^{+} \epsilon$ $\mathcal{M}_{0, k+k^{\prime}}$, where $k^{\prime}$ is the number of the additional marked points. Since $\mathcal{M}_{0, k+k^{\prime}}$ admits an orbifold structure, we may assume a neighborhood of $\Sigma^{+} \in \mathcal{M}_{0, k+k^{\prime}}$ is parametrized by $V\left(\Sigma^{+}\right) / \operatorname{Aut}\left(\Sigma^{+}\right)$. Follow the same procedure in Step 1, we construct an Kuranishi chart $V_{\mathbf{p}^{+}}$for $\mathbf{p}^{+}$. Let $e v_{\text {add }}$ : $V_{\mathbf{p}^{+}} \rightarrow X^{k^{\prime}}$ be the evaluation map of added points. Then

$$
V_{\mathbf{p}}:=V_{\mathbf{p}^{+} e v_{\text {add }} \times} \prod_{\begin{array}{c}
p: \text { additional } \\
\text { marked points }
\end{array}} \mathcal{D}_{p}
$$

is a smooth manifold of expected dimension because of the transversality condition of $\mathcal{D}_{p}$. We define $E_{\mathbf{p}}$ by

$$
E_{f^{\prime}}=E_{\mathbf{p}^{+}}=\bigoplus_{a} E\left(\Sigma_{a}^{+}\right)
$$

(up to a parallel transport) and the Kuranishi map $s_{\mathbf{p}}$ by

$$
s_{\mathbf{p}}\left(f^{\prime}\right)=\bar{\partial} f^{\prime} \in E_{f^{\prime}},
$$

which is $\Gamma_{\mathbf{p}}$-equivariant by construction.

Let $s_{\mathbf{p}}\left(f^{\prime}\right)=0$, then $f^{\prime}:\left(\Sigma_{f^{\prime}}^{+}, \partial \Sigma_{f^{\prime}}^{+}\right) \rightarrow(X, L)$ is pseudo-holomorphic and we get $\psi_{\mathbf{p}}\left(f^{\prime}\right):=\tilde{f}:\left(\Sigma_{f^{\prime}}^{+}, \partial \Sigma_{f^{\prime}}^{+}\right) \rightarrow(X, L)$, where the later is induced 
by $f^{\prime}$ by forgetting those additional marked points. So far, we construct a Kuranishi chart with notation changed by $\left(V_{\mathbf{p}}^{0}, E_{\mathbf{p}}^{0}, \Gamma_{\mathbf{p}}, s_{\mathbf{p}}^{0}, \psi_{\mathbf{p}}^{0}\right)$ for every point $\mathbf{p} \in \mathcal{M}_{k, \beta}(\mathfrak{X}, L)$.

Step 3: By Gromov compactness theorem, we find a finite cover

$$
\mathcal{M}_{k, \beta}(\mathfrak{X}, L)=\bigcup_{\mathbf{p} \in \mathfrak{U}} \psi_{\mathbf{p}}^{0}\left(\left(s_{\mathbf{p}}^{0}\right)^{-1}(0) / \Gamma_{\mathbf{p}}\right)
$$

Choose closed subset $W_{\mathbf{p}}$ of $\psi_{\mathbf{p}}^{0}\left(\left(s_{\mathbf{p}}^{0}\right)^{-1}(0) / \Gamma_{\mathbf{p}}\right)$ for each $\mathbf{p} \in \mathfrak{U}$ such that

$$
\mathcal{M}_{k, \beta}(\mathfrak{X}, L)=\bigcup_{\mathbf{p} \in \mathfrak{U}} \operatorname{Int} W_{\mathbf{p}}
$$

and we set

$$
E \mathbf{p}=\bigoplus_{\mathbf{p}^{\prime} \in \mathfrak{U}(\mathbf{p})} E_{\mathbf{p}^{\prime}}^{0}, \text { where } \mathfrak{U}(\mathbf{p})=\left\{\mathbf{p}^{\prime} \in \mathfrak{U} \mid \mathbf{p} \in W_{\mathbf{p}^{\prime}}\right\}
$$

This step the closeness of $W_{\mathbf{p}}$ guarantee the coordinate change of Kuranishi structure. Thus we construct a Kuranishi structrue on a fixed moduli space $\mathcal{M}_{k, \beta}(\mathfrak{X}, L)$ but without compatibility condition.

Step 4: We will construct the Kuranishi structures on $\mathcal{M}_{k, \beta}(\mathfrak{X}, L)$ inductively on $\omega(\beta)$ such that they are compatible with the decomposition of boundary.

Notice that for the case $[\partial \beta]=0 \in H_{1}(L)$, there is an additional boundary component of $\mathcal{M}_{0, \gamma}(\mathfrak{X}, L)$

$$
\mathcal{M}_{1, \tilde{\beta}}^{c l}(\mathfrak{X}) \times_{M} L
$$


The proof is also similar.

Remark 5.21. It is pointed out in [8] that we choose $E_{\alpha}$ such that its elements has support (uniformly) away from the special points for two reasons:

1. We don't have to perturb in a neighborhood of nodal points and thus the gluing analysis is easier.

2. It is easier to identify the sections of obstruction bundle of each component with the sections of obstruction bundle after gluing.

Remark 5.22. Here we didn't quotient the line bundle given by $K_{J}(f, \alpha)$ because it is hard to identify the quotients with those of perturbation of $f$.

Theorem 5.23. For each $\epsilon$ and $E_{0}$, there exists a system of continuous family of multi-sections $\left\{\mathfrak{s}_{k, \beta}\right\}$ on $\left\{\mathcal{M}_{k, \beta}|k \geq 0,| \int_{\beta} \Omega \mid<\epsilon\right\}$ such that

1. It is $\epsilon$-close to the Kuranishi map in $C^{0}$ sense.

2. It is compatible with forgetful map forget.

3. It is invariant under the cyclic permutation of the boundary marked points.

4. All the relevant evaluation maps induce submersion on zero sets of multi-sections. 
5. For the decomposition of the boundary of moduli spaces,

$$
\begin{array}{r}
\partial \mathcal{M}_{k+1, \beta}(\mathfrak{X}, L)=\bigcup_{1 \leq i \leq j+1 \leq k+1} \bigcup_{\substack{\beta_{1}+\beta_{2}=\beta, Z_{\beta_{1}} / Z_{\beta_{2}} \in \mathbb{R}_{>0}}} \mathcal{M}_{j-1+1, \beta_{1}}(\mathfrak{X}, L) \\
\left(e v_{0}, e v_{\vartheta}\right) \\
\times_{\left(e v_{i}, e v_{\vartheta}\right)} \\
\mathcal{M}_{k-j+1, \beta_{2}}(\mathfrak{X}, L) .
\end{array}
$$

and

$$
\begin{aligned}
& \partial \mathcal{M}_{0, \beta}(\mathfrak{X}, L)=\bigcup_{\tilde{\beta}: i_{*}(\tilde{\beta})=\beta}\left(\mathcal{M}_{1, \tilde{\beta}}^{c l} \underset{\mathfrak{X}}{\times}\left(L \times S_{\vartheta}^{1}\right)\right) \\
& \cup \bigcup_{\substack{\beta_{1}+\beta_{2}=\beta, Z_{\beta_{1}} / Z_{\beta_{2}} \in \mathbb{R}_{>0}}}\left(\mathcal{M}_{1, \beta_{1}}(\mathfrak{X}, L)_{\left(e v_{0}, e v_{\vartheta}\right)} \times_{\left(e v_{0}, e v_{\vartheta}\right)} \mathcal{M}_{1, \beta_{2}}(\mathfrak{X}, L)\right) / \mathbb{Z}_{2}
\end{aligned}
$$

the restriction of the multi-sections on the boundary of the moduli space coincide with the fibre product of the multi-sections from decomposition.

Proof. The proof is similar to theorem 5.1 [7]. By Gromov compactness, the class achieve minimal area has compact moduli space consisting only smooth domain. One constructs multi-sections on the moduli space using appendix A.3. By induction, we have constructed multi-sections on both factor of right hand side of (33) for $\beta$ with $\left|Z_{\beta}\right|<E_{0}$. For second terms on the right hand side of (33) and right hand side of (5), multi-sections constructed are compatible on the overlapped part of moduli spaces by lemma 7.2.55 [12]. Therefore, we can use the fibre product of multi-sections on each factor to gives multi-sections on the left hand side of (12),(33). For the first term 
of right hand side, [11] constructs such multi-sections. We use lemma 3.14 [11] to extend the multi-sections from the boundary to the whole moduli space and the multi-sections can satisfy (45). It is easy to see that the extension still has the transversal property. The assumption of energy bound guarantees we only have finitely many steps so that the (1) of Theorem 5.23 can be achieved.

We will use $[\mathcal{M}]^{v i r}$ to denote the zero locus of the perturbed multisections of corresponding moduli space $\mathcal{M}$. In particular, $[\mathcal{M}]^{\text {vir }}$ is a smooth manifold (with corners).

Remark 5.24. See 7.2.3 [12] explains why we can not construct multisection for all moduli space simultaneously.

\subsection{Orientation}

Since the tangent bundle of torus is trivial, one can choose the trivial spin structure and is invariant under the monodromy. Let $u:\left(D^{2}, \partial D^{2}\right) \rightarrow$ $(X, L)$ be a holomorphic map with image in the relative class $\gamma$. Assume the domain of the disc is given by $D^{2}=\{z \in \mathbb{C}:|z| \leq 1\}$. We shrink the circle $|z|=1-\epsilon$, where $\epsilon$ is a small positive number. Let $C$ be the resulting rational curve and intersect a disc $D^{\prime 2}$ at a point $p$. The bundle pair $\left(u^{*} T X, u^{*} T L\right)$ is trivial on $D^{\prime 2}$ and has natural orientation. Following Chapter 8 [12], we can define the orientation of standard moduli space $\mathcal{M}_{0, \gamma}(X, L)$ by the orientation of virtual vector space

$$
H^{0}\left(\Sigma, \partial \Sigma ; u^{*} T X, u^{*} T L\right)-H^{1}\left(\Sigma, \partial \Sigma ; u^{*} T X, u^{*} T L\right)
$$


From the below long exact sequence we have a canonical choice of orientation on the mouldi space since every term below admits a natural orientation.

$$
\begin{aligned}
0 & \rightarrow H^{0}\left(\Sigma, \partial \Sigma ; u^{*} T X, u^{*} T L\right) \rightarrow H^{0}(C, F) \oplus H^{0}\left(D^{2}, \partial D^{2} ; \mathbb{C}^{2}, \mathbb{R}^{2}\right) \rightarrow \mathbb{C}^{2} \\
& \rightarrow H^{1}\left(\Sigma, \partial \Sigma ; u^{*} T X, u^{*} T L\right) \rightarrow H^{1}(C, F) \oplus H^{1}\left(D^{2}, \partial D^{2} ; \mathbb{C}^{2}, \mathbb{R}^{2}\right) \rightarrow 0
\end{aligned}
$$

where $F$ is a degree zero rank 2 bundle over $C$ induced from $\left(u^{*} T X, u^{*} T L\right)$. For our moduli space $\mathcal{M}_{0, \gamma}(\mathfrak{X}, L)$, we define its orientation to be

$$
S_{\vartheta}^{1} \times\left(H^{0}\left(\Sigma, \partial \Sigma ; u^{*} T X, u^{*} T L\right)-H^{1}\left(\Sigma, \partial \Sigma ; u^{*} T X, u^{*} T L\right)\right) / \operatorname{Aut}\left(D^{2}\right) .
$$

Remark 5.25. Each initial ray corresponding to the singularity has same orientation.

\subsection{Reduced $A_{\infty}$ Structure and Floer Theoretic Counting}

From the Kuranishi strucuture constructed in the previous section, one can define a filtered $A_{\infty}$ structure using De Rham model as follows: Let $\rho_{k} \in$ $\Lambda^{*}\left(L \times S_{\vartheta}^{1}\right)$ be differential forms on $L \times S_{\vartheta}^{1}$. For each $k \geq 1$, we define

$$
m_{k, \beta}\left(\rho_{1}, \cdots, \rho_{k}\right) \in \Lambda^{*}\left(L \times S_{\vartheta}^{1}\right), \quad *=\sum_{i=1}^{k}\left(\operatorname{deg}\left(\rho_{i}\right)-1\right)+1
$$




$$
\begin{gathered}
m_{k, \beta}\left(\rho_{1}, \cdots, \rho_{k}\right)=\operatorname{Corr}_{*}\left(\mathcal{M}_{k+1, \beta}(\mathfrak{X}, L) ;\left(e v_{1}, \ldots, e v_{k}, e v_{\vartheta}\right),\left(e v_{0}, e v_{\vartheta}\right)\right) \\
\left(\rho_{1} \times \cdots \times \rho_{k}\right), \\
m_{0, \beta}(1)=\operatorname{Corr}_{*}\left(\mathcal{M}_{1, \beta}(\mathfrak{X}, L) ; \operatorname{tri},\left(e v_{0}, e v_{\vartheta}\right)\right)(1) \in \Lambda^{2}\left(L \times S_{\vartheta}^{1}\right) \\
\mathfrak{m}_{k}=\sum_{\beta \in \pi_{2}(X, L)} m_{k, \beta} T^{\int_{\gamma} \Omega}
\end{gathered}
$$

and if $X$ is an elliptic K3 surface and $L$ is the torus fibre we define

$$
m_{-1, \beta}=\operatorname{Corr}_{*}\left(\mathcal{M}_{0, \beta}(\mathfrak{X}, L) ; \text { tri,tri }\right)(1) \in \mathbb{R}
$$

Theorem 5.26. $\left\{m_{k, \beta}\right\}_{k \geq 0}$ forms a cyclic filtered $A_{\infty}$ algebra structure modulo $T^{E_{0}}$ on $\Lambda\left(L \times S_{\vartheta}^{1}\right)$ with 1 as a strict unit. Moreover, the structure is independent of the choice of Kuranishi structure and multi-sections chosen, up to pseudo-isotopy of inhomogeneous cyclic filtered $A_{\infty}$ algebras.

Proof. This is a standard argument follow [12]. However, the new Kuranishi structure induce a new $A_{\infty}$ structure and can be viewed as a new symplectic invariant. We first will construct an inhomogeneous cyclic filtered $A_{\infty}$ algebra structure modulo $T^{E_{0}}$. From the Kuranishi structure and multi-section constructed in Theorem 5.23, we first prove the $A_{\infty}$ relation modulo $T^{E}$. The $A_{\infty}$ relation is equivalent to

$$
\begin{gathered}
\sum_{\beta_{1}+\beta_{2}=\beta} \sum_{k_{1}+k_{2}=k+1} \sum_{i}(-1)^{\operatorname{deg}\left(\rho_{1}\right)+\cdots+\operatorname{deg}\left(\rho_{i-1}\right)+i-1} \\
m_{k_{1}, \beta_{1}}\left(\rho_{1}, \cdots, m_{k_{2}, \beta_{2}}\left(\rho_{i}, \cdots, \rho_{i+k_{2}-1}\right), \cdots, \rho_{k}\right)=0
\end{gathered}
$$


We may write the sum from left hand side into

$$
\begin{array}{r}
m_{1,0} m_{k, \beta}\left(\rho_{1}, \cdots, \rho_{k}\right) \\
+\sum_{i}(-1)^{\operatorname{deg}\left(\rho_{1}\right)+\cdots+\operatorname{deg}\left(\rho_{i-1}\right)+i-1} m_{k, \beta}\left(\rho_{1}, \cdots, m_{1,0}\left(\rho_{i}\right), \cdots, \rho_{k}\right) \\
+\sum_{\substack{\beta_{1}+\beta_{2}=\beta, k_{1}+k_{2}=k+1 \\
\beta_{1} \neq 0 \text { or } k_{1} \neq 1 \\
\beta_{2} \neq 0 \text { or } k_{2} \neq 1}} \sum_{i}(-1)^{\operatorname{deg}\left(\rho_{1}\right)+\cdots+\operatorname{deg}\left(\rho_{i-1}\right)+i-1} \\
m_{k_{1}, \beta_{1}}\left(\rho_{1}, \cdots, m_{k_{2}, \beta_{2}}\left(\rho_{i}, \cdots, \rho_{i+k_{2}-1}\right), \cdots, \rho_{k}\right)
\end{array}
$$

We need the version of Stoke's theorem A.14 and composition law A.15 for manifolds with Kuranishi structure.

We need to check the orientation of the moduli space to fix the sign. For $0<E_{0}<E_{1}$, one can extend the $A_{\infty}$ relation from modulo $T^{E_{0}}$ to modulo $T^{E_{1}}$.

The cyclic symmetry follows from the cyclic symmetry of the perturbed multi-sections:

$$
\begin{aligned}
& \left\langle m_{k+1, \beta}\left(\rho_{1}, \cdots, \rho_{k}\right), \rho_{0}\right\rangle \\
& \quad=\operatorname{Corr}_{*}\left(\mathcal{M}_{k+1, \beta}(\mathfrak{X}, L) ;\left(e v_{1}, \cdots, e v_{k}, e v_{0}\right), \operatorname{tri}\right)\left(\rho_{1} \times \cdots \rho_{k} \times \rho_{0}\right)
\end{aligned}
$$

To prove 1 is a strict unit: for $\beta \neq 0$ and $k \leq 1$, we want to claim

$$
m_{k, \beta}\left(\rho_{1}, \cdots, \rho_{i-1}, 1, \rho_{i+1}, \cdots, \rho_{k}\right)=0
$$

This is because of the compatibility of forgetful map forget $: \mathcal{M}_{k} \rightarrow \mathcal{M}_{k-1}$ 
Let $X$ be the vector tangent to the fibre of the forgetful map forget, then

$$
\iota_{X}\left(\left(f^{s}\right)_{\alpha}^{*} \rho \wedge \omega\right)=0
$$

where $\rho=\rho_{1} \times \cdots \times \rho_{i-1} \times 1 \times \rho_{i+1} \times \cdots \rho_{k}$. Therefore each components of right hand side of 5.5 vanish and 1 is a strict unit.

Remark 5.27. Here we need to include the factor $S_{\vartheta}^{1}$ to make $m_{k}$ degree 1 . Otherwise, $m_{k}$ will be degree 0 and the $A_{\infty}$-structure is destroyed.

Proposition 5.28. The $A_{\infty}$-structure $\left(\Lambda\left(L \times S_{\vartheta}^{1}\right),\left\{m_{k, \beta}\right\}\right)$ constructed in Proposition 5.26 is independent of choice of Kuranishi structure and the family of multi-section chosen, up to pseudo-isotopy of inhomogeneous cyclic filtered $A_{\infty}$ algebra modulo $T^{E_{0}}$

Proof. The proof is similar to proposition $4.1[8]$

Finally we apply lemma $4.2[8]$ to extend the inhomogeneous cyclic filtered $A_{\infty}$ algebra structure and finish the proof of Theorem 5.26.

Remark 5.29. Let $\mathcal{X}$ be the twistor space of $X$ with two fibres admit elliptic fibrations discarded. From the expression (1), $\Omega(\zeta) \wedge \frac{d \zeta}{\zeta}$ is a nowhere vanishing holomorphic $(3,0)$-form on $\mathcal{X}$. Notice that every holomorphic disc $\left(D^{2}, \partial D^{2}\right) \rightarrow\left(\mathcal{X}, L \times S_{\vartheta}^{1}\right)$ factor through a fibre by maximal principle. Theorem 5.26 is equivalent to the Kuranishi structure of the real 3-torus $L \times S_{\vartheta}^{1}$ in $\mathcal{X}$ constructed in [12].

Remark 5.30. One can generalized theorem to the case includes interior marked points and study open-closed duality. 
From now on, we will assume $X$ is an elliptic K3 surface and $L=L_{u}$ is a torus fibre for our purpose. After hyperKähler rotation, $X$ admits an $S_{\vartheta}^{1}$-family of special Lagrangian torus fibration.

Lemma 5.31. Assume $L_{u}$ does not falls on the $W_{\beta}^{\prime}$, then $m_{-1, \beta}$ is welldefined.

Proof. Assume there are two different Kuranishi structures. We can construct a pseudo-homotopy between them. Let $0 \leq t_{1} \leq t_{2} \leq 1$,

$$
\begin{aligned}
& \partial\left(\left[t_{1}, t_{2}\right] \times \mathcal{M}_{0, \beta}\right)=\left(\left\{t_{1}, t_{2}\right\} \times \mathcal{M}_{0, \beta}\right) \cup \underset{\tilde{\beta}: i_{*}(\tilde{\beta})=\beta}{\bigcup_{1}}\left[t_{1}, t_{2}\right] \times\left(\mathcal{M}_{1, \tilde{\beta}}^{c l} \times \mathfrak{X} L \times S_{\vartheta}^{1}\right) \\
& \cup \bigcup_{\beta_{1}+\beta_{2}=\beta}\left(\left(\left[t_{1}, t_{2}\right] \times \mathcal{M}_{1, \beta_{1}}\right)\left(e v_{0}, e v_{\vartheta}, e v_{t}\right) \times\left(e v_{0}, e v_{\vartheta}, e v_{t}\right)\left(\left[t_{1}, t_{2}\right] \times \mathcal{M}_{1, \beta_{2}}\right)\right) / \mathbb{Z}_{2}
\end{aligned}
$$

Note that the middle term has no contribution because of dimension reason. It coincide with the expectation that there are no BPS of pure flavor charges. Apply the Stoke's theorem above, we get

$$
\mathfrak{m}_{-1, \beta}^{1}-\mathfrak{m}_{-1, \beta}^{0}=\text { contribution from right hand side of (37). }
$$

Because of the assumption that $L_{u}$ does not fall on $W_{\beta}^{\prime}$ except multiple cover occur. By Proposition 5.43, the boundary of multiple covers doesn't contribute to the invariant. The finiteness follows from Gromov compactness theorem.

In particular, locally there is an open dense set such $m_{-1, \beta}$ is well-defined. 
By Gromov's compactness theorem (see remark of Theorem 0.2 [39]) and the lemma, the wall of a fixed charge $\beta$ is locally finite. Therefore,

Proposition 5.32. If $m_{-1, \beta}\left(L_{u}\right)$ is well-defined, then $m_{-1, \beta}\left(L_{u^{\prime}}\right)$ is welldefined for nearby $u^{\prime}$.

Finally, we define

$$
\tilde{\Omega}^{\text {Floer }}(\gamma ; u)=m_{-1, \gamma}\left(L_{u}\right) .
$$

A priori, the "invariants" are only defined over $\mathbb{R}$.

Proposition 5.33. If $\tilde{\Omega}^{\text {Floer }}(\gamma ; u)$ is well-defined, then $\tilde{\Omega}^{\text {Floer }}(-\gamma ; u)$ is also well-defined. Moreover, we have reality condition

$$
\tilde{\Omega}^{\text {Floer }}(\gamma ; u)=\tilde{\Omega}^{\text {Floer }}(-\gamma ; u)
$$

Proof. From (34), complex conjugation does not change orientation of $\mathbb{C}^{2}$ ,L, $H^{0}(C, F)-H^{1}(C, F), K_{J}(f, \alpha)$ and $P S L(2, \mathbb{R})$.

Proposition 5.34. The invariants $\Omega(\gamma ; u)$ is invariant if the elliptic fibration changed to its complex conjugate. It is also independent of the choice of the holomorphic $(2,0)$-form of the elliptic fibration.

Proof. If we change the elliptic fibration to its conjugate complex strucuture, then both $S_{\vartheta}^{1}$ and $L$ change orientation. All the other ingredients involve orientation remain the same. This indicates the first part of the proposition. For the later part, we first look at the case when the holomorphic $(2,0)$ form $\Omega$ replaced by $-\Omega$. In this case, the complex structure of the special 
Lagrangian fibration changed to its conjugate. Thus the The sign may change on $H^{0}(C, F)$ and $H^{1}(C, F)$ but cancel out by Riemann-Roch formula. Notice that the orientation of $S_{\vartheta}^{1}$ is unchanged because both $\alpha=\operatorname{Im} \Omega$ and $j$ change sign and $K_{J}(f, \alpha)=\frac{1}{2} K_{\alpha}(d f \circ j)$ is unchanged. In general, any two choices of holomorphic volume form will give rise to pseudo-isotopy of Kuranishi structure and the proof is the same as Lemma 5.31.

Remark 5.35. Considering $S^{1}$-family not only raises the virtual dimension of moduli space to 0 but after projecting to a fixed phase still get the reduced counting.

Let $\pi: X \rightarrow B$ to be the special Lagrangian fibration. Given a wall $W_{\alpha, \beta}$ on the affine base, we choose a path on $B_{0}$ passing through a generic point of $W_{\alpha, \beta}$ and the boundary points $L_{0}=L, L_{1}$ lies on different side of the wall. Without lose of generality, we may Assume the phase $\operatorname{Arg} Z_{\alpha}$ is strictly decreasing and $\operatorname{Arg} Z_{\beta}$ is strictly increasing. Choose a 1-parameter family of fibration preserving diffeomorphisms $\phi_{t}$ such that $\phi_{t}\left(L_{0}\right)=L_{t}$, for $t \in[0,1]$. By pulling back the Kähler forms and complex structures to $L_{0}$, we may view changing special Lagrangian boundary condition as changing complex structures (hyperKähler structures) on $\left(X, L_{0}\right)$. Assume $f:\left(D^{2}, \partial D^{2}\right) \rightarrow$ $(X, L)$ is a holomorphic disc. The new linearized $\bar{\partial}$ equations for 1-parameter family now becomes

$$
\begin{array}{r}
D \bar{\partial}: W^{1, p}\left(f^{*} T X,(\partial f)^{*} T L\right) \times \mathbb{R}_{\vartheta} \times \mathbb{R}_{t} \rightarrow L^{p}\left(f^{*} T X \otimes \Lambda^{0,1}\right) \\
(w, \quad \vartheta, \quad t) \quad \longmapsto D \bar{\partial} w+\vartheta K_{J}(f, \alpha)+t Y
\end{array}
$$


where $Y$ is the tangent of complex moduli along the path. Using similar argument in Theorem 5.23, we have

Theorem 5.36. There is a system of Kuranishi structures and families of multi-sections $\mathfrak{s}_{0, \beta}$ on $\mathcal{M}_{k, \beta}\left(\mathfrak{X},\left\{L_{t}\right\}\right), k=0,1$ for $\left|\int_{\beta} \Omega\right|<E_{0}$ satisfying properties below:

1. The multi-sections $\mathfrak{s}_{k, \beta}$ are transverse to 0 .

2. The structure is compatible with the forgetful maps.

3. All the relevant evaluation maps are submersion restricted on the zero locus of the perturbed multi-sections.

4. For the following decomposition of the boundary of moduli spaces,

$$
\begin{aligned}
\partial \mathcal{M}_{0, \beta}\left(\mathfrak{X},\left\{L_{t}\right\}\right)= & \cup\left(\mathcal{M}_{0, \beta}\left(\mathfrak{X}, L_{1}\right)-\mathcal{M}_{0, \beta}\left(\mathfrak{X}, L_{0}\right)\right) \\
& \cup \bigcup_{t} \bigcup_{\substack{T: \sum_{\beta_{l}=\beta,}, Z_{\beta_{l}}\left(L_{t}\right) / Z_{\beta}\left(L_{t}\right) \in \mathbb{R}_{>0}}} \mathcal{M}_{T}(\mathfrak{X}, L)
\end{aligned}
$$

the restriction of the multi-sections on the boundary of moduli spaces coincides with the fibre products of Kuranishi structures. The summation is over all possible boundary, each of them has a dual intersection complex as a tree $T$. Each such tree $T$ has its vertex $i$ labeled with a charge $\beta_{i} \in H_{2}\left(X, L_{t}\right)$. By Proposition 5.40, we only have to consider those trees with each edge $(i, j)$ has $\left\langle\partial \beta_{i}, \partial \beta_{j}\right\rangle \neq 0$. Let $\mathcal{M}_{T}(\mathfrak{X}, L)$ be the moduli space of holomorphic discs with corresponding configuration. 
5. For $t_{0} \in\{0,1\}$, the induced Kuranishi structures and multi-sections on $\mathcal{M}_{k, \beta}\left(\mathfrak{X},\left\{L_{t}\right\}\right) \cap e v_{t}^{-1}\left(\left\{t_{0}\right\}\right)$ are the one given in Theorem 5.17 and Theorem 5.23.

Remark 5.37. Another choice is to consider the below moduli space in [12].

$$
\mathcal{M}_{k, \beta}\left(\left\{J_{\vartheta, t}\right\}: \operatorname{top}(\vartheta), \operatorname{twp}(t)\right) \rightarrow[0,1]_{t} \times L^{k}
$$

Then the evaluation map $\left(e v_{i}, e v_{\vartheta}\right)$ can be made weakly submersive and one can construct a reduced Kuranishi structures on $\Lambda^{*}\left(L \times S_{\vartheta}^{1}\right)$.

Remark 5.38. Notice that by the Gromov compactness theorem, the last term in (39) is finite.

The following proposition is straight forward from the definition.

Proposition 5.39. Let $\phi$ be an (fibration preserving) diffeomorphism, then $m_{-1, \beta}(L)=m_{-1, \phi(\beta)}(\phi(L))$ assuming both are well-defined.

Proposition 5.40. Assume $\gamma_{1}, \gamma_{2}$ are primitive, then

$$
\operatorname{Corr}_{*}\left(\mathcal{M}_{1, \gamma_{1}}(\mathfrak{X}, L) \times_{L \times S_{\vartheta}^{1}} \mathcal{M}_{1, \gamma_{2}}(\mathfrak{X}, L) ; \text { tri,tri }\right)(1)=\left\langle\gamma_{1}, \gamma_{2}\right\rangle m_{-1, \gamma_{1}} m_{-1, \gamma_{2}}
$$

Proof. It suffices to prove the following statement:

Lemma 5.41. Let $X_{i} \cong B_{i} \times S^{1}$, where $B_{i}$ are manifolds with a compact support volume form $\omega_{i}$. Assume $f_{i}: X_{i} \rightarrow T^{2}$ are submersions such that $\left[f_{1}\left(\{p t\} \times S^{1}\right)\right] \cdot\left[f_{2}\left(\{p t\} \times S^{1}\right)\right]=k$, then $\int_{X} \omega_{1} \wedge \omega_{2}=k\left(\int_{B_{1}} \omega_{1}\right)\left(\int_{B_{2}} \omega_{2}\right)$, where $X=X_{1} \times_{T^{2}} X_{2}$. 
Proof. Consider the map $\pi: X \rightarrow B_{1} \times B_{2}$. $X$ defined by $f_{1}(x)=f_{2}(y)$, is closed and $\pi$ is proper. Easy computation shows that

$$
\int_{X} \omega_{1} \wedge \omega_{2}=(\operatorname{deg} \pi) \cdot \int_{B_{1} \times B_{2}} \omega_{1} \wedge \omega_{2}= \pm k \cdot\left(\int_{B_{1}} \omega_{1}\right)\left(\int_{B_{2}} \omega_{2}\right)
$$

Compatibility of forgetful maps says that if $V_{\alpha_{k}}$ are local Kuranishi chart for $\mathcal{M}_{0, \gamma_{k}}$, for $k=1,2$, then one can take $V_{\alpha_{k}}^{\prime}=V_{\alpha} \times S^{1}$ as Kuranishi chart of $\mathcal{M}_{1, \gamma_{k}}$. Thus locally we can choose $\mathfrak{s}_{\alpha_{k}, i, j}^{-1}(0)$ to be $X_{i}$ in above lemma. $\int_{B_{k}} \omega_{k}$ is the local contribution to $m_{-1, \gamma_{k}}$ and $\int_{X} \omega_{1} \wedge \omega_{2}$ corresponding local contribution for left hand side of (3.13). The proposition followed by using partition of unity for Kuranishi space to glue the contribution from each charts to give the formula.

Remark 5.42. Notice that in the expansion formula of symplectic holomorphic 2-form in (6), there is no contribution from those $\gamma$ with $[\partial \gamma]=0$.

Similar argument as Proposition 5.40, we have follow two propositions.

Proposition 5.43. If $\left\langle\partial \beta_{1}, \partial \beta_{2}\right\rangle_{L}=0$, then

$\operatorname{Corr}_{*}\left(\mathcal{M}_{1, \beta_{1}}\left(\left\{L_{t}\right\}\right)_{\left(e v_{0}, e v_{\vartheta}, e v_{t}\right)} \times_{\left(e v_{0}, e v_{\vartheta}, e v_{t}\right)} \mathcal{M}_{1, \beta_{2}}\left(\left\{L_{t}\right\}\right) / \mathbb{Z}_{2} ;\right.$ tri, tri $)(1)=0$.

Remark 5.44. Proposition 5.43 implies the multiple cover contribution is well-defined and the closed Gromov-Witten invariants decouple from the invariants $\left\{\tilde{\Omega}^{\text {Floer }}\right\}$. Moreover, it implies the analogue of Proposition 3.13 for $\left\{\tilde{\Omega}^{\text {Floer }}\right\}$. 
Proposition 5.45. Let $\left\{L_{t}\right\}$ be a 1-parameter family of torus fibres such that passing through $W_{\gamma}^{\prime \prime}$ transversally. Assume $\gamma=\sum_{i} k_{i} \gamma_{i}$. If

$$
\begin{gathered}
\tilde{\Omega}^{\text {Floer }}\left(k \gamma_{i_{0}}\right)=0, \text { for all } k \text { and some } i_{0} \text {, then } \\
\Delta \tilde{\Omega}^{\text {Floer }}(\gamma)=0
\end{gathered}
$$

Therefore, Proposition 5.45 motivates us to define the wall of marginal stability for $\tilde{\Omega}^{\text {Floer }}$ in the following way:

Definition 5.46. For $\gamma_{1}, \gamma_{2}$ primitive, we define

$$
\begin{array}{r}
W_{\gamma_{1}, \gamma_{2}}=\left\{u \in B_{0} \mid \operatorname{Arg} Z_{\gamma_{1}}=\operatorname{Arg} Z_{\gamma_{2}}, \tilde{\Omega}^{\text {Floer }}\left(k_{1} \gamma_{1}\right) \tilde{\Omega}^{\text {Floer }}\left(k_{1} \gamma_{2}\right) \neq 0\right. \\
\text { for some } \left.k_{1}, k_{2} \in \mathbb{N}\right\} .
\end{array}
$$

The wall of marginal stability of $\gamma$ for holomorphic discs counting is given by

$$
W_{\gamma}=\bigcup_{\gamma=k \gamma_{1}+l \gamma_{2}} W_{\gamma_{1}, \gamma_{2}}
$$

It is easy to see that $W_{\gamma}$ is an open subset of $W_{\gamma}^{\prime}$ and $W_{\gamma}^{\prime \prime}$. The following theorem follows from similar argument in Lemma 5.31.

Theorem 5.47. Assume $[\partial \gamma] \neq 0$ and $\tilde{\Omega}^{\text {Floer }}\left(\gamma ; u_{0}\right)$ is well-defined, then $\tilde{\Omega}^{\text {Floer }}(\gamma ; u)$ is well-defined and locally a constant around $u_{0}$.

In the definition of the invariant $\tilde{\Omega}^{\text {Floer }}$, there is a choice of Ricci-flat metric to form the twistor family. However, we have 
Corollary 5.48. Assume $\omega, \omega^{\prime}$ are Ricci-flat metrics such that the corresponding invariants $\tilde{\Omega}(\gamma ; u)$ and $\tilde{\Omega}^{\prime}(\gamma ; u)$ are well-defined. Then

$$
\tilde{\Omega}(\gamma ; u)=\tilde{\Omega}^{\prime}(\gamma ; u)
$$

Proof. First we assume that $u \notin W_{\gamma}^{\prime \prime}$ then the proposition follows by similar cobordism argument as in Lemma 5.31. In particular, the proposition holds on an real analytic Zariski open subset of $B_{0}$. For $u \in W_{\gamma}^{\prime \prime} \backslash W_{\gamma}^{\prime}$, then by Theorem 5.47 there exists $u^{\prime} \notin W_{\gamma}^{\prime \prime}$ near $u$ such that

$$
\tilde{\Omega}(\gamma ; u)=\tilde{\Omega}\left(\gamma ; u^{\prime}\right)=\tilde{\Omega}^{\prime}\left(\gamma ; u^{\prime}\right)=\tilde{\Omega}^{\prime}(\gamma ; u)
$$

Lemma 5.49. Given a Lagrangian fibration $X \rightarrow B$ and $\delta>0$, then there exists $\epsilon(\delta)>0$ such that any holomorphic discs with non-trivial Lagrangian boundary condition with symplectic area less than $\delta$ should contained in an $\epsilon(\delta)$-neighborhood of a singular fibre.

Proof. This is a consequence of the gradient estimate of harmonic maps (ex. theorem 2.1 [39]). Indeed, assume a holomorphic disc with boundary on fibre $L$ and small area will fall in a neighborhood of $L$. However, a (topological) trivial $\mathbb{R}^{k} \times T^{l}$ fibration cannot bound any disc with non-trivial boundary homology class.

The following is another well-known folklore theorem. However, we cannot find the proof elsewhere. 
Theorem 5.50. Let $\gamma_{e}$ be the Lefschetz thimble, then

$$
\tilde{\Omega}^{\text {Floer }}\left(\gamma_{e} ; u\right)=1,
$$

for $u$ closed enough to a singularity of affine structure. Moreover, for $u$ close enough to the singularity, $\gamma_{e}$ is the class achieves minimum energy with $\tilde{\Omega}^{\text {Floer }}(\gamma) \neq 0$.

Proof. Assume $X$ is a K3 surface with special Lagrangian fibration around large complex limit. By Lemma 5.49, we will replace $X$ by the preimage of a $\epsilon$-neighborhood of singular point on the base, with the topology same as Ooguri-Vafa space. Notice that we still have Gromov's compactness theorem for $X$.

View the Ooguri-Vafa space as an elliptic fibration. Assume $\omega_{K 3}=$ $\omega_{O V}+i \partial \bar{\partial} \phi$, where $\phi$ is smooth.

Lemma 5.51. There is a path of hyperKähler triples $\left(\underline{X}, \omega_{t}, \Omega_{t}\right)$ connecting the restriction of $K 3$ and the Ooguri-Vafa space, keeping the elliptic fibration structure.

Proof. It suffices to prove that the uniqueness of complex Monge-Ampere equation with Dirichlet boundary condition and the solution is smoothly depends on the boundary condition. Take $u_{t}=t \phi$ and by the estimate in [19] there exists non-negative constant $\epsilon_{t}$, such that $\epsilon_{t}=0$ for $t \in\{0,1\}$, 
and $u_{t}$ are subsolutions of the below equation

$$
\left\{\begin{array}{l}
\left(\omega_{O V}+i \partial \bar{\partial} u_{t}\right)^{2}=\left(\frac{1}{2}-\epsilon_{t}\right) \Omega \wedge \bar{\Omega} \\
\left.u_{t}\right|_{\partial X}=t \phi
\end{array}\right.
$$

Therefore, Theorem 1.1 [15] provides a solution for the Dirichlet problem. To prove uniqueness, assume there are two solutions $\psi, \phi$ satisfy the complex Monge-Ampere equation with Dirichlet boundary condition. Then we have

$$
\operatorname{det}\left(g_{i \bar{j}}+\phi_{i \bar{j}}\right)=\operatorname{det}\left(g_{i \bar{j}}+\psi_{i \bar{j}}\right)
$$

or can rewrite it as

$$
\operatorname{det}\left(g_{i \bar{j}}+\phi_{i \bar{j}}+\left(\psi_{i \bar{j}}-\phi_{i \bar{j}}\right)\right) \operatorname{det}\left(g_{i \bar{j}}+\phi_{i \bar{j}}\right)^{-1}=1
$$

By arithmetic-geometric mean inequality,

$$
\frac{1}{2}\left[2+\Delta^{\prime}(\psi-\phi)\right] \geq 1
$$

where $\Delta^{\prime}$ is the Laplacian of metric $\left(g_{i \bar{j}}+\phi_{i \bar{j}}\right)$. Therefore, $\psi-\phi$ is subharmonic with respect to $\Delta^{\prime}$. Since $\psi$ and $\phi$ are smooth functions, we may add a constant and assume $\psi-\phi \geq 0$. Then

$$
0=\int_{X} \Delta^{\prime}(\psi-\phi)^{2}=2 \int_{X}(\psi-\phi) \Delta^{\prime}(\psi-\phi)+2 \int_{X}\left|\nabla^{\prime}(\psi-\phi)\right|^{2}
$$

All the terms arises from integration by part vanishes because $\psi$ and $\phi$ satisfy the same Dirichlet boundary condition. The first term of right hand 
side is non-negative implies $\nabla^{\prime}(\psi-\phi)=0$ or $\phi, \psi$ differs by a constant.

Assume the a special Lagrangian torus fibre $L$ in $\mathrm{K} 3$ bounds a holomorphic disc of class $\gamma$ with area smaller or equal to the Initial disc and has nontrivial invariants. We will use the continuity method to prove there is a disc with same property in the Ooguri-Vafa space. Indeed, notice that for Ooguri-Vafa space $\pi_{2}(X, L)$ is generated by the fibres and Initial discs. therefore wall of second kind doesn't contribute by Proposition 5.40. If wall of third kind contribute, then it degenerates to holomorphic discs in class $\gamma$ with area smaller or equal to $\left|Z\left(\gamma_{e}\right)\right|$ on $L_{t}$ such that $\Omega(\gamma) \neq 0$ and is indecomposable (thus is well-defined). Then there exists a neighborhood of $L_{t}$ bound this discs by Gromov compactness theorem. Again by Gromov-compactness theorem, there will be some disc with area smaller or equal to $\left|Z\left(\gamma_{e}\right)\right|$ and converge to the unique holomorphic disc in the OoguriVafa space. It contradicts to uniqueness of deformation of the only disc in Ooguri-Vafa space by hyperKähler rotation trick. Therefore, there are no holomorphic discs with area smaller than $\gamma_{e}$ and there is only a unique holomorphic disc represent $\gamma_{e}$. The theorem follows from $\mathcal{M}_{0, \gamma_{e}}(\mathfrak{X}, L)$ is Fredholm regular and consists just a point. Indeed, the zero of each branch of perturbed multi-section is isomorphic to $\left(W_{\alpha}, \omega_{\alpha}\right)$ and thus the invariant is by definition

$$
\int_{W_{\alpha}} \omega_{\alpha}=1
$$




\subsection{Multiple Cover Formula}

To prove the corresponding theorem, one ingredient is multiple cover formula of the holomorphic discs discussed in Theorem 5.50. Use argument similar to Theorem 5.50, it suffices to compute the multiple cover contribution from the following local model below: $X=T^{*} \mathbb{P}^{1}$ and $L \cong \mathbb{R}^{1} \times S^{1}$ is the fixed locus of an anti-symplectic, anti-holomorphic involution $\iota$. The author learn the localization technique from Chiu-Chu Melissa Liu. Using localization to compute multiple cover of discs invariants can be first found in [25][21], and later carry out rigorously in [33].

We first construct an special Lagrangian fibration with respect to IguchiHanson metric with $L$ one of the fibre. Let $X=T \mathbb{P}^{1}$ be the blow-up of $\mathbb{C}^{2} / \mathbb{Z}_{2}$ at the origin. Let $(y, \lambda),(x, \mu)$ be the coordinate chart on $X$, where $x=1 / y$ and $\mu=\lambda y^{2}$. Then there is an natural $S^{1}$-action on $X$ preserving $\lambda y=\mu x$. The Iguchi-Hanson metric $\omega_{E H}$ is invariant under this $S^{1}$-action and thus

$$
\begin{gathered}
X \longrightarrow \mathbb{R}^{2} \\
(y, \lambda) \mapsto\left(\mu_{S^{1}}, \operatorname{Re}(\lambda y)\right)
\end{gathered}
$$

is a special Lagrangian fibration with smooth fibres homeomorphic to $\mathbb{R} \times S^{1}$ [14]. Let $\iota:(y, \lambda) \mapsto(\bar{y}, \bar{\lambda})$ be an anti-holomorphic involution and antisymplectic involution. The fixed locus of $\iota$ is a special Lagrangian fibre $L$. Moreover, by maximal principle there exists a unique simple holomorphic disc (up to reflection $\iota$ ) in $X$ with boundary on $L$. This is a local model near the singularity of Ooguri-Vafa space. Locally around the singularity of the fibration, Iguchi-Hanson metric and Ooguri-Vafa metric are both $S^{1}$ 
invariant and thus arise from Gibbons-Hawking ansatz (section 2.6 [19]). Therefore, there is a family of hyperKähler triple from Gibbons-Hawking ansatz connecting these two spaces. Same argument as Remark 2.11, there is a unique embedded holomorphic discs in each space. Therefore, pseudoisotopy of Kuranishi structure along this family guarantees we can just compute the multiple cover formula for moduli space $\mathcal{M}_{d}=\mathcal{M}_{d \gamma}(\mathfrak{X}, L)$ of discs in $X=T^{*} \mathbb{P}^{1}$ (with $S^{1}$-family of complex structures by hyperKähler rotation) with boundary on $L$ and image only mapping to certain side of the equator.

Let $\gamma$ be the unique (up to sign) relative class bounding simple holomorphic disc and $\tilde{\gamma}$ is the homology class of zero section $\mathbb{P}^{1}$ in $X$. Since the invariant $\tilde{\Omega}^{\text {Floer }}(d \gamma)$ is independent of the choice of Kuranishi structures, we will choose the Kuranishi structure for computation purpose as follows: The obstruction bundle $F_{d}$ is an orbi-bundle over an orbifold $M_{d}$ and the Kuranishi map is just the zero section. For each point $[f] \in \mathcal{M}_{d}$, it has a corresponding point in $M_{d}$ again denoted by $[f]$ and

$$
\left.F_{d}\right|_{[f]}=H^{1}\left(D^{2}, \partial D^{2} ; f^{*} T X, f^{*} T L\right) / \mathbb{R},
$$

where the quotient $\mathbb{R}$-factor is induced by the $S^{1}$-family of complex structures by hyperKähler rotation. Also the tangent space of $M_{d}$ at $[f]$ is

$$
\left.T M_{d}\right|_{[f]}=H^{0}\left(D^{2}, \partial D^{2} ; f^{*} T X, f^{*} T L\right) / A u t\left(D^{2}\right) .
$$

Notice that $\mathcal{M}_{d}$ is bijective with the moduli space $\tilde{\mathcal{M}}_{d}$ of real rational curves 
of degree $d$ in a twistor family of $X$. (Notice that this point is quite different from the situation of [33]) Therefore, we double the Kuranishi structure on $\mathcal{M}_{d}$ and equip $\tilde{\mathcal{M}}_{d}$ with an $\iota$-equivariant Kuranishi structure under this identification. We may choose the perturbed multi-section on $\tilde{M}_{d}$ to be $\iota$-invariant. Therefore,

$$
\begin{aligned}
\tilde{\Omega}^{\text {Floer }}(d \gamma): & =\operatorname{Corr}_{*}\left(\mathcal{M}_{d} ; \text { tri } i, t r i\right)(1) \\
& =\frac{1}{2} \operatorname{Corr}_{*}\left(\tilde{\mathcal{M}}_{d} ; \text { tri }, \text { tri }\right)(1)
\end{aligned}
$$

The Kuranishi structure on $\tilde{\mathcal{M}}_{d}$ is a smooth closed orbifold $\tilde{M}_{d}$ of real dimension $2 d-2$ with an orbibundle $\tilde{F}_{d}$ and Kuranishi map is the zero section. In particular, (41) is just the top Chern class of the orbibundle $\tilde{F}_{d}$. We will use localization to compute this top Chern class. Using the fact that $L$ is the fixed locus of $\iota$, which is both anti-holomorphic and anti-symplectic, one concludes that maps in $\mathcal{M}_{d}$ is a $d$-fold cover from trees of disks to the zero section $\mathbb{P}^{1}$ with boundary in $S^{1}$. Then there is only two torus fixed point in the moduli space $\tilde{\mathcal{M}}_{d}$, namely, the doubling of $f_{d}$ given by $z \mapsto z^{d}$ composed with the only embedded disc (we will again denote it by $f_{d}$ ) and its reflection under the involution $\iota$.

Let $(a)$ denote the complex line with the $U(1)$-action of weight $a$; while $(0)_{\mathbb{R}}$ denotes the real line with the trivial $\mathrm{U}(1)$-action. It is easy to see that the doubling of bundle

$$
\left(f_{d}^{*} T X, f_{d}^{*} T L\right)_{\mathbb{C}} \cong \mathcal{O}_{\mathbb{P}^{1}}(-2) .
$$


Then straightforward computation shows

$$
\begin{array}{lr}
\operatorname{Aut}\left(D^{2}\right)=(1 / d)+(0)_{\mathbb{R}}, & (\text { real dimension } 3) \\
H^{0}\left(D^{2}, \partial D^{2} ; f_{d}^{*} T X, f_{d}^{*} T L\right)=\oplus_{j=1}^{d}(j / d)+(0)_{\mathbb{R}} . & (\text { real dimension } 2 d+1)
\end{array}
$$

So together we have

$$
\left.T \tilde{M}_{d}\right|_{\left[f_{d}\right]}=\oplus_{j=2}^{d}(j / d) .
$$

On the other hand,

$H^{1}\left(D^{2}, \partial D^{2} ; f_{d}^{*} T X, f_{d}^{*} T L\right)=\oplus_{j=1}^{d-1}(-j / d)+(0)_{\mathbb{R}} \quad($ real dimension $2 d-1)$.

So the fibre of obstruction bundle $\tilde{F}_{d}$ at $f_{d}$ is given by

$$
\left.\tilde{F}_{d}\right|_{\left[f_{d}\right]}=\oplus_{j=1}^{d-1}(-j / d)
$$

because the counting in $S^{1}$-family is equivalent to changing the obstruction bundle by $(0)_{\mathbb{R}}$. Finally we get

$$
\tilde{\Omega}^{\text {Floer }}(d \gamma)=\frac{1}{2} \int_{\tilde{M}_{d}} e_{U(1)}\left(\tilde{F}_{d}\right)=\frac{1}{\left|A u t\left(f_{d}\right)\right|} \frac{e_{U(1)}\left(\left.\tilde{F}_{d}\right|_{\left[f_{d}\right]}\right)}{e_{U(1)}\left(\left.T \tilde{M}_{d}\right|_{\left[f_{d}\right]}\right)}=\frac{(-1)^{d-1}}{d^{2}}
$$

which coincides with the multiple cover formula of $\tilde{\Omega}^{\text {trop }}$ for initial discs (17).

To sum up, we prove the multiple cover formula for initial discs:

Theorem 5.52. Let $\gamma$ be the class of initial disc around $I_{1}$-type singular 
fibre, then

$$
\tilde{\Omega}^{\text {Floer }}(d \gamma)=\frac{(-1)^{d-1}}{d^{2}} \text {, for every } d \in \mathbb{N} \text {. }
$$

Remark 5.53. It is not enough to show that the initial data $\Omega(\gamma, u)=1$ for $u$ near the singularity from the observation that there is a unique simple disc. More than that, one also needs a correct multiple cover formula Theorem 5.52.

Remark 5.54. For general $I_{n}$ type singular fibres, the local model can also be described by Gibbons-Hawking ansatz and same argument of 5.50 provides that initial data $\tilde{\Omega}^{\text {Floer }}\left(d \gamma_{e}\right)=\frac{n(-1)^{d-1}}{d^{2}}$. The rest of invariants are provided by wall-crossing formula, thus discussion can also generalized to elliptic K3 surfaces with $I_{n}$-type singularities.

\subsection{Corresponding Theorem}

Similar argument in Theorem 5.50 together with induction, there exists $\delta>0$ such that any holomorphic disc with boundary on torus fibre $L$ and with symplectic area less than $\delta$ implies $L$ falls in a certain neighborhood of a singular fibre. In other words, the holomorphic disc is a multiple cover of the Lefschetz thimble which realized the minimal area among relative classes with non-trivial invariant. Now we want to prove that all other holomorphic discs in this $S^{1}$-family are all from "scattering"- gluing of these initial discs.

Assume $\gamma_{u} \in H_{2}\left(X, L_{u}\right)$ is represented as a holomorphic disc with boundary on $L_{u}$ such that $\tilde{\Omega}^{\text {Floer }}(\gamma ; u) \neq 0$. Without lose of generality, we may assume $\int_{\partial \gamma_{u}} \Omega \in \mathbb{R}_{+}$. There is an affine half line $l$ emanating from $y$ on 
the base such that $\int_{\partial \gamma_{t}} \Omega$ is a decreasing function of $t \in l$, where $\partial \gamma_{t}$ is the parallel transport of $\partial \gamma_{u}$ along $l$. Since $\int_{\partial \gamma_{t}} \Omega$ is an affine function along $l$, it has no lower bound. There is some point $u^{\prime} \in l$ such that $\int_{\partial \gamma_{u^{\prime}}} \Omega=0$. Thus, there are two cases:

1. If $\tilde{\Omega}^{\text {Floer }}(\gamma ; u)=\tilde{\Omega}^{\text {Floer }}\left(\gamma ; u^{\prime}\right)$ then $L_{u^{\prime}}$ is a singular fibre by Lemma 5.49. In particular, if $L_{u^{\prime}}$ is of $I_{n}$-type singular fibre then $\gamma_{u^{\prime}}$ is represented by multiple cover of the unique area minimizing holomorphic disc and $\partial \gamma_{u}$ is the parallel of $\gamma_{u^{\prime}}$ along $l$.

2. If $\tilde{\Omega}^{\text {Floer }}(\gamma ; u) \neq \tilde{\Omega}^{\text {Floer }}\left(\gamma ; u^{\prime}\right)$ then from the Proposition 5.45 and Theorem 5.36, there exists $\gamma_{n, u^{\prime}}$ in the same phase with $\gamma_{u^{\prime}}$ such that $\sum_{n} \gamma_{n, u^{\prime}}=\gamma_{u^{\prime}}$ and $\tilde{\Omega}^{\text {Floer }}\left(\gamma_{n} ; u^{\prime}\right) \neq 0$. Assume the boundary is of second kind. By maximal principle, it happens only when $\gamma_{1, u^{\prime}}$ is a multiple of $\gamma_{2, u^{\prime}}$. Then by Proposition 5.40, this does not contribute to $m_{-1, \gamma}$. If the boundary is of third kind, the theorem is followed by applying the Stokes theorem and Proposition 5.40. The sign is given by Proposition 3.15. Then we replace $\gamma$ by $\gamma_{i, u^{\prime}}$ and repeat the same processes. The procedure will stop at finite time because of Gromov compactness theorem. By induction, every holomorphic disc with nontrivial invariant give rise to a tropical disc, which is formed by the union of the affine segments on the base. In particular, the balancing conditions are guaranteed by the conservation of charges $\sum_{n} \gamma_{n, u^{\prime}}=\gamma_{u^{\prime}}$ at each vertex $u^{\prime}$.

To sum up, we proved the following theorem by this attractor flow mechanism [6] of holomorphic discs. 
Theorem 5.55. Let $X$ be an elliptic K3 surface (singular fibres not necessarily of $I_{n}$-type). For every relative class $\gamma \in H_{2}\left(X, L_{u}\right)$ with $\tilde{\Omega}^{\text {Floer }}(\gamma ; u) \neq$ 0 has a corresponding tropical disc $\phi$ such that $[\phi]=\gamma$. Moreover, the symplectic area of the holomorphic disc is just the total affine length of the corresponding tropical disc.

Below we will demonstrate a non-trivial example of wall-crossing phenomenon of invariants $\tilde{\Omega}^{\text {Floer }}(\gamma ; u)$.

Example 5.56. Assume there are two initial rays emanating from two $I_{1}$ type singularities of phase $\vartheta_{0}$ intersect at $p \in B_{0}$. From Theorem 5.10, there are two initial holomorphic discs of relative classes $\gamma_{1}, \gamma_{2}$ corresponding to the initial rays which are Fredholm. Moreover, the local model provided in the proof of Theorem 5.10 indicates that they intersect transversally in $L_{p}$. From automatic transversality of K3 surfaces, these two discs cannot be smoothed out in $L_{p}$. To prove that these two discs will smooth when changing the Lagrangian boundary condition, First pick two point $p_{1}, p_{2}$ near $p$ but on the different side of wall of marginal stability $W_{\gamma_{1}, \gamma_{2}}$. Let $\psi:(-\epsilon, 1+\epsilon)$ be a path on $B_{0}$ such that $\psi(0)=p_{1}, \psi(1)=p_{2}$ and intersect $W_{\gamma_{1}, \gamma_{2}}$ once transversally at $p$. Recall $\mathcal{X}$ is the total space of twistor space of $X$ with two fibres with elliptic fibration threw away. Then $L_{u} \times S_{\vartheta}^{1}$ is a totally real torus in $\mathcal{X}$. Now consider an complex manifold $\mathfrak{X} \times \mathbb{C}$ with a totally real submanifold

$$
\mathcal{L}=\bigcup_{t}\left(L_{\psi(t)} \times S_{\vartheta}^{1}\right)
$$

By our assumption, there are two regular holomorphic discs in $\mathcal{X}$ with bound- 
aries in $L_{p} \times\left\{\vartheta_{0}\right\} \subseteq \mathcal{L}$ of relative classes again we denoted by $\gamma_{1}, \gamma_{2}$. The tangent of evaluation maps for both discs are two dimensional and transversal. By Theorem 4.1.2 [2], these two discs can be smoothed out into simple regular discs in $\mathcal{L}$ and the union of initial holomorhpic discs is indeed the codimension one of the boundary of the usual moduli space of holomorphic discs $\mathcal{M}_{0, \gamma_{1}+\gamma_{2}}(\mathcal{X}, \mathcal{L})$ which exists by Remark 5.29. By maximal principle twice, each of the holomorphic disc falls in $\mathcal{M}_{\gamma_{1}+\gamma_{2}}\left(\mathfrak{X}, L_{\psi(t)}\right)$ for some $t$. In particular,

$$
\mathcal{M}_{1, \gamma_{1}}\left(\mathfrak{X}, L_{p}\right) \times_{L \times S_{\vartheta}^{1}} \mathcal{M}_{1, \gamma_{2}}\left(\mathfrak{X}, L_{p}\right) \subseteq \mathcal{M}_{\gamma_{1}+\gamma_{2}}\left(\mathfrak{X},\left\{L_{t}\right\}\right)
$$

as codimension one boundary. Therefore,

$$
\begin{aligned}
\Delta \tilde{\Omega}^{\text {Floer }}\left(\gamma_{1}+\gamma_{2}\right) & = \pm \operatorname{Corr}_{*}\left(\mathcal{M}_{1, \gamma_{1}}\left(\mathfrak{X}, L_{p}\right) \times_{L \times S_{\vartheta}^{1}} \mathcal{M}_{1, \gamma_{2}}\left(\mathfrak{X}, L_{p}\right) ; \text { tri,tri }\right)(1) \\
& =\left|\left\langle\gamma_{1}, \gamma_{2}\right\rangle\right| \tilde{\Omega}^{\text {Floer }}\left(\gamma_{1}, p\right) \tilde{\Omega}^{\text {Floer }}\left(\gamma_{2}, p\right),
\end{aligned}
$$

by Theorem 5.10, Proposition A.14 and Proposition 5.40. Assume moreover, that the two $I_{1}$-type singularities on the base are closed enough to each other. Using the same argument in the proof of Theorem 5.55, the difference of the invariant appear the side of the wall of marginal stability satisfying (3.15) and thus determined the sign.

Notice that the jump of the invariant is exactly the same as described in Theorem 4.6. We expect the wall-crossing formula (15) also holds for $\tilde{\Omega}^{\text {Floer }}(\gamma ; u)$. Together with Theorem 5.52, it will imply the following corresponding of counting holomorphic discs and tropical discs: 
Conjecture 5.57. For elliptic K3 surface with $24 I_{1}$-type singular fibres, $\tilde{\Omega}^{\text {Floer }}(\gamma ; u)=\tilde{\Omega}^{\text {trop }}(\gamma ; u)$ for every $u \in B_{0}$. In particular, the open GromovWitten invariant $\tilde{\Omega}^{\text {Floer }}(\gamma ; u) \in \mathbb{Q}$.

Therefore, Conjecture 5.57 and Theorem 5.52 together provide an algorithm to compute all the invariants $\tilde{\Omega}^{\text {Floer }}(\gamma, u)$ on elliptic K3 surfaces with $24 I_{1}$-type singular fibres. For more explicit computation of the invariants $\tilde{\Omega}^{\text {Floer }}$, one need to study the affine geometry induced by elliptic fibration to describe the position of possible walls of marginal stability. The explicit jump from wall-crossing formula may be computed from the study of quiver representation moduli spaces [34] or proving the generating functions of these invariants satisfy certain modularity. We close this section with following conjecture which indicates the existence of a Gopakumar-Vafa type invariants $\Omega^{\text {Floer }}$.

Conjecture 5.58. There exists $\left\{\Omega^{\text {Floer }}(\gamma ; u) \in \mathbb{Z} \mid \gamma \in H_{2}\left(X, L_{u}\right)\right\}$ such that

$$
\tilde{\Omega}^{\text {Floer }}(\gamma ; u)=\sum_{d>0} \pm d^{-2} \Omega^{\text {Floer }}(\gamma / d ; u)
$$

\subsection{Other Local Models}

The proof of Theorem 5.55 doesn't depend on the type of singular fibre we have. However, different kind of singular fibres will impose different initial data instead of (17).

Example 5.59. ( $I_{0}^{*}$-type singular fibre) Let $E_{\tau_{i}}$ be elliptic curves for $i=1,2$ and Let $X_{0}$ be the quotient of $E_{\tau_{1}} \times E_{\tau_{2}}$ by the involution $\iota: z_{i} \mapsto-z_{i}$. Let $X$ be the blow-up of $X_{0}$ at the 16 ordinary double points and $X$ is called the 
Kummer K3 surface. The Kummer K3 surface admits an elliptic fibration with 4 singular fibres of $I_{0}^{*}$-type. Since the elliptic fibration is isotrivial, the $S^{1}$-family of affine structures reduce to the same one, which coincides with the $\mathbb{Z}_{2}$-quotient of the standard affine structure on elliptic curve $E_{\tau_{1}}$.

It is pointed out in [9] that given a pair $(k, l) \in \mathbb{Q}^{2}$, consider a map $f:\{|s|<1\} \times \mathbb{R}_{t} \rightarrow\left(X_{0}, E_{(k, l)}\right)$ by

$$
z_{1}=(k+i l) s, \quad z_{2}=(k+i l) t
$$

When $\|(k, l)\| \ll 1$, then the image of $f$ will falls in a small neighborhood of the $I_{0}^{*}$-type singular fibre. It is straight forward to see that the map $f$ lifts to $X$ and the image is still a smooth disc. Moreover the pull-back of Eguchi-Hanson metric $\omega$ and $\operatorname{Im} \Omega$ is zero. Therefore, by the similar technique of deformation of special Lagrangian discs and estimates in [27], it can be deformed to a smooth special Lagrangian discs with respect to true Ricci-flat metric and thus gives rise to a holomorphic disc from hyperKähler rotation trick. In particular, they become regular holomorphic discs after hyperKähler rotation. We expect these are all the holomorphic discs near the $I_{0}^{*}$-type singular fibre. 


\section{Quantum Corrected SYZ Transform on the Ooguri-Vafa Space}

Given a torus bundle $X \rightarrow B$ and its dual $\check{X} \rightarrow B$, one can defined the SYZ transform as follows:

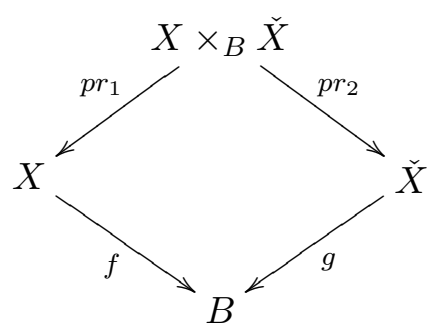

For a given differential form $\alpha$ on $X$, we define the SYZ transform by

$$
\begin{aligned}
\mathcal{F}_{F}: & \mathcal{A}^{*}(X) \rightarrow \mathcal{A}^{*}(\check{X}) \\
\alpha & \mapsto r_{2 *}\left(p r_{1}^{*} \alpha \wedge F\right),
\end{aligned}
$$

where $F$ is a differential form on $X \times_{B} \check{X}$, and $p r_{1 *}$ denotes integration along fibres.

In [?CL] it is proved that SYZ transform exchange the toric symplectic form and holomorphic volume form for toric manifolds if $F$ is the curvature of the Poincare bundle. It is pointed out in [4], if we choose the same $F$ for the Ooguri-Vafa space, SYZ transform only exchange the semi-flat symplectic form and holomorphic volume form. 


$$
\begin{aligned}
\Omega(\zeta) & =\frac{-1}{4 \pi^{2} R}\left(2 \pi R \operatorname{Re}(\bar{\zeta} y)+i d \theta_{e}\right) \wedge\left(i d \theta_{m}+2 \pi i A-2 \pi V \operatorname{Im}(\bar{\zeta} y)\right) \\
\check{\omega}(-i \zeta) & =-d \operatorname{Re}(\bar{\zeta} y) \wedge\left(\frac{d \check{\theta}_{m}}{2 \pi}+\check{A}\right)-\check{V} \operatorname{Im}(\bar{\zeta} y) \wedge \frac{d \check{\theta}_{e}}{2 \pi R},
\end{aligned}
$$

where

$$
\begin{aligned}
V & =V^{s f}+V^{i n s t} \\
V^{s f} & =-\frac{R}{4 \pi}(\log y+\log \bar{y}) \\
V^{i n s t} & =\frac{R}{2 \pi} \sum_{n \neq 0} e^{i n \theta_{e}} K_{0}(2 \pi R|n y|)
\end{aligned}
$$

and

$$
\begin{aligned}
A & =A^{s f}+A^{i n s t} \\
A^{s f} & =\frac{i}{8 \pi^{2}}(\log y-\log \bar{y}) d \theta_{e} \\
A^{i n s t} & =-\frac{R}{4 \pi}\left(\frac{d y}{y}-\frac{d \bar{y}}{\bar{y}}\right) \sum_{n \neq 0}(\operatorname{sgn} n) e^{i n \theta_{e}}|y| K_{1}(2 \pi R|n y|) .
\end{aligned}
$$

All the instanton correction are excluded because they are higher mode in Fourier expansion. However, from the formal construction of the holomorphic volume form of twistor family, we have a more natural candidate of $F$ as follow:

$$
\begin{gathered}
d \Theta(\zeta)=d \theta_{m}+\pi i V^{i n s t}\left(\frac{1}{\zeta} d y-\zeta d \bar{y}\right)+2 \pi A^{i n s t}+\Delta(\zeta) \\
d \check{\Theta}(-i \zeta)=d \check{\theta}_{m}+\pi i \check{V}^{i n s t}\left(\frac{1}{\zeta} d y+\zeta d \bar{y}\right)+2 \pi \check{A}^{i n s t}+\check{\Delta}(-i \zeta)
\end{gathered}
$$


and

$$
F=i \frac{d \Theta_{m}(\zeta)}{2 \pi} \wedge \frac{d \check{\theta}_{e}}{2 \pi R}-\frac{d \theta_{e}}{2 \pi R} \wedge \frac{d \check{\Theta}_{m}(-i \zeta)}{2 \pi}
$$

The quantum corrected SYZ transform is as follows:

$$
\begin{aligned}
& \mathcal{F}\left(e^{i \check{\omega}(-i \zeta)}\right) \\
= & \int_{\check{L}} e^{i \check{\omega}(-i \zeta)} \wedge e^{F} \\
= & \int_{\check{L}} e^{i\left[-d \operatorname{Re}(\bar{\zeta} y) \wedge \check{\theta}_{0}-\check{V} d \operatorname{Im}(\bar{\zeta} y) \wedge \frac{d \check{\theta}_{e}}{2 \pi R}\right]+\left[-\frac{d \Theta_{m}(\zeta)}{2 \pi} \wedge \frac{d \check{\theta}_{e}}{2 \pi R}+\frac{d \theta_{e}}{2 \pi R} \wedge \frac{d \check{\Theta}_{m}(-i \zeta)}{2 \pi}\right]} \\
= & \int_{\check{L}} e^{-i\left(d \operatorname{Re}(\bar{\zeta} y)+\frac{i d \theta_{e}}{2 \pi R}\right) \wedge \check{\theta}_{0}+i\left(-\check{V} d \operatorname{Im}(\bar{\zeta})+i \frac{d \Theta_{m}(\zeta)}{2 \pi}+i A^{s f}\right) \wedge \frac{d \check{\theta}_{e}}{2 \pi R}} \\
= & \int_{\check{L}}-i \cdot i\left(d \operatorname{Re}(\bar{\zeta} y)+\frac{i d \theta_{e}}{2 \pi R}\right) \wedge \check{\theta}_{0} \wedge\left(-\check{V} d \operatorname{Im}(\zeta y)+i \frac{d \Theta_{m}(\zeta)}{2 \pi}+i A^{s f}\right) \wedge \frac{d \check{\theta}_{e}}{2 \pi R} \\
= & \int_{\check{L}}-i \cdot i\left(d \operatorname{Re}(\bar{\zeta} y)+\frac{i d \theta_{e}}{2 \pi R}\right) \wedge\left(-\check{V} d \operatorname{Im}(\zeta y)+i \frac{d \Theta_{m}(\zeta)}{2 \pi}+i A^{s f}\right) \wedge \frac{d \check{\theta}_{e}}{2 \pi R} \wedge \check{\theta}_{0} \\
= & \int_{\check{L}}\left(d \operatorname{Re}(\bar{\zeta} y)+\frac{i d \theta_{e}}{2 \pi R}\right) \wedge\left(-V d \operatorname{Im}(\zeta y)+i \frac{d \theta_{m}}{2 \pi}+i A\right) \wedge \frac{d \check{\theta}_{e}}{2 \pi R} \wedge \check{\theta}_{0}=\Omega(\zeta)
\end{aligned}
$$

The third equality is because $i \check{V}^{i n s t} \operatorname{Re}(\bar{\zeta} y)+\check{\Delta}(-i \zeta)$ are higher Fourier mode and

$$
\frac{d \theta_{e}}{2 \pi R} \wedge 2 \pi \check{A}^{s f}=-2 \pi A^{s f} \frac{d \check{\theta}_{e}}{2 \pi R} .
$$


The sixth equality is because

$$
\begin{aligned}
& -\check{V} d \operatorname{Im}(\zeta y)+\left(i \frac{d \Theta_{m}(\zeta)}{2 \pi}+i A^{s f}\right) \\
= & -\check{V} d \operatorname{Im}(\zeta y)+\frac{i d \theta_{m}}{2 \pi}-V^{i n s t} \operatorname{Im}(\bar{\zeta} y)+i A+\Delta(\zeta) \\
= & -V d \operatorname{Im}(\zeta y)+i \frac{d \theta_{m}}{2 \pi}+i A+\Delta(\zeta)
\end{aligned}
$$

and $\Delta(\zeta) \propto \frac{d \chi_{e}(\zeta)}{\chi_{e}(\zeta)}$ thus has no contribution.

To sum up, we proved SYZ transform indeed transform the symplectic form to the holomorphic 2-form of mirrors. The corrected curvature term encodes the reduced counting defined in Section 5. This is an example of Fourier-Mukai type transform in the level of differential forms with quantum correction.

\section{Real Noether-Lefschetz Theory and Open GW Invariants on Calabi-Yau 3-Folds}

Mathematicians focus much about the string duality between type IIA string and type IIB string theory which predicts mirror symmetry. However, there is another string duality between type IIA and heterotic string theory proposed by Karchu-Vafa which also provide interesting mathematical implications. This IIA-heterotic string duality suggests that any Calabi-Yau 3-folds with K3 fibration admits an heterotic string dual, which is a product of a K3 surface and an elliptic curve. It predicts that the counting of embedded rational curves in $X$ is related to the exponents of product expression of specific automorphic forms on the complex moduli of K3 surfaces. This is usually 
referred as the Harvey-Moore conjecture. It is first proved by Yau and Zaslow that the generating function of embedded rational curve countings is an modular form [40]. The well-known fact is that the moduli space of genus zero curves in a K3 surface has negative virtual dimension. Therefore, the stanford genus zero Gromov-Witten invariants vanishes on K3 surfaces. To interpret the counting of embedded rational curves in K3 surfaces in terms of Gromov-Witten theory, one has to introduce the reduced Gromov-Witten invariants by changing the usual tangent-obstruction theory [26][3][32]. Later Liu and Maulik-Pandharipande generalized the Harvey-Moore conjecture and proved that the Gromov-Witten invariants on Calabi-Yau 3-folds with K3 fibration can be derived from reduced Gromov-Witten invariants on K3 surfaces and Noethory-Lefschetz numbers. Here we will provide an open analogue of the genus zero result in [32].

\subsection{Real Noether-Lefschetz Theory}

From the discussion in section five, we know the only obstruction to deform holomorphic discs in K3 surfaces is given by the phase, which is homological. This motivate to consider the following real Noether-Lefschetz theory:

Let $\left(X_{0}, \omega_{X_{0}}, \Omega_{X_{0}}\right)$ be a $\mathrm{K} 3$ surface admits a special Lagrangian torus $L_{0}$. Let $\mathcal{M}$ be the moduli space of pairs of K3 surfaces and its oriented special Lagrangian submanifolds with homology class $\left[L_{0}\right]$. Then $\mathcal{M}$ can be identified as the $S^{2}$ bundle over an $S^{1}$-bundle over the moduli space of elliptic K3 surfaces such that the fibre homology class is $\left[L_{0}\right]$. The $S^{2}$ fibres corresponding to parametrization of special Lagrangian torus in a fixed K3 surface. The later $S^{1}$ fibres corresponding to the $S^{1}$-family of special 
Lagrangian fibred K3 surfaces induced from hyperKähler rotation if given elliptic K3 surfaces and their Kähler classes. Both the deformation of elliptic K3 surfaces and its special Lagrangian submanifolds are unobstructed, we know that $\mathcal{M}$ is a smooth manifold. The moduli space of elliptic K3 is complex thus admits a natural orientation. We use the right thumb rule to determine the orientation of $S^{1}$ fibres. The moduli space of special Lagrangian in side a fixed K3 surface has tangents spaced naturally identified as $\mathcal{H}^{1}\left(T^{2}\right)$ also admits a natural orientation from the orientation of special Lagrangian $T^{2}$. Since $\mathcal{M}$ is locally product of above two moduli spaces, thus is oriented. Given a charge $\gamma \in H_{2}\left(X_{0}, L_{0}\right)$ such that $\tilde{\Omega}(\gamma) \neq 0$, there is a submersion from a local chart $\mathfrak{U}$ of $\mathcal{M}$ containing $\left(X_{0}, L_{0}\right)$ to $S_{\vartheta}^{1}$,

$$
\operatorname{Arg}_{\gamma}=\operatorname{Arg}\left(Z_{\gamma}\right), \text { where } Z_{\gamma}(X, L)=\int_{\gamma_{(X, L)}} \omega_{X}+i \operatorname{Im} \Omega_{X}
$$

The fibre of $\operatorname{Arg}_{\gamma}$ over $0 \in S^{1}$ is denoted by $N L_{\gamma}$ can be viewed as analogue of the Noether-Lefschetz divisor. Indeed, the central charge $Z_{\gamma}$ is holomorphic and regular from the from (2.14). Therefore, $\mathrm{Arg}_{\gamma}$ is regular by open mapping theorem and $N L_{\gamma}$ is a locally a smooth manifold. We will call them Noether-Lefschetz walls because they are codimension one submanifolds. Since the phase $S_{\vartheta}^{1}$ is viewed as the unit circle in the complex plane and thus is naturally oriented, the Noether-Lefschetz walls $N L_{\gamma}$ are also oriented.

Let $\phi: S^{1} \rightarrow \mathcal{M}$ be a smooth immersion and $\phi\left(S^{1}\right)$ intersects $N L_{\gamma}$ only finitely many times. Assume $\phi(t) \in N L_{\gamma}$. We may define the real 
Noether-Lefschetz numbers as follows:

$$
N L_{\gamma}^{\phi, t}=\phi(t-\epsilon, t+\epsilon) \cdot N L_{\gamma}
$$

where $\epsilon$ is a positive number small enough. Notice that each term in the summation only achieves values either \pm 1 or 0 .

\subsection{Open Gromov-Witten Invariants on Calabi-Yau 3-Folds with K3 Fibration}

Let $\pi: X \rightarrow C$ be a Calabi-Yau 3-fold with K3 fibration. Let $\mathcal{M}_{X}$ to be those pairs appears in $X$. Let $\phi: S^{1} \rightarrow \mathcal{M}_{X}$ such that $L=\bigcup_{t} L_{t}$ is a smooth 3 -tori, where $L_{t}$ is the projection of $\phi(t)$ to the second factor. The case when $L$ is not a 3 -torus should followed similar argument and computation but might with involved signs.

Proposition 7.1. With the notation above, $L$ is a totally real submanifold of $X$ and the fibral relative class in $H_{2}(X, L)$ will have Maslov index zero. Proof. Assume $J T L \cap T L \neq\{0\}$. We may assume there are $v_{1}, v_{2} \in T L_{t}$ and $v_{3}, v_{4} \in T L$ and projects to non-zero vector on $T C$ such that

$$
J\left(v_{1}+v_{3}\right)=v_{2}+v_{4} \quad \text { or } \quad J v_{3}-v_{4}=v_{2}-J v_{1} .
$$

However, the right hand side projects nonzero to $T C$ while the left hand side does not. The second statement follows directly from the fact that $L$ is fibred by special Lagrangian torus.

Since $L$ has trivial tangent bundle we can associate it with the trivial 
spin structure. Although $L$ is only totally real, we don't have the notion of symplectic area for general $J$-holomorphic discs. However, if we only restrict to the vertical relative classes respect to the pair $(X, L)$, the symplectic area is still well-defined and Gromov compactness for pair $(X, L)$ still holds. One can construct of Kuranishi structure in [12], one has

Theorem 7.2. [12] Given a fibral class $\gamma$ with $\partial \gamma \neq 0$, there exists Kuranishi structure and perturbed multi-sections such that

$$
\partial \mathcal{M}_{0, \gamma}(\mathfrak{X}, L)=\cup \bigcup_{\gamma_{1}+\gamma_{2}=\gamma}\left(\mathcal{M}_{1, \gamma_{1}}(X, L)_{e v_{0}} \times_{e v_{0}} \mathcal{M}_{1, \gamma_{2}}(X, L)\right) / \mathbb{Z}_{2}
$$

the restriction of the Kuranishi structure and multi-sections on the left hand side coincides with the fibre product of those from the right hand side.

Notice that $\gamma_{i}$ are all of fibral class. Notice that the wall of marginal stability discussed in previous chapters now become real codimension two in $\mathcal{M}$. Indeed, $W_{\gamma_{1}, \gamma_{2}}$ is the transverse intersection of $N L_{\gamma_{1}}$ and $N L_{\gamma_{2}}$. Therefore, given a totally real torus from a generic choice of $\phi$ the moduli space of holomorphic $\operatorname{discs} \mathcal{M}_{0, \gamma}(X, L) \leq \lambda$ with $\left|Z_{\gamma}\right| \leq \lambda$ has no codimension one boundary components, we can define the open Gromov-Witten invariant associated to $\gamma$,

$$
G W_{\gamma}^{\leq \lambda}(X, L)=\operatorname{Corr}_{*}\left(\mathcal{M}_{0, \gamma}(X, L)^{\leq \lambda} ; \text { tri, tri }\right)(1)
$$

From the assumption and Proposition $4.1[8], G W_{\gamma}(X, L)$ is well-defined.

Remark 7.3. If $L \cap X_{t}=L_{t}$ then $H_{2}\left(X_{t}, L_{t}\right) \subseteq H_{2}(X, L)$ and we can omit the subindex $\leq \lambda$. 


\subsection{Comparison of the Invariants for K3 and Calabi-Yau 3- Folds}

The walls of marginal stability described in section 5 are codimension two in $\mathcal{M}_{X}$. We assume $\phi\left(S^{1}\right)$ is in generic position, namely,

1. $\phi\left(S^{1}\right)$ avoids the walls of marginal stability of energy less than $\lambda$

2. $\phi\left(S^{1}\right)$ intersect $N L_{\gamma}$ only finitely many times, for $\gamma$ with energy less than $\lambda$.

Then the two moduli space of $\operatorname{disc} \mathcal{M}_{0, \gamma}\left(\mathfrak{X}_{t}, L_{t}\right)$ and

$$
\mathcal{M}_{0, \gamma}^{t}(X, L)=\left\{f:\left(D^{2}, \partial D^{2}\right) \rightarrow(X, L) \in \mathcal{M}_{0, \gamma}(X, L) \mid \operatorname{Im}(f) \subseteq L_{t}\right\}
$$

for $\gamma$ such that $\left|Z_{\gamma}\right|<\lambda$, are the same as topological space and has no codimension one boundary. In particular, the disc invariant for classes with energy less than $\lambda$ on both K3 surfaces and Calabi-Yau 3-folds are well-defined. We first assume that the image of $\phi$ intersect the Noether-Lefschetz wall $N L_{\gamma}$ transversally. Then $H^{0}\left(f^{*} N_{X_{t} / X}, f^{*} N_{L_{t} / L}\right) \cong \mathbb{R}$ induces a nontrivial element in $H^{1}\left(f^{\prime *} T_{X_{t}}, f^{* *} T_{L_{t}}\right)$. Moreover, we have the following commute diagram: 


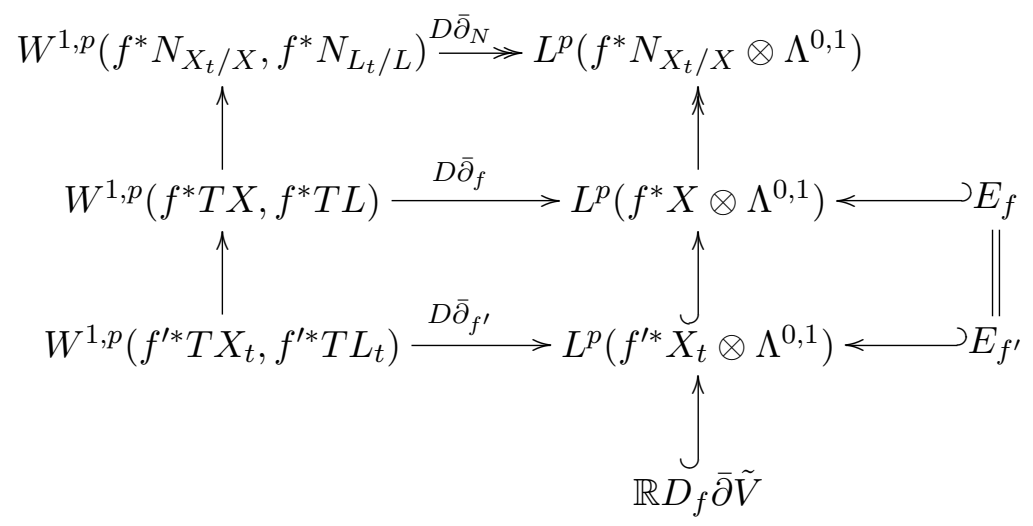

Remark 7.4. Using the long exact sequence associate to the middle two column of above diagram and the fact that $X_{t} \subseteq X$ is a complex submanifold, we can choose $E_{f}=E_{f^{\prime}}$ as $\mathbb{C}$ vector spaces. We lose the weakly submersion of $\mathcal{M}(X, L) \rightarrow T L$ in the direction of $T C$ but we can have the same perturbed multi-sections. Notice that they have same automorphisms.

Notice that the Calabi-Yau condition of the total space implies the bundle pair $\left(f^{*} N_{X_{t} / X},(\partial f)^{*} N_{L_{t} / L}\right)$ is trivial and thus

$$
H^{0}\left(f^{*} N_{X_{t} / X}, f^{*} N_{L_{t} / L}\right) \cong \mathbb{R}
$$

Moreover, it admits a natural orientation coming from the orientation of $L$ and $L_{t}$. Assume $V$ is an generator of $H^{0}\left(f^{*} N_{X_{t} / X}, f^{*} N_{L_{t} / L}\right)$ and $\tilde{V}$ is the unique lifting to $W^{1, p}\left(f^{*} T X, f^{*} T L\right)$ with smallest $L^{2}$-norm, then by diagram chasing, we have

$$
D_{f} \bar{\partial} \tilde{V} \in L^{p}\left(f^{\prime *} X_{t} \otimes \Lambda^{0,1}\right) \backslash \operatorname{Im} D_{f^{\prime}} \bar{\partial} .
$$


Similar to the construction in section 5 , we consider the new linearized Cauchy-Riemann equation

$$
\begin{aligned}
& D \bar{\partial}_{\text {new }}: W^{1, p}\left(f^{\prime *} T X_{t}, f^{\prime *} T L_{t}\right) \times \mathbb{R}_{\vartheta} \rightarrow L^{p}\left(f^{\prime *} X_{t} \otimes \Lambda^{0,1}\right) \\
&(w, \vartheta) \longmapsto D_{f^{\prime}} \bar{\partial} w+\vartheta D_{f} \bar{\partial} \tilde{V}
\end{aligned}
$$

and one can construct a reduced Kuranishi structure on $\mathcal{M}_{\beta}\left(X_{t}, L_{t}\right)$ depending on the K3 fibration $X \rightarrow \mathbb{P}^{1}$ similar to the proof of Theorem 5.17.

We may choose product metric around a tubular neighborhood of the pair $\left(X_{t}, L_{t}\right)$, then $X_{t} \subseteq X$ is totally geodesic.

Lemma 7.5. The bounded inverses for linearized Cauchy-Riemann operator $D_{f} \bar{\partial}$ and $D_{f^{\prime}} \bar{\partial}$ can be chosen compatible in the sense that the following diagram commute

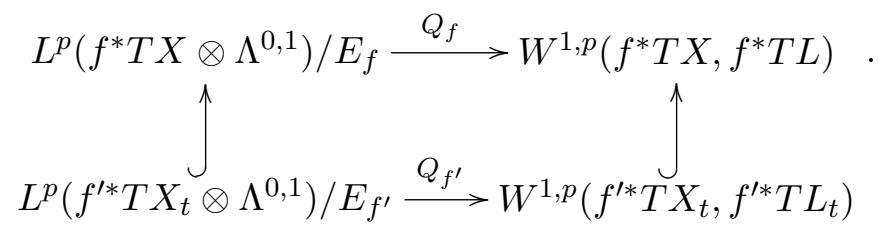

Therefore, when we run the Newton's iteration constructing the Kuranishi map we can make it compatible. To sum up, with very special choices, the Kuranishi structure on $\mathcal{M}_{0, \gamma}\left(X_{t}, L_{t}\right)$ and $\mathcal{M}_{0, \gamma}^{t}(X, L)$ can be chosen exactly the same. Therefore, we have the following proposition of comparing reduced open discs counting invariants on K3 surfaces (depending on the K3 fibration) and open discs counting on Calabi-Yau with K3 fibration.

Proposition 7.6. Let $\gamma \in H_{2}\left(X_{t}, L_{t}\right) \subseteq H_{2}(X, L)$ be a fibral relative class, 
we have

$$
G W_{\gamma}^{\leq \lambda}(X, L)=\sum_{t: \phi(t) \in N L_{\gamma}} G W_{r e d}^{X, \gamma}\left(X_{t}, L_{t}\right)
$$

where

$$
G W_{r e d}^{X, \gamma}\left(X_{t}, L_{t}\right)=\operatorname{Corr}_{*}\left(\mathcal{M}_{0, \gamma}\left(X_{t}, L_{t}\right) ; \text { tri,tri }\right)(1)
$$

Next we want to compare the disc counting invariant defined in this paper using the K3 fibration and the invariant defined in section 5 using twistor space. We first have the following observation

Lemma 7.7. The image of the map in the above diagram

$$
H^{0}\left(D^{2}, \partial D^{2} ; f^{*} N_{X_{t} / X}, f^{*} N_{L_{t} / L}\right) \rightarrow H^{1}\left(D^{2}, \partial D^{2} ; f^{\prime *} X_{t}, f^{\prime *} L_{t}\right)
$$

only depends on the extended Kodaira-Spencer class

$$
d \phi_{\left(X_{t}, L_{t}\right)} \in T_{\left(X_{t}, L_{t}\right)} \mathcal{M}=H^{1}\left(X_{t}, T X_{t}\right) \times \mathcal{H}^{1}\left(L_{t}\right)
$$

but not depends on the actual K3 fibration $X$.

Assume there are two K3 fibration $X$ and $X^{\prime}$ both contains a fibre biholomorphic to $X_{t}$. Let $L$ (and $L^{\prime}$ ) be totally real submanifolds in $X$ (and $X^{\prime}$ ) such that $L \cap X_{t}=L_{t}\left(\right.$ and $\left.L^{\prime} \cap X_{t}=L_{t}\right)$. Let $V \in H^{0}\left(f^{*} N_{X_{t} / X}, f^{*} N_{L_{t} / L}\right)$ and $V^{\prime} \in H^{0}\left(f^{*} N_{X_{t} / X^{\prime}}, N_{L_{t}, L^{\prime}}\right)$ be the generator respectively. By replacing $V$ by $t V+(1-t) V^{\prime}$, one can construct a cobordism of Kuranishi structures using two different $\mathrm{K} 3$ fibration. Thus, the invariant $\left.G W_{r e d}^{\gamma} X^{(} X_{t}, L_{t}\right)$ now 
only depends on the extended Kodaira-Spencer class but not the actual K3 fibration.

Remark 7.8. Notice that in the construction of reduced Kuranishi structure we use $K_{J}(f, \alpha)$, which is exactly gives the pull-back of Kodaira-Spencer class of the twistor line to the disc and zero in the second factor in the extended Kodaira-Spencer class.

Proof. From the the expression (28)

$$
\frac{d J_{t \alpha}}{d t}=\left(\frac{1-t^{2}}{1+t^{2}}\right)^{\prime} J_{\alpha}-\left(\frac{2 t}{1+t^{2}}\right)^{\prime} K_{\alpha},
$$

we know $K_{\alpha}$ is proportional to the projection of $\frac{d J_{t \alpha}}{d t}$ to $(0,1)$-part and thus is a representative of Kodaira-Spencer class induced by the twistor line.

Next we want to show that the invariant is even independent of the generalized Kodaira-Spencer class (up to a sign). Indeed, given a K3 fibration $X$ and above data, one can perturb $L$ (actually perturb $\phi$ ) to achieve any second factor (up to a sign)of the extended Kodaira-Spencer class such that the moduli space of holomorphic discs with totally real boundary condition has no boundary. Here the sign is given by $N L_{\gamma}^{\phi, t}$. By Theorem 6.1 [8], Remark 7.8 and Corollary 5.48, we have the following conclusion:

Lemma 7.9. With the above notation, the two invariants coincide up to sign, namely,

$$
G W_{r e d}^{X, \gamma}\left(X_{t}, L_{t}\right)=N L_{\gamma}^{\phi, t} \cdot \tilde{\Omega}^{X_{t}}\left(\gamma, L_{t}\right)
$$


Together with Proposition 7.6, we finally connect the genus zero reduced open Gromov-Witten on K3 surfaces and genus zero open Gromov-Witten invariants on Calabi-Yau 3-folds with K3 fibrations:

Theorem 7.10. Let $X$ is a Calabi-Yau 3-folds with K3 fibration. Let $\phi$ : $S^{1} \rightarrow \mathcal{M}_{X}$ be an immersion such that

1. $\phi\left(S^{1}\right)$ avoids the walls of marginal stability of energy less than $\lambda$

2. $\phi\left(S^{1}\right)$ intersect $N L_{\gamma}$ only finitely many times, for $\gamma$ with energy less than $\lambda$.

then we have the the formula

$$
G W_{\gamma}^{\leq \lambda}(X, L)=\sum_{t: \phi(t) \in N L_{\gamma}} N L_{\gamma}^{\phi, t} \cdot \tilde{\Omega}^{X_{t}}\left(\gamma, L_{t}\right)
$$

Notice that here we don't have to assume that $\phi\left(S^{1}\right)$ intersect $N L_{\gamma}$ transversally.

As a corollary of Theorem 7.10, the integrality conjecture 5.58 for the reduced open Gromov-Witten invariants on K3 surfaces implies the integrality Conjecture 8.2 [8] of open Gromov-Witten invariants on Calabi-Yau 3 -folds.

Remark 7.11. Professor Vafa pointed out that similar work done for flat $\mathbb{C}^{2}$ in $[5]$. 


\section{A Miscellaneous of Kuranishi Structures}

This Appendix is a review of standard things of Kuranishi spaces we need in this article. Except Proposition A.16 doesn't appears in existing literature, all the rest can be found in [7][12][13] with more details.

\section{A.1 Kuranishi Structure and Good Coordinate}

Definition A.1. (Kuranishi neighborhood) Let $M$ be a Haudorff topological space. A Kuranishi neighborhood of $p \in M$ is a 5-tuple $\left(V_{p}, E_{p}, \Gamma_{p}, \psi_{p}, s_{p}\right)$ such that

1. $V_{p}$ is a smooth manifold (with corners) and $E_{p}$ is a smooth vector bundle over $V_{p}$.

2. $\Gamma_{p}$ is a finite group acting on $E_{p} \rightarrow V_{p}$.

3. $s_{p}$ is a $\Gamma_{p}$-invariant continuous section of $E_{p}$.

4. $\psi_{p}: s^{-1}(0) \rightarrow M$ is continuous and induced homeomorphism between $s^{-1}(0) / \Gamma_{p}$ and a neighborhood of $p \in M$.

For $x \in V_{p}$, we denote the isotropy subgroup at $x$ by

$$
\left(\Gamma_{p}\right)_{x}=\left\{\gamma \in \Gamma_{p} \mid \gamma x=x\right\}
$$

Let $o_{p} \in V_{p}$ such that $s\left(o_{p}\right)=0$ and $\psi_{p}\left(\left[o_{p}\right]\right)=p$. We will assume $o_{p}$ is fixed by all elements of $\Gamma_{p}$ and thus the unique point of $V_{p}$ mapping to $p$ via $\psi_{p}$.

Definition A.2. (Kuranishi structure) A Kuranishi structure on a Hausdorff topological space $M$ is an assignment of Kuranishi neighborhood 
$\left(V_{p}, E_{p}, \Gamma_{p}, s_{p}, \psi_{p}\right)$ for each $p \in M$ and a 4-tuple $\left(V_{p q}, \hat{\phi}_{p q}, \phi_{p q}, h_{p q}\right)$ to each pair $(p, q)$ where $p \in M$ and $q \in \psi_{p}\left(s_{p}^{-1}(0) / \Gamma_{p}\right)$ satisfying:

1. $V_{p q}$ is an open subset of $V_{q}$ containing $o_{q}$.

2. $h_{p q}$ is an injective homomorphism from $\Gamma_{q}$ to $\Gamma_{p}$ such that restricts to an isomorphism $\left(\Gamma_{q}\right)_{x} \rightarrow\left(\Gamma_{p}\right)_{\phi_{p q}(x)}$ for any $x \in V_{p q}$

3. $\phi_{p q}: V_{p q} \rightarrow V_{p}$ is an $h_{p q}$-equivariant embedding such that it descends to injective $\operatorname{map} \underline{\phi}_{p q}: U_{p q}=V_{p q} / \Gamma_{p} \rightarrow U_{q}=V_{q} / \Gamma_{q}$.

4. $\hat{\phi}_{p q}:\left.E\right|_{V_{p q}} \rightarrow E_{p}$ is an $h_{p q}$-equivariant embedding of vector bundles covering $\phi_{p q}$.

5. $\hat{\phi}_{p q} \circ s_{q}=s_{p} \circ \phi_{p q}$

6. $\psi_{q}=\psi_{p} \circ \underline{\phi}_{p q}$ on $\left(s_{q}^{-1}(0) \cap V_{p q}\right) / \Gamma_{q}$.

7. If $r \in \psi_{q}\left(s_{q}^{-1} \cap V_{p q}\right)$, then $\hat{\phi}_{p q} \circ \hat{\phi}_{q r}=\hat{\phi}_{p r}$ in a neighborhood of $\psi^{-1}(0)$.

8. The virtual dimension $\operatorname{dim} V_{p}-\operatorname{dim} E_{p}$ of the Kuranishi structure is independent of $p$.

9. (Cocycle condition) If $r \in \psi_{q}\left(\left(V_{p q} \cap s_{q}^{-1}(0)\right) / \Gamma_{p}\right), q \in \psi_{p}\left(s_{p}^{-1}(0) / \Gamma_{p}\right)$, then there exists $\gamma_{p q r}^{\alpha} \in \Gamma_{p}$ for each connected component $\left(\phi_{q r}^{-1}\left(V_{p q}\right) \cap\right.$ $\left.V_{q r} \cap V_{p r}\right)_{\alpha}$ of $\phi_{q r}^{-1}\left(V_{p q}\right) \cap V_{q r} \cap V_{p r}$ such that

$$
\begin{array}{r}
h_{p q} \circ h_{q r}=\gamma_{p q r}^{\alpha} \cdot h_{p r} \cdot\left(\gamma_{p q r}^{\alpha}\right)^{-1}, \\
\phi_{p q} \circ \phi_{q r}=\gamma_{p q r}^{\alpha} \cdot \phi_{p r}, \quad \hat{\phi}_{p q} \circ \hat{\phi}_{q r}=\gamma_{p q r}^{\alpha} \cdot \hat{\phi}_{p r} .
\end{array}
$$


We call a Hausdorff topological space equipped with Kuranishi structure a Kuranishi space.

Moreover, we will ask $\left\{V_{\alpha}, E_{\alpha}, \Gamma_{\alpha}, s_{\alpha}, \psi_{\alpha} \mid \alpha \in \mathfrak{U}\right\}$ is a good coordinate system in the following sense: We have a partial order $<$ on $\mathfrak{U}$, such that $\alpha_{1} \leq \alpha_{2}$ or $\alpha_{2} \leq \alpha_{1}$ for $\alpha_{1}, \alpha_{2} \in \mathfrak{U}$ if

$$
\psi_{\alpha_{1}}\left(s_{\alpha_{1}}^{-1}(0) / \Gamma_{\alpha_{1}}\right) \cap \psi_{\alpha_{2}}\left(s_{\alpha_{2}}^{-1}(0) / \Gamma_{\alpha_{2}}\right) \neq \varnothing
$$

Assume that $\alpha_{1}<\alpha_{2}$, then we have an injective homomorphism $h_{\alpha_{1} \alpha_{2}}$ : $\Gamma_{\alpha_{1}} \rightarrow \Gamma_{\alpha_{2}}, \Gamma_{\alpha_{2}}$-invariant open set $V_{\alpha_{1} \alpha_{2}} \subseteq V_{\alpha_{1}}$ such that there is an $h_{\alpha_{1} \alpha_{2}}$-equivariant embedding of open set $\phi_{\alpha_{1} \alpha_{2}}: V_{\alpha_{1} \alpha_{2}} \rightarrow V_{\alpha_{2}}$, and $h_{\alpha_{1} \alpha_{2}}{ }$ equivariant bundle map $\hat{\phi}_{\alpha_{1} \alpha_{2}}:\left.E_{\alpha_{1}}\right|_{V_{\alpha_{1} \alpha_{2}}} \rightarrow E_{\alpha_{2}}$ cover $\phi_{\alpha_{1} \alpha_{2}}$ such that analogue of above 5,6,7 are satisfied (more details see [13]).

Remark A.3. [13] section 7 guarantees the existence of good coordinate for any compact Kuranishi space.

Assume $X_{i}=\left(V^{i}, E^{i}, \Gamma^{i}, \psi^{i}, s^{i}\right)$ Kuranishi structure and $f_{i}: X_{i} \rightarrow Y_{i}$ strongly continuous and weakly submersive. Let $Y=\prod Y_{i}, W$ some manifold with corners and $f: W \rightarrow Y$ a smooth map. We can construct a Kuranishi structure on

$$
Z=\prod_{i} X_{i} \times_{Y} W
$$

by taking

$$
V_{i} \prod_{i} V^{i} \times_{Y} W, \quad E=\prod_{i} E^{i}, \quad \Gamma=\prod_{i} \Gamma^{i}
$$


Since $f_{i}$ are submersions, $V$ is a smooth manifold. It is easy to define $s$ and $\psi$ in a natural way.

Definition A.4. (Fibre product Kuranishi structure) Let $X_{i}$ have Kuranishi structures. Let $f_{i}: X_{i} \rightarrow Y$ to be strongly continuous and weakly submersive. We define the Kurnishi structure on $Z=X_{1} \times_{Y} X_{2}$ by identify $Z=\left(X_{1} \times\right.$ $\left.X_{2}\right) \times_{Y^{2}} Y$, where $Y \rightarrow Y^{2}, y \rightarrow(y, y)$.

\section{A.2 Partition of Unity}

Fix $\epsilon>0$ sufficiently small and $\chi^{\delta}: \mathbb{R} \rightarrow[0,1]$ smooth function such that

$$
\chi^{\epsilon}(s)= \begin{cases}0, & \text { if } s>\epsilon \\ 1, & \text { if } s<\frac{\epsilon}{2}\end{cases}
$$

For each $x \in V_{\alpha}$, we put

$$
\begin{aligned}
& \mathfrak{U}_{x,+}=\left\{\alpha_{+} \mid x \in V_{\alpha \alpha_{+}}, \alpha<\alpha_{+}\right\} \\
& \mathfrak{U}_{x,-}=\left\{\alpha_{-} \mid\left[x \bmod \Gamma_{\alpha}\right] \in U_{\epsilon}\left(V_{\alpha_{-} \alpha} / \Gamma_{\alpha_{-}}\right), \alpha_{-}<\alpha\right\} .
\end{aligned}
$$

For $\alpha_{-} \in \mathfrak{U}_{x,-}$, we take $x_{\alpha_{-}} \in N_{V_{\alpha_{-}}} V_{\alpha}$ such that $\operatorname{Exp}\left(x_{\alpha_{-}}\right)=x$.

Definition A.5. A system $\left\{\chi_{\alpha} \mid \alpha \in \mathfrak{U}\right\}$ of $\Gamma_{\alpha}$-equivariant smooth functions $\chi_{\alpha}: V_{\alpha} \rightarrow[0,1]$ with compact support is a partition of unity subordinate to 
the given good (Kuranishi) coordinate if for each $x \in V_{\alpha}$,

$$
\chi_{\alpha}(x)+\sum_{\alpha_{-} \in \mathfrak{U}_{x,-}} \chi^{\epsilon}\left(\left\|x_{\alpha_{-}}\right\|\right) \chi_{\alpha_{-}}\left(P r_{\alpha_{-} \alpha}\left(x_{\alpha_{-}}\right)\right)+\sum_{\alpha_{+} \in \mathfrak{U}_{x,+}} \chi_{\alpha_{+}}\left(\phi_{\alpha \alpha_{+}}(x)\right)=1
$$

\section{A.3 Multi-Sections and Compatible Perturbations}

Let $\left(V_{\alpha}, E_{\alpha}, \Gamma_{\alpha}, s_{\alpha}, \psi_{\alpha}\right)$ be a Kuranihis chart of $\mathcal{M}$ and $x \in V_{\alpha}$. Set $\mathcal{S}^{l}\left(E_{\alpha, x}\right)$ be the $l$-fold symmetric product of $E_{\alpha, x}$. There is a natural map

$$
\begin{aligned}
t m_{m}: \mathcal{S}^{l}\left(E_{\alpha, x}\right) & \rightarrow \mathcal{S}^{l m}\left(E_{\alpha, x}\right) \\
{\left[a_{1}, \ldots, a_{l}\right] } & \mapsto \underbrace{a_{1}, \ldots, a_{1}}_{m \text { copies }}, \ldots, \underbrace{a_{l}, \ldots, a_{l}}_{m \text { copies }}]
\end{aligned}
$$

A smooth multi-section $s$ of the orbibundle $E_{\alpha} \rightarrow V_{\alpha}$ is a set of data $s_{i}$, such that $s_{i}(x) \in \mathcal{S}^{l_{i}}\left(E_{\alpha, x}\right)$ for an open cover $\left\{U_{\alpha, i}\right\}$ of $V_{\alpha}$ satisfying

1. $U_{\alpha, i}$ are $\Gamma_{\alpha}$-invariant and $s_{i}$ is $\Gamma_{\alpha}$-equivariant.

2. If $x \in U_{\alpha, i} \cap U_{\alpha, j}$, then

$$
t m_{l_{j}}\left(s_{i}(x)\right)=t m_{l_{i}}\left(s_{j}(x)\right) \in \mathcal{S}^{l_{i} l_{j}}\left(E_{\alpha, \gamma x}\right)
$$

3. There exists local smooth lifting $\tilde{s}$.

We identify two multi-section $\left(\left\{U_{i}\right\},\left\{s_{i}\right\},\left\{l_{i}\right\}\right),\left(\left\{U_{i}^{\prime}\right\},\left\{s_{i}^{\prime}\right\},\left\{l_{i}^{\prime}\right\}\right)$ if

$$
t m_{l_{j}}\left(s_{i}(x)\right)=t m_{l_{i}}\left(s_{j}^{\prime}(x)\right) \in \mathcal{S}^{l_{i} l_{j}^{\prime}}\left(E_{\alpha, \gamma x}\right) \quad \text { on } U_{i} \cap U_{j}^{\prime} \text {. }
$$


To add up two multi-sections $s^{(1)}, s^{(2)}$ together, we first refine the associated open cover if necessarily such that they coincides and same automorphism on each open cover. Then we can define

$$
\begin{aligned}
+: \quad \mathcal{S}^{l_{1}}(E) \times \mathcal{S}^{l_{2}}(E) \longrightarrow \mathcal{S}^{l_{1} l_{2}}(E) \\
\quad\left(\left[a_{1}, \cdots, a_{l_{1}}\right],\left[b_{1}, \cdots, b_{l_{2}}\right]\right) \mapsto\left[a_{i}+b_{j}: i=1, \cdots, l_{1}, b=1, \cdots, l_{2}\right]
\end{aligned}
$$

It is easy to check that + is well-defined, associative and commutative. However, it only has monoid structure. Another thing worth notice is although $C^{0}(\mathcal{M})$ acts on the sets of multi-sections, we don't have $(f+g) s=f s+g s$, for $f, g \in C^{0}(\mathcal{M})$ !

To make the integration along fibre well-defined, we introduce the auxiliary manifold $W_{\alpha}$ which is a finite dimensional smooth oriented manifold. We consider the the pull-back bundle

$$
\pi^{*} E_{\alpha} \rightarrow W_{\alpha} \times V_{\alpha}
$$

and the action of $\Gamma_{\alpha}$ acts on $W_{\alpha}$ is trivial.

Definition A.6. (perturbed multi-section)

1. A $W_{\alpha}$-parametrized family $\mathfrak{s}_{\alpha}$ of multi-section $s_{\alpha}$ is a multi-section of $\pi^{*} E_{\alpha}$.

2. Fix a metric on the bundle $E$. We say $\mathfrak{s}_{\alpha}$ is $\epsilon$-closed to $s$ if each branch $\mathfrak{s}_{\alpha, i, j}$, we have

$$
\left|\mathfrak{s}_{\alpha, i, j}(w, \cdots)-s_{\alpha}(\cdots)\right|_{C^{0}}<\epsilon
$$


in a neighborhood of $x$, for each $(w, x) \in W_{\alpha} \times V_{\alpha}$.

3. $\mathfrak{s}_{\alpha}$ is transverse to 0 if every branch $\mathfrak{s}_{\alpha, i, j}$ is transverse to 0 .

4. With above properties, $\left.f_{\alpha}\right|_{\mathfrak{s}_{\alpha}^{-1}(0)}$ is a submersion if restriction to zero locus of each branch is a submersion.

Lemma A.7. Assume $f_{\alpha}: V_{\alpha} \rightarrow M$ is a submersion, then there exists $W_{\alpha}$ such that for any $\epsilon$ there exists a $W_{\alpha}$-parametrized family of multi-sections $\mathfrak{s}_{\alpha}$ satisfying:

1. $\mathfrak{s}_{\alpha}$ is $\epsilon$-closed to $s_{\alpha}$.

2. $\mathfrak{s}_{\alpha}$ is transverse to zero section.

3. $\left.f\right|_{\mathfrak{s}_{\alpha}^{-1}(0)}$ is a submersion.

4. $\mathfrak{s}_{\alpha}(v, 0)=s_{\alpha}(0)$.

Moreover, if a given $\mathfrak{s}_{\alpha}$ satisfies the condition on a neighborhood of $\Gamma_{\alpha^{-}}$ invariant compact subset in $V$, then we may extend it to $V_{\alpha}$.

Proof. We first choose $W_{\alpha}$ to be a vector space with dimension large enough such that

$$
\operatorname{Sur}_{\alpha}: W_{\alpha} \times V_{\alpha} \rightarrow E_{\alpha}
$$

is a surjective bundle map (not necessarily $\Gamma_{\alpha}$-equivariant). Set

$$
\mathfrak{s}_{\alpha}^{(1)}(w, x)=\operatorname{Sur}_{\alpha}(w, x)+s_{\alpha}(x)
$$


and

$$
\mathfrak{s}_{\alpha}^{(2)}(w, x)=\left[\gamma_{1} \mathfrak{s}_{\alpha}^{(1)}(w, x), \cdots, \gamma_{g} \mathfrak{s}_{\alpha}^{(1)}(w, x)\right]
$$

where $\Gamma_{\alpha}=\left\{\gamma_{1}, \cdots, \gamma_{g}\right\}$. $\mathfrak{s}_{\alpha}^{(2)}$ defines a multisection on $W_{\alpha} \times V_{\alpha}$ which is transverse to 0 because the extra dimension from the auxiliary $W_{\alpha}$. Finally, $\left(\mathfrak{s}_{\alpha}^{(2)}\right)^{-1}(0) \rightarrow V_{\alpha}$ is submersive implies that $\left.f\right|_{\left(\mathfrak{s}_{\alpha}^{(2)}\right)^{-1}(0)}$ is a submersion.

Theorem A.8. [12] There exists a system of multi-sections $\mathfrak{s}_{k+1, \beta}$ on $\mathcal{M}_{k+1, \beta}(X, L)$ such that

1. They are transverse to 0 .

2. ev $v_{0}$ induces submersion on the zero sets of $\mathfrak{s}_{k+1, \beta}$.

3. The multi-section is preserved by cyclic permutations of boundary points.

4. The multi-section $\mathfrak{s}_{k+1, \beta}$ is the pull-back of the multi-section $\mathfrak{s}_{k, \beta}$ by the forgetful map.

5. The restriction of multi-section to the boundary is compatible.

Remark A.9. We don't need the geometry of the moduli space to construct perturbed multi-sections but need the Kuranishi structure itself.

Remark A.10. We can take ev $v_{0}$ to be weakly submersive and by cyclic symmetry each ev $v_{i}$ is weakly submersive. However, if we ask the choice of multi-section is compatible with the forgetful map then the map $\left(e v_{1}, \cdots, e v_{k}\right)$ can not be weakly submersive anymore by trivial dimensional counting argument. However, ev $v_{0}$ is weakly submersive already can pullback differential forms and define the de Rham model. 
Moreover, we want the family of multi-section $\mathfrak{s}_{k, \beta}$ satisfies the compatibility with respect to the good coordinate. Let $\alpha_{1}<\alpha_{2}$ : Choose an $\Gamma_{\alpha_{2}}$-invariant metric on $V_{\alpha_{2}}$ and consider the exponential map

$$
\operatorname{Exp}_{\alpha_{1} \alpha_{2}}: B_{\epsilon} N_{\alpha_{1} \alpha_{2}} V_{\alpha_{2}} \rightarrow V_{\alpha_{2}}
$$

and denotes the image by $U_{\epsilon}\left(V_{\alpha_{1} \alpha_{2}} / \Gamma_{\alpha_{1}}\right) \subseteq V_{\alpha_{2}} / \Gamma_{\alpha_{2}}$. We extend the orbibundle $E_{\alpha_{1}}$ to $U_{\epsilon}\left(V_{\alpha_{1} \alpha_{2}} / \Gamma_{\alpha_{1}}\right)$ by pullback of projection

$$
\operatorname{Pr}_{V_{\alpha_{1} \alpha_{2}}}: U_{\epsilon}\left(V_{\alpha_{1} \alpha_{2}} / \Gamma_{1}\right) \rightarrow V_{\alpha_{1} \alpha_{2}} / \Gamma_{\alpha_{1}}
$$

and also extend the embedding $E_{\alpha_{1}} \rightarrow \hat{\phi}_{\alpha_{1} \alpha_{2}}^{*} E_{\alpha_{2}} E_{\alpha_{2}}$ to $U_{\epsilon}\left(V_{\alpha_{1} \alpha_{2}} / \Gamma_{\alpha_{1}}\right)$. Fix a $\Gamma_{\alpha}$-invariant invariant inner product on $E_{\alpha}$ and we have a bundle isomorphism

$$
E_{\alpha_{2}} \cong E_{\alpha_{1}} \oplus \frac{\hat{\phi}_{\alpha_{1} \alpha_{2}}^{*} E_{\alpha_{2}}}{E_{\alpha_{1}}}
$$

over $U_{\epsilon}\left(V_{\alpha_{1} \alpha_{2}} / \Gamma_{1}\right)$. One might need to use implicit function theorem and tangent bundle to modify $\operatorname{Pr}_{\alpha_{1} \alpha_{2}}$ such that

$$
d s_{\alpha_{2}}\left(\tilde{y} \bmod T V_{\alpha_{1}}\right) \equiv s_{\alpha_{2}}(y) \bmod E_{\alpha_{1}}
$$

if $y=\operatorname{Exp}_{\alpha_{1} \alpha_{2}}(\tilde{y}) \in U_{\epsilon}\left(V_{\alpha_{1} \alpha_{2}} / \Gamma_{\alpha_{1}}\right)$.

Definition A.11. If $\alpha_{1}<\alpha_{2}$, then $\mathfrak{s}_{\alpha_{2}}$ is compatible with $\mathfrak{s}_{\alpha_{1}}$ if for each 
$y=\operatorname{Exp}_{\alpha_{1}, \alpha_{2}}(\tilde{y}) \in U_{\epsilon}\left(V_{\alpha_{1} \alpha_{2}} / \Gamma_{\alpha_{1}}\right)$, we have

$$
\mathfrak{s}_{\alpha_{2}}(\tilde{y})=\mathfrak{s}_{\alpha_{1}}(\operatorname{Pr}(\tilde{y})) \oplus d s_{\alpha_{2}}\left(\tilde{y} \bmod T V_{\alpha_{1}}\right) .
$$

via isomorphism $E_{\alpha_{2}} \cong E_{\alpha_{1}} \oplus \frac{\phi_{\alpha_{1} \alpha_{2}}^{*} T V_{\alpha_{2}}}{T V_{\alpha_{1}}}$ assuming the moduli space is oriented.

\section{A.4 Smooth Correspondence}

Let $\mathcal{M}$ with Kuranishi structure $\left(V_{\alpha}, E_{\alpha}, \Gamma_{\alpha}, s_{\alpha}, \psi_{\alpha}\right), f^{s}: \mathcal{M} \rightarrow N_{s}$ strongly continuous and $f^{t}: \mathcal{M} \rightarrow N_{t}$ strongly continuous and weakly submersive. Here we assume $N_{s}, N_{t}$ are both smooth manifolds (might with boundaries or corners). We will define smooth correspondence

$$
\operatorname{Corr}_{*}\left(\mathcal{M} ; f^{s}, f^{t}\right): \Lambda^{d}\left(N_{s}\right) \rightarrow \Lambda^{d+\operatorname{dim} N_{t}-\operatorname{dim} N_{s}}\left(N_{t}\right)
$$

We first take a compatible continuous family of multi-sections $\mathfrak{s}_{\alpha}=\left\{\mathfrak{s}_{\alpha, i, j} \mid j=\right.$ $\left.1, \cdots l_{i}\right\}$ satisfies the lemma and $\tilde{\mathfrak{s}}_{\alpha, i, j}$ is the local smooth lifting. Let $\rho \in$ $\Lambda\left(N_{s}\right)$. Consider a branch $\tilde{\mathfrak{s}}_{\alpha, i, j}$ as a section of $E_{\alpha}$ over $U_{\alpha, i} \times W_{\alpha}$. We choose a volume form $\omega_{\alpha}$ on $W_{\alpha}$ with total mass 1 and support on an $\epsilon$ neighborhood of $0 \in W_{\alpha}$ and partition of unity $\chi_{i}$ for open cover $\left\{U_{\alpha, i}\right\}_{i}$. Then

$$
\left.\frac{1}{\# \Gamma_{\alpha}} \sum_{i} \sum_{j=1}^{l_{i}}\left(\left.f_{\alpha}^{t} \circ \pi_{\alpha}\right|_{\tilde{\mathfrak{s}}_{\alpha, i, j}^{-1}(0)}\right) ! \frac{1}{l_{i}}\left(\chi_{i} \chi_{\alpha}\left(f_{\alpha}^{s}\right)^{*} \rho \wedge \omega_{\alpha}\right)\right|_{\tilde{\mathfrak{s}}_{\alpha, i, j}^{-1}(0)}
$$

defines the $U_{\alpha, i}$ part of the smooth correspondence $\operatorname{Corr}_{*}\left(\mathcal{M} ; f^{s}, f^{t}\right)(\rho)$ and we use partition unity $\chi_{\alpha}$ for summing various $\chi_{\alpha}$ to glue them together. 
Remark A.12. The definition of smooth correspondence $\operatorname{Corr}_{*}\left(\mathcal{M} ; f^{s}, f^{t}\right)$ only depends on the Kuranishi structure $\left(V_{\alpha}, E_{\alpha}, \Gamma_{\alpha}, s_{\alpha}, \psi_{\alpha}\right)$, the auxiliary $\left(W_{\alpha}, \omega_{\alpha}\right)$, the perturbation $\mathfrak{s}_{\alpha}, f_{\alpha}$ but not depends on other choices.

Remark A.13. Although one may not be able to exclude the case $\mathcal{M}_{\beta}$ has infinitely many components. However, $\operatorname{Corr}_{*}\left(\mathcal{M}_{\beta} ;\right.$ tri,tri $)$ is always finite.

Proposition A.14. (Stoke's theorem)

$$
d \circ \operatorname{Corr}_{*}\left(\mathcal{M} ; f^{s}, f^{t}\right)-\operatorname{Corr}_{*}\left(\mathcal{M} ; f^{s}, f^{t}\right) \circ d=\operatorname{Corr}_{*}\left(\partial \mathcal{M} ; f^{s}, f^{t}\right)
$$

Proposition A.15. (composition formula) Let $\rho_{i} \in \Lambda\left(N_{s}^{i}\right), i=1,2$ with the following diagram,

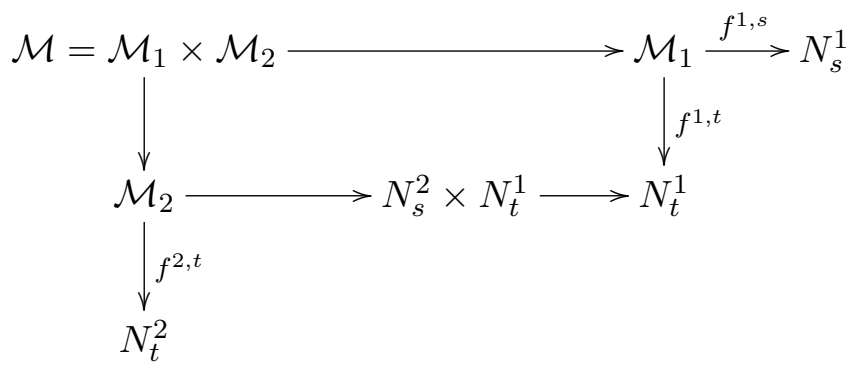

then we have

$$
\begin{aligned}
& \operatorname{Corr}_{*}\left(\mathcal{M} ; f^{s}, f^{t}\right)\left(\rho_{1} \times \rho_{2}\right) \\
= & \operatorname{Corr}_{*}\left(\mathcal{M}_{2} ; f^{2, s}, f^{2, t}\right)\left(\operatorname{Corr}_{*}\left(\mathcal{M}_{1} ; f^{1, s}, f^{1, t}\right)\left(\rho_{1}\right) \times \rho_{2}\right)
\end{aligned}
$$

At the end, we have an open analogue of divisor axiom though we never use it. 
Proposition A.16. Assume $\beta$ is primitive and $L$ doesn't fall on $W_{\beta}^{\prime}$. Let $\rho \in \Lambda^{1}\left(L \times S_{\vartheta}^{1}\right), d \rho=0$, and forget $: \mathcal{M}_{1, \beta}(\mathfrak{X}, L) \rightarrow \mathcal{M}_{0, \beta}(\mathfrak{X}, L)$, then

$$
\operatorname{Corr}_{*}\left(\mathcal{M}_{1, \beta}(L) ;\left(e v_{0}, e v_{\vartheta}\right), \text { forget }\right)(\rho)=\left(\int_{\partial \beta} \rho\right) \cdot m_{-1, \beta}(L)
$$

Proof.

$$
\begin{aligned}
& \int_{L \times S_{\vartheta}^{1}} m_{0, \beta} \wedge \rho \\
= & \int_{L \times S_{\vartheta}^{1}} \operatorname{Corr}_{*}\left(\mathcal{M}_{1, \beta}(L) ; \operatorname{tri},\left(e v_{0}, e v_{\vartheta}\right)\right)(1) \wedge \rho \\
= & \operatorname{Corr}_{*}\left(\mathcal{M}_{1, \beta}(L) ;\left(e v_{0}, e v_{\vartheta}\right), \operatorname{tri}\right)(\rho) \\
= & \operatorname{Corr}_{*}\left(\mathcal{M}_{1, \beta}(L) ;\left(e v_{0}, e v_{\vartheta}\right), \operatorname{tri} \circ \text { forget }\right)(\rho) \\
= & \operatorname{Corr}_{*}\left(\mathcal{M}_{0, \beta}(L) ; i d, \operatorname{tri}\right)\left(\operatorname{Corr}_{*}\left(\mathcal{M}_{1, \beta}\left(L_{t}\right) ;\left(e v_{0}, e v_{\vartheta}\right), \mathfrak{f o r g} \mathfrak{g t}\right)(\rho)\right) \\
= & \operatorname{Corr}_{*}\left(\mathcal{M}_{0, \beta}(L) ; \operatorname{tri}, \operatorname{tri}\right) \cdot \int_{\partial \beta} \rho \\
= & m_{-1, \beta} \cdot \int_{\partial \beta} \rho
\end{aligned}
$$

The second equality is from the projection formula. The fourth equality is a bit subtle. The smooth correspondence is originally defined only when the target is a smooth manifold. However, the compatibility of forgetful map guarantees the definition extends to this case.

\section{References}

[1] A. Butscher, Deformations of minimal Lagrangian submanifolds with boundary, Proc. Amer. Math. Soc. 131 (2003), no. 6, 1953-1964. 
[2] P. Biran and O. Cornea, Quantum Structures for Lagrangian Submanifolds, preprint, math.SG/0708.4221.

[3] J. Bryan and N. C. Leung, The enumerative geometry of K3 surfaces and modular forms, J. Amer. Math. Soc. 13 (2000), no. 2, 371-410.

[4] K. Chan, The Ooguri-Vafa metric, holomorphic discs and wall-crossing, Math. Res. Lett. 17 (2010), no. 3, 401-414.

[5] Vafa Cumrun and S. Cecotti, BPS wall crossing and topological strings, preprint 2009, hep-th/0910.2615.

[6] F. Denef and G. W. Moore, Split states, entropy enigmas, holes and halos, J. High Energy Phys. (2011), no. 11, 129, i, 152.

[7] K. Fukaya, Cyclic symmetry and adic convergence in Lagrangian Floer theory, Kyoto J. Math. 50 (2010), no. 3, 521-590.

[8] _ Counting pseudo-holomorphic discs in Calabi-Yau 3-folds, Tohoku Math. J. (2) 63, no. 4, 697-727.

[9] _ Multivalued Morse theory, asymptotic analysis and mirror symmetry, Graphs and patterns in mathematics and theoretical physics, 2005, pp. 205-278.

[10] U. Frauenfelder, Gromov convergence of pseudoholomorphic disks, J. Fixed Point Theory Appl. 3 (2008), no. 2, 215-271.

[11] K. Fukaya and K. Ono, Arnold conjecture and Gromov-Witten invariant, Topology 38 (1999), no. 5, 933-1048.

[12] K. Fukaya, Y.-G. Oh, H. Ohta, and K. Ono, Lagrangian intersection Floer theory: anomaly and obstruction. Part II, AMS/IP Studies in Advanced Mathematics, vol. 46, American Mathematical Society, Providence, RI, 2009.

[13] _ Technical details on Kuranishi structure and virtual fundamental chain, preprint 2012, math.SG/1209.4410.

[14] M. Gross, Examples of special Lagrangian fibrations, Symplectic geometry and mirror symmetry (Seoul, 2000), 2001, pp. 81-109.

[15] B. Guan and Q. Li, Complex Monge-Ampère equations and totally real submanifolds, Adv. Math. 225 (2010), no. 3, 1185-1223. 
[16] M. Gross, R. Pandharipande, and B. Siebert, The tropical vertex, Duke Math. J. 153 (2010), no. 2, 297-362.

[17] D. Gaiotto, G. W. Moore, and A. Neitzke, Four-dimensional wall-crossing via threedimensional field theory, Comm. Math. Phys. 299 (2010), no. 1, 163-224.

[18] M. Gross and B. Siebert, From real affine geometry to complex geometry, Ann. of Math. (2) 174 (2011), no. 3, 1301-1428.

[19] M. Gross and P. M. H. Wilson, Large complex structure limits of K3 surfaces, J. Differential Geom. 55 (2000), no. 3, 475-546.

[20] B. R. Greene, A. Shapere, C. Vafa, and S.-T. Yau, Stringy cosmic strings and noncompact Calabi-Yau manifolds, Nuclear Phys. B 337 (1990), no. 1, 1-36.

[21] T. Graber and E. Zaslow, Open-string Gromov-Witten invariants: calculations and a mirror "theorem", Orbifolds in mathematics and physics (Madison, WI, 2001), 2002, pp. $107-121$.

[22] N. J. Hitchin, A. Karlhede, U. Lindström, and M. Roček, Hyper-Kähler metrics and supersymmetry, Comm. Math. Phys. 108 (1987), no. 4, 535-589.

[23] M. Kontsevich and Y. Soibelman, Affine structures and non-Archimedean analytic spaces, The unity of mathematics, 2006, pp. 321-385.

[24] _ Stability structures, motivic Donaldson-Thomas invariants and cluster transformations, preprint 2008, math. AG/ 0811.2435v1.

[25] S. Katz and C.-C. M. Liu, Enumerative geometry of stable maps with Lagrangian boundary conditions and multiple covers of the disc, Adv. Theor. Math. Phys. 5 (2001), no. 1, 1-49.

[26] J. Lee, Family Gromov-Witten invariants for Kähler surfaces, Duke Math. J. 123 (2004), no. 1, 209-233.

[27] P. Lu, Kähler-Einstein metrics on Kummer threefold and special Lagrangian tori, Comm. Anal. Geom. 7 (1999), no. 4, 787-806.

[28] C.-C. Melissa Liu, Moduli of J-Holomorphic curves with Lagrangian Boundary conditions and Open Gromov-Witten Invariants for an $S^{1}$-Equivariant Pair, preprint 2002, math.SG/0210257v2. 
[29] W. Lu, Instanton Correction, Wall Crossing and Mirror Symmetry of Hitchin's Moduli Spaces, to appear on JDG.

[30] Y.-S. Lin, Noether-Lefschetz Theory and Open Gromov-Witten Invariants, in preparation.

[31] H. Ooguri and C. Vafa, Summing up Dirichlet instantons, Phys. Rev. Lett. 77 (1996), no. $16,3296-3298$.

[32] R. Pandharipande and D. Maulik, Gromov-Witten theory and Noether-Lefschetz theory, preprint 2007, math.AG/0705.1653.

[33] R. Pandharipande, J. Solomon, and J. Walcher, Disk enumeration on the quintic 3-fold, J. Amer. Math. Soc. 21 (2008), no. 4, 1169-1209.

[34] M. Reineke, Moduli of representations of quivers, Trends in representation theory of algebras and related topics, 2008, pp. 589-637.

[35] G. Schwarz, Hodge decomposition - a method for solving boundary value problems, Lecture Notes in Mathematics, vol. 1607, Springer-Verlag, Berlin, 1995.

[36] A. Strominger, S.-T. Yau, and E. Zaslow, Mirror symmetry is T-duality, Nuclear Phys. B 479 (1996), no. 1-2, 243-259.

[37] C. Wendl, Automatic transversality and orbifolds of punctured holomorphic curves in dimension four, Comment. Math. Helv. 85 (2010), no. 2, 347-407.

[38] S.-T. Yau, On the Ricci curvature of a compact Kähler manifold and the complex Monge-Ampère equation. I, Comm. Pure Appl. Math. 31 (1978), no. 3, 339-411.

[39] R. Ye, Gromov's compactness theorem for pseudo holomorphic curves, Trans. Amer. Math. Soc. 342 (1994), no. 2, 671-694.

[40] S.-T. Yau and E. Zaslow, BPS states, string duality, and nodal curves on K3, Nuclear Phys. B (1996), no. 3, 503-512. 